PUPT-2395

\title{
The Coset Spin-4 Casimir Operator and Its Three-Point Functions with Scalars
}

\author{
Changhyun Ahn \\ Department of Physics, Kyungpook National University, Taegu 702-701, Korea \\ Department of Physics, Princeton University, Jadwin Hall, Princeton, NJ 08544, USA \\ ahn@knu.ac.kr
}

\begin{abstract}
We find the GKO coset construction of the dimension 4 Casimir operator that contains the quartic WZW currents contracted with completely symmetric $S U(N)$ invariant tensors of ranks 4,3 , and 2 . The requirements, that the operator product expansion with the diagonal current is regular and it should be primary under the coset Virasoro generator of dimension 2, fix all the coefficients in spin- 4 current, up to two unknown coefficients. The operator product expansion of coset primary spin-3 field with itself fixes them completely. We compute the three-point functions with scalars for all values of the 't Hooft coupling in the large $N$ limit. At fixed 't Hooft coupling, these three-point functions are dual to that found by Chang and Yin recently in the undeformed $A d S_{3}$ bulk theory (higher spin gravity with matter).
\end{abstract}




\section{Introduction}

It was conjectured in [1] that the large $N$ limit of the $W_{N}$ (WZW coset) minimal model [2, 3, 4, 5] is dual to a particular $A d S_{3}$ higher spin theory of Vasiliev [6, 7, 8]. The bulk theory has an infinite tower of massless fields with spins $s=2,3, \cdots$ coupled to two complex scalars. The higher spin Lie algebra, where a free parameter $\lambda$ is present, describes interactions between the higher spin fields and the scalars. The scalars have equal mass determined by the algebra, $M^{2}=-\left(1-\lambda^{2}\right)$. They are quantized with opposite boundary conditions and their conformal dimensions are $h_{+}=\frac{1}{2}(1+\lambda)$ and $h_{-}=\frac{1}{2}(1-\lambda)$. The boundary theory is an $A_{N-1}$ (WZW coset) minimal model which has a higher spin $W_{N}$ symmetry generated by currents of spins 2,3, $\cdots, N$ [9]. See also [10] for a detailed description of $W_{N}$-symmetry in conformal field theory. The theory is labeled by two positive integers $N$ and $k$ where $k$ is the level of the WZW current algebra. The above bulk mass parameter is identified with the boundary 't Hooft coupling $\lambda=\frac{N}{N+k}$ which is fixed in the large $N$ limit.

Recently, in [11, the partition function of the $W_{N}$ minimal model was obtained. Due to the fact that certain states become null and decouple from correlation functions (and therefore have to be removed from the spectrum), the careful limiting procedure shows that the resulting states that survive exactly match the gravity prediction for all values of the 't Hooft coupling. In this computation, they have considered the 'strict' infinite $N$ limit where the sum of the number of boxes and antiboxes in the Young tableau has maximum value in the conformal field theory partition function. Furthermore, the zero mode eigenvalues (extension of the conformal dimensions) of the primary generators for spins $s=2,3,4$ are computed by using the classical Miura transformation (nonprimary basis) and transforming to primary basis, based on the work of [12]. Similarly, the eigenvalues in bulk higher spin algebra by using the Drinfeld-Sokolov reduction [13] are also computed with an appropriate limit. The degenerate representations of the two algebras match.

In [14, the three point functions with scalars at tree level in the undeformed bulk theory (with an appropriate normalization) are computed and then this result is compared to the three-point functions in the $W_{N}$ minimal model, in the large $N$ limit, at 't Hooft coupling $\lambda=\frac{1}{2}$. In particular, they have checked for spin-3 current and made predictions for the three-

point functions of spin $s \geq 4$, at fixed 't Hooft coupling $\lambda=\frac{1}{2}$, in the $W_{N}$ coset conformal field theory.

In this paper, we would like to compute the three-point functions for spin- 4 current in the $W_{N}$ minimal model, in the large $N$ limit, for all values of 't Hooft coupling. So, at first, one need to find spin-4 (or dimension-4) Casimir operator in the $W_{N}$ minimal model. So far, there 
are no known coset generators of spin greater than 3, although some partial attempts to this direction can be seen from the work of [15, 10]. The main obstacles to construct the coset generators of spin greater than 3 are that it is not straightforward to find the right candidate for the coset generator. For spin-3 generator, there are only four independent terms that can be written in terms of two kinds of WZW currents and then they are cubic in these currents. As a spin $s$ increases, the possible terms are increased very fast. Once a candidate for the higher spin generator is constructed, then one should discover all the coefficient functions, by using both

1) the operator product expansion of diagonal spin-1 current and higher spin generator should not contain any singular terms and

2 ) the higher spin generator should transform as a primary field of dimension-4 under the coset spin-2 Virasoro generator.

For the spin-3 case described in [5, 16], the only overall coefficient is not determined from the above two requirements. For the spin- 4 case in this paper, in general, there exist two unknown coefficients which depend on the levels and $N$. From the higher spin representations, one can find a linear equation between these two coefficients. Moreover, the operator product expansion of spin-3 with itself restricts to these two coefficients. It turns out that all the coefficients are fixed completely and we are left with the spin- 4 coset Casimir operator described in [5] (its explicit expression is not known so far and we present them for the first time in this paper).

Although there is a mathematica package for the operator product expansion by Thielemans [17] some time ago, one should perform all the computations by hand because one is interested in the case where the $N$ is arbitrary. Of course, for example, one can compute the operator product expansion for $N=4$ with $S U(4)$ group using his package and check whether the calculations by hand are correct or not. For all the computations on the operator product expansions in this paper, his package for $N=4$ has been used, as a consistency check 1 .

In section 2, after reviewing the basic contents on the two dimensional conformal field theory in the context of WZW model, we construct spin-4 Casimir operator using a completely symmetric traceless tensor $d$ symbol of rank 4. A generalization of [4, 5] to higher spin-4 current is described.

In section 3, after reviewing the Goddard, Kent and Olive (GKO) construction [2, 3], we find coset spin- 4 Casimir operator. There are twenty three independent terms. Along the line of [16], we describe the large $N$ limit of this coset spin-4 Casimir operator. We compute the

\footnotetext{
${ }^{1}$ Of course, the computations for $N=3$ are simpler than those for $N=4$, but the former does not tell us any nontrivial checks for the quartic Casimir operator because the $d$ symbol of rank 4 is zero identically. For the computations that do not involve this $d$ symbol, it is O.K. to take $N=3$ for consistency check.
} 
three-point functions with scalars and compare to the recent findings by Chang and Yin [14].

In section 4, we summarize what we have found in this paper and describe the relevant future works.

In the Appendices, we present all the details that are necessary to the sections 2, and 3.

There exist some relevant papers [18]-[38] which are related to the work of [1] and see also [39] where the quantum Miura transformation is introduced and the spins-3 and 4 primary fields are constructed from free massless scalar fields. Of course, these fields are quantum version of [12, 11], but it is not clear, at the moment, how the eigenvalues of a Casimir operator of order 4 have two arbitrary constants. It would be interesting to analyze the eigenvalues of the primary fields and to find the precise relations with the work of [1] further.

\section{The fourth-order Casimir operator of $S U(N)$}

\subsection{Review}

The conformal field $J(z)$ takes values in the Lie algebra $S U(N)$ and its components $J^{a}(z)(a=$ $\left.1,2, \cdots, N^{2}-1\right)$ with respect to an antihermitian basis $T^{a}$ where $\operatorname{Tr}\left(T^{a} T^{b}\right)=-\delta^{a b}$ satisfy the operator product expansion defined as [10]

$$
J^{a}(z) J^{b}(w)=-\frac{1}{(z-w)^{2}} k \delta^{a b}+\frac{1}{(z-w)} f^{a b c} J^{c}(w)+\cdots,
$$

where the positive integer $k$ is the level and $f^{a b c}$ are the structure constants of $S U(N)$. The dots stand for the regular terms in the limit $z \rightarrow w$.

The Sugawara stress energy tensor corresponding to the second order Casimir operator, in terms of currents $J^{a}(z)$, is given by

$$
T(z)=-\frac{1}{2(k+N)}\left(J^{a} J^{a}\right)(z),
$$

where the normal ordered field product denoted by brackets [10] is assumed. By using the operator product expansion (2.1), one can compute the operator product expansion between $T(z)$ and $T(w)$ and it turns out the well-known result 2

$$
T(z) T(w)=\frac{1}{(z-w)^{4}} \frac{c}{2}+\frac{1}{(z-w)^{2}} 2 T(w)+\frac{1}{(z-w)} \partial T(w)+\cdots .
$$

\footnotetext{
${ }^{2}$ Notice that the singular term $\frac{1}{(z-w)^{4}}$ in $(2.3)$ comes from the series expansions of $\frac{1}{(z-x)}$ and $\frac{1}{(z-x)^{2}}$ with respect to $x$ around $w$ where $x$ is an intermediate contour integral variable [4. This operator product expansion (2.3) can be written in terms of a commutator relation for the modes $L_{m}$ where $T(z)=\sum_{m \in \mathbf{Z}} \frac{L_{m}}{z^{m+2}}$ as follows: $\left[L_{m}, L_{n}\right]=\oint_{C_{0}} \frac{d w}{2 \pi i} w^{n+1} \oint_{C_{w}} \frac{d z}{2 \pi i} z^{m+1} T(z) T(w)=(m-n) L_{m+n}+\frac{c}{12} m\left(m^{2}-1\right) \delta_{m+n, 0}[10]$.
} 
The central charge $c$ is read off and it is found to be

$$
c=\frac{k}{k+N}\left(N^{2}-1\right)
$$

Furthermore, the current $J^{a}(z)$ is primary field of dimension 1 with respect to (2.2) as follows:

$$
T(z) J^{a}(w)=\frac{1}{(z-w)^{2}} J^{a}(w)+\frac{1}{(z-w)} \partial J^{a}(w)+\cdots .
$$

The standard way to obtain this operator product expansion (2.5) is to compute the operator product expansion between $J^{a}(z)$ and $T(w)$ first. Then one reverses the arguments $z$ and $w$ and uses the series expansion around $w$. Note $J^{a}(z) T(w)=\frac{1}{(z-w)^{2}} J^{a}(w)+\cdots$. The stress energy tensor (2.2) has dimension 2.

The third-order Casimir operator for $S U(N)$ is defined as [4]

$$
Q(z) \equiv d^{a b c}\left(J^{a}\left(J^{b} J^{c}\right)\right)(z)
$$

where $d^{a b c}$ are the $d$-symbols of $S U(N)$ and they are completely symmetric traceless $S U(N)$ invariant tensor of rank 33 . This is unique choice for the dimension 3 operator because the other choice $d^{a b c}\left(\left(J^{a} J^{b}\right) J^{c}\right)(z)$ reduces to the one (2.6) above by using the property (A.3) in this paper and the relation $(A .15)$ of [4].

Let us introduce the dimension 2 operator

$$
Q^{a}(z) \equiv d^{a b c} J^{b} J^{c}(z)
$$

For the computation of the operator product expansion $Q(z) Q(w)$, it is convenient to use this dimension 2 operator as well. The dimension 2 operator $f^{a b c} J^{b} J^{c}(z)$ is nothing but $N \partial J^{a}$ which can be obtained by using the identities (A.2) of this paper and (A.10) of [4]. Then it is straightforward to compute the following operator product expansions from the relations (2.6) and (2.7) [4]

$$
\begin{aligned}
J^{a}(z) Q^{b}(w) & =-\frac{1}{(z-w)^{2}}(2 k+N) d^{a b c} J^{c}(w)+\frac{1}{(z-w)} f^{a b c} Q^{c}(w)+\cdots \\
J^{a}(z) Q(w) & =-\frac{1}{(z-w)^{2}} 3(k+N) Q^{a}(w)+\cdots
\end{aligned}
$$

We have used the properties (A.6) and (A.10) in order to obtain the operator product expansion (2.8) and similarly, the relations (A.2) and (A.3) are used for the operator product expansion (2.9). One can compute the commutator, $\left[J_{0}^{a}, Q^{b}(w)\right]=\oint_{w} \frac{d z}{2 \pi i} J^{a}(z) Q^{b}(w)=f^{a b c} Q^{c}(w)$,

\footnotetext{
${ }^{3}$ From now on, we denote this normal ordered product without any brackets, for simplicity. That is, $\left(J^{a}\left(J^{b} J^{c}\right)\right)(z)=J^{a} J^{b} J^{c}(z)$. We follow the convention for the fully normal ordered product given in [4.
} 
where $J_{0}^{a}$ is a Fourier component $J_{0}^{a}=\oint \frac{d z}{2 \pi i} J^{a}(z)$ and the operator product expansion (2.8) is used. This implies that the field $Q^{a}(z)$ transforms under the adjoint representation of the horizontal finite dimensional Lie algebra $S U(N)$. Similarly, from the commutator, $\left[J_{0}^{a}, Q(w)\right]=0$, in which we used the fact that there is no $\frac{1}{(z-w)}$ term in the operator product expansion (2.9), the field $Q(z)$ transforms under the singlet of the horizontal finite Lie algebra $S U(N) 4$.

Furthermore, the fields $Q^{a}(z)$ and $Q(z)$ are primary fields of dimension 2 and 3, with respect to the stress energy tensor (2.2), respectively

$$
\begin{aligned}
T(z) Q^{a}(w) & =\frac{1}{(z-w)^{2}} 2 Q^{a}(w)+\frac{1}{(z-w)} \partial Q^{a}(w)+\cdots \\
T(z) Q(w) & =\frac{1}{(z-w)^{2}} 3 Q(w)+\frac{1}{(z-w)} \partial Q(w)+\cdots
\end{aligned}
$$

where we have used the properties (A.1) and (A.3) in order to simplify the terms $\frac{1}{(z-w)^{4}}$ and $\frac{1}{(z-w)^{3}}$ in (2.10) respectively. For the computation of the operator product expansion (2.11), we have used the fact that there is no singular term in the operator product expansion $J^{a}(z) Q^{a}(w)$ from the relation (2.8) and this is the reason why there are no higher order singular terms in the equation (2.11).

The singlet algebra (or Casimir algebra) of primary fields of dimension $3, Q(z)$, and dimension 2, $T(z)$, together with the operator product expansions (2.3) and (2.11), can be obtained by computing the operator product expansion $Q(z) Q(w)$ explicitly [4]. In general, the new primary field of dimension 4 arises in this operator product expansion. However, for $N=3, k=1$, the new spin 4 primary field vanishes identically.

\subsection{Fourth order Casimir}

How one can determine the fourth-order Casimir of $S U(N)$ with $N>3$ ? The completely symmetric traceless $d$ symbols are introduced by Schoutens in [40] (See also [41]) as follows:

$$
d^{a b c d}=d^{a b e} d^{e c d}+d^{a c e} d^{e b d}+d^{a d e} d^{e b c}-\frac{4\left(N^{2}-4\right)}{N\left(N^{2}+1\right)}\left(\delta^{a b} \delta^{c d}+\delta^{a c} \delta^{b d}+\delta^{a d} \delta^{b c}\right) .
$$

The $N$-dependent coefficient above can be fixed by requiring that this $d$ tensor of rank 4 should be traceless. When the indices $a, b$ are equal, $b=a$, the first term of (2.12) vanishes due to the property (A.1), the second and third terms of (2.12) contribute to $\frac{4}{N}\left(N^{2}-4\right)$ via the identity (A.4), the fourth term contains $\left(N^{2}-1\right)$ and the fifth and sixth terms of (2.12)

\footnotetext{
${ }^{4}$ Also the stress energy tensor $T(z)$ is a singlet under the horizontal (finite-dimensional) subalgebra $S U(N)$ of $\widehat{S U}(N)$ because the operator product expansion between $J^{a}(z)$ and $T(w)$ does not contain $\frac{1}{(z-w)}$ term as before and the commutator $\left[J_{0}^{a}, T(w)\right]$ vanishes.
} 
contribute to 1 . Therefore, the requirement $d^{\text {aacd }}=0$ implies that the $N$ dependent coefficient should be $-\frac{4\left(N^{2}-4\right)}{N\left(N^{2}+1\right)}$. The symmetric property between the indices of $d^{a b c d}$ can be seen from the relation (2.12) by using the symmetry in the symmetric tensors $d^{a b c}$ and $\delta^{a b}$. The higher order $d$ tensors of rank greater than 4 are also introduced in [42] 5 .

There were some attempts, by Watts in [15] and by Bouwknegt and Schoutens in the preprint version of [10], to the generalization for the coset spin-3 generator. It originates from the equation (2.12) of [43], but this identity is not valid. For the spin-3 generator, the $N$-dependence of $\frac{1}{(z-w)^{2}}$ term in the operator product expansion (2.8) was obtained by using the relation (A.6). The contraction with one $d$ symbol and two $f$ symbols leads to other $d$ symbol, but this is not true for higher $d$ symbol. For example, the triple product $d^{a b c d} f^{f g a} f^{f h b}$ does not produce the $d^{\text {ghcd }}$ tensor of rank 4 exactly, but there exist some extra structures. This can be checked explicitly by taking the 4-th order $d$ symbol in (2.12) with two $f$ symbols. So far, there are no known coset generators of spin greater than 3.

It is convenient to introduce the following dimension 1,2,3 and 4 operators, as in spin-3 generator, along the line of [10]:

$$
\begin{aligned}
T_{a b c}^{(1,3)}(z) & \equiv d^{a b c d} J^{d}(z), \\
T_{a b}^{(2,2)}(z) & \equiv d^{a b c d} J^{c} J^{d}(z), \\
T_{a}^{(3,1)}(z) & \equiv d^{a b c d} J^{b} J^{c} J^{d}(z), \\
T^{(4,0)}(z) & \equiv d^{a b c d} J^{a} J^{b} J^{c} J^{d}(z) .
\end{aligned}
$$

Here $d$ symbol is given by (2.12). It turns out that they are primary fields of dimension $1,2,3$ and 4 with respect to the stress energy tensor $(2.2)$ :

$$
\begin{aligned}
T(z) T_{a b c}^{(1,3)}(w) & =\frac{1}{(z-w)^{2}} T_{a b c}^{(1,3)}(w)+\frac{1}{(z-w)} \partial T_{a b c}^{(1,3)}(w)+\cdots \\
T(z) T_{a b}^{(2,2)}(w) & =\frac{1}{(z-w)^{2}} 2 T_{a b}^{(2,2)}(w)+\frac{1}{(z-w)} \partial T_{a b}^{(2,2)}(w)+\cdots \\
T(z) T_{a}^{(3,1)}(w) & =\frac{1}{(z-w)^{2}} 3 T_{a}^{(3,1)}(w)+\frac{1}{(z-w)} \partial T_{a}^{(3,1)}(w)+\cdots \\
T(z) T^{(4,0)}(w) & =\frac{1}{(z-w)^{2}} 4 T^{(4,0)}(w)+\frac{1}{(z-w)} \partial T^{(4,0)}(w)+\cdots
\end{aligned}
$$

\footnotetext{
${ }^{5}$ Let us comment on the notations for the $d$ symbol and $f$ symbol we are using in the paper. We follow the convention of [4. There are some differences between the notations in [4, 5] and [10. For example, the minus sign in the third equation of (7.23) of $[10$ and some minus signs in the equation (7.22) of [10]. The normalization of $\sqrt{2}$ between [42] and [4, 5] appears. We insert the Jacobi identity which was missed in [4, 5] into the identity (A.11) of the Appendix $A$. We have checked that for $N=3$, the quantity on the right hand side of (2.12) is zero identically, by using the explicit $f$ tensor and $d$ tensor elements with a factor of $\sqrt{2}$ in 42]. For the nontrivial $d$ tensor of rank 4 , the $N$ should be greater than 3 .
} 
It is easy to see the operator product expansion (2.17) by using the defining equation (2.5). The higher order singular terms in the operator product expansion (2.18) vanish due to the fact that the products of $d^{a b c d}$ and $\delta^{c d}$ or $f^{c d e}$ contribute to zero. Also the higher order singular terms in the operator product expansion (2.19) do not occur because the operator product expansion of $J^{b}(z) T_{a b}^{(2,2)}(w)$ does not contain any singular terms (note that the $d^{a b c d}$ symbol is symmetric and traceless). Similarly, there are no higher order singular terms in the operator product expansion (2.20) because the operator product expansion between $J^{a}(z)$ and $T_{a}^{(3,1)}(w)$ does not produce any singular terms.

Now it is ready to compute the operator product expansions of $J^{a}(z)$ and four primary fields (2.13), (2.14), (2.15) and (2.16), as we did in the subsection 2.1. Let us present them here as follows:

$$
\begin{aligned}
J^{a}(z) T_{b c d}^{(1,3)}(w) & =-\frac{1}{(z-w)^{2}} k d^{a b c d}+\frac{1}{(z-w)} f^{a e f} d^{b c d e} J^{f}(w)+\cdots, \\
J^{a}(z) T_{b c}^{(2,2)}(w) & =\frac{1}{(z-w)^{2}}\left[-2 k d^{a b c d} J^{d}(w)+f^{a d f} f^{f e g} d^{b c d e} J^{g}(w)\right] \\
& +\frac{1}{(z-w)}\left[f^{a d f} d^{b c d e} J^{f} J^{e}(w)+f^{a e f} d^{b c d e} J^{d} J^{f}(w)\right]+\cdots, \\
J^{a}(z) T_{b}^{(3,1)}(w) & =\frac{1}{(z-w)^{3}} f^{a c f} d^{b c d e} f^{g e h} f^{f d g} J^{h}(w)+\frac{1}{(z-w)^{2}}\left[-3 k d^{a b c d} J^{c} J^{d}(w)\right. \\
& \left.+f^{a c f} f^{f d g} d^{b c d e} J^{g} J^{e}(w)+f^{a c f} f^{f e g} d^{b c d e} J^{d} J^{g}(w)+f^{a d f} f^{f e g} d^{b c d e} J^{c} J^{g}(w)\right] \\
& +\frac{1}{(z-w)}\left[f^{a c f} d^{b c d e} J^{f} J^{d} J^{e}(w)+f^{a d f} d^{b c d e} J^{c} J^{f} J^{e}(w)+f^{a e f} d^{b c d e} J^{c} J^{d} J^{f}(w)\right] \\
& +\cdots, \\
& =\frac{1}{(z-w)^{4}} f^{a b f} f^{f c i} d^{b c d e} f^{g e h} f^{i d g} J^{h}(w) \\
& +\frac{1}{(z-w)^{3}}\left[f^{a b f} d^{b c d e}\left(f^{h d g} f^{f c h} J^{g} J^{e}(w)+f^{h e g} f^{f c h} J^{d} J^{g}(w)+f^{h e g} f^{f d h} J^{c} J^{g}(w)\right)\right. \\
& \left.+f^{a c f} d^{b c d e} f^{g e h} f^{f d g} J^{b} J^{h}(w)\right] \\
& +\frac{1}{(z-w)^{2}}\left[-4 k d^{a b c d} J^{b} J^{c} J^{d}(w)+f^{a b f} f^{f c g} d^{b c d e} J^{g} J^{d} J^{e}(w)\right. \\
& +f^{a b f} f^{f d g} d^{b c d e} J^{c} J^{g} J^{e}(w)+f^{a b f} f^{f e g} d^{b c d e} J^{c} J^{d} J^{g}(w)+f^{a c f} f^{f d g} d^{b c d e} J^{b} J^{g} J^{e}(w) \\
& \left.+f^{a c f} f^{f e g} d^{b c d e} J^{b} J^{d} J^{g}(w)+f^{a d f} f^{f e g} d^{b c d e} J^{b} J^{c} J^{g}(w)\right]+\cdots
\end{aligned}
$$

It is easy to see the operator product expansion (2.21) from the defining equation (2.1). There is no $\frac{1}{(z-w)^{3}}$ term in the operator product expansion (2.22) because the product of $d^{b c d e}$ and $f^{a d e}$ vanishes.

In the operator product expansion (2.23), the result of the operator product expansion 
(2.22) is used and the $k$-dependent term in the singular term of $\frac{1}{(z-w)^{3}}$ vanishes due to the fact that the product of $d^{f b c d}$ and $f^{a c f}$ becomes zero. One need to simplify the singular term of $\frac{1}{(z-w)^{3}}$ further in the operator product expansion (2.23). In order to simplify this, one should use the $d$ symbol in (2.12). By plugging the first term of (2.12) into the above singular term, $f d f f$ term, one realizes that $d f f$ term can be reexpressed in terms of a single $d$ term via the identity (A.6). Then this reduces to the product of $d d f$ and this leads to a single $f$ term from the relation (A.7). Finally, one obtains $-N d^{\text {bha }}$ coming from the first term of (2.12) 6. Now let us consider the second term in (2.12). In the above singular term, this provides $d d f f$ term which can be further reduced to the relation (A.14). With the remaining $f$ in $\frac{1}{(z-w)^{3}}$ term, it leads to a single $f$ term and $d d f$ terms. These $d d f$ terms can be simplified further by using the relation (A.7). It turns out that the final expression coming from the second term of (2.12) is equal to zero. For the third term of (2.12), the singular term can be written similarly. It gives $-\left(N^{2}-4\right) f^{a b h}$. Finally, the $\delta$ terms in (2.12) can be reduced to $f f f$ terms and these can be written in terms of a single $f$ terms by the identity (A.5).

By collecting all the contributions we have obtained so far, the $\frac{1}{(z-w)^{3}}$ term in the operator product expansion (2.23) can be summarized by $-\frac{2\left(N^{2}-4\right)\left(N^{2}-9\right)}{\left(N^{2}+1\right)} f^{a b c} J^{c}(w)$. The other lower singular terms are simplified further and the details for these computations are presented in the Appendix B. The complete operator product expansion (2.23) is presented in (B.10).

Moreover, in the operator product expansion (2.24), the result of the operator product expansion (2.23) is also used in the $\frac{1}{(z-w)^{4}}$ and $\frac{1}{(z-w)^{3}}$ terms. Let us consider the highest singular term, $\frac{1}{(z-w)^{4}}$ term. By substituting the $d$ symbol (2.12) into there, one has $f f d d f f$ terms. One uses the identity (A.6) and obtains $2 N\left(N^{2}-4\right) \delta^{a h}$ coming from the first term of (2.12). The second term of (2.12) with other four $f$ symbols can be simplified via the identity (A.14) with the relation (A.7). It turns out that there is no contribution. The third term of (2.12) gives a nonzero contribution. We again use the identity (A.14) with (A.7) and (A.2) and the final expression is $2 N\left(N^{2}-4\right) \delta^{a h}$. The remaining terms of (2.12) contribute to $-\frac{40 N\left(N^{2}-4\right)}{\left(N^{2}+1\right)}$ where the identities (A.2) and (A.5) are used. Then, one obtains $\frac{4 N\left(N^{2}-4\right)\left(N^{2}-9\right)}{\left(N^{2}+1\right)} J^{a}(w)$ in $\frac{1}{(z-w)^{4}}$ term.

Let us consider the next higher order singular term, $\frac{1}{(z-w)^{3}}$. The first term of $d$ symbol in (2.12) contributes to $\left(N^{2}-4\right) f^{e a g}$ via the identities (A.6) and (A.7). There is no contribution from the second term of $d$ symbol where the identities (A.14) and (A.7) are used. The third term of (2.12) gives $\left(N^{2}-4\right) f^{a g e}$ via the relations (A.14) and (A.7). The delta terms of $d$ symbol can be simplified, through the identities (A.5) and (A.2), as $5 N f^{a g e}$ with prefactor $-\frac{4\left(N^{2}-4\right)}{N\left(N^{2}+1\right)}$. By summing over all the contributions, one obtains $\frac{2 N\left(N^{2}-4\right)\left(N^{2}-9\right)}{\left(N^{2}+1\right)} \partial J^{a}(w)$ in $\frac{1}{(z-w)^{3}}$.

\footnotetext{
${ }^{6}$ Here we have ignored the index structures of $d$ and $f$ symbols for simplicity.
} 
The next term can be arranged as the sum of $f d f f$ term above and $f d f f f$ term by moving the current $J^{g}$ to the left. This last term can be further simplified as $-\frac{4 N\left(N^{2}-4\right)\left(N^{2}-9\right)}{\left(N^{2}+1\right)} \partial J^{a}(w)$. The $J^{c} J^{g}$ term is the same as the $J^{d} J^{g}$ term above by interchanging between the indices $c$ and $d$ that are dummy indices. The $J^{b} J^{h}$ term in $\frac{1}{(z-w)^{3}}$ can be written as the sum of above first contribution and $-\frac{4 N\left(N^{2}-4\right)\left(N^{2}-9\right)}{\left(N^{2}+1\right)} \partial J^{a}(w)$ term.

Therefore, one has the final term as $-\frac{4 N\left(N^{2}-4\right)\left(N^{2}-9\right)}{\left(N^{2}+1\right)} \partial J^{a}(w)$ in the $\frac{1}{(z-w)^{3}}$. The other terms in lower singular terms are simplified further and the details for these computations are also presented in the Appendix B. The full operator product expansion (2.24) is presented in $(\underline{B} .13)$. There are no $\frac{1}{(z-w)}$ terms in the operator product expansion (2.24) for $N=4$. We expect this is also true for any $N>5$. The singlet field $T^{(4,0)}(z)$ has a vanishing commutator $\left[J_{0}^{a}, T^{(4,0)}(w)\right]=0$ under $S U(N)$ because there is no $\frac{1}{(z-w)}$ term in the operator product expansion (2.24).

The higher order singular terms, $\frac{1}{(z-w)^{4}}$ term and $\frac{1}{(z-w)^{3}}$ term, in the operator product expansions (2.23) and (2.24) allow us to consider the extra terms for the coset spin 4 current in the coset construction which will be discussed in next section. We also present the operator product expansions between $J^{a}(z)$ and other spin 4 fields in the Appendix $C$.

We have seen that the field $T^{(4,0)}$ is primary field of dimension 4 from the operator product expansion (2.20). However, for general spin 4 field, the condition for primary field under the stress energy tensor (2.2) is not straightforward. Sometimes the higher order singular terms arise in the operator product expansion with the stress energy tensor $T(z)$. For example, one can think of spin 4 field $Q^{a} Q^{a}(z)$ which is quadratic in dimension 2 field $Q^{a}(z)$ (2.7). In order to compute the operator product expansion $T(z) Q^{a} Q^{a}(w)$ explicitly, one should obtain the operator product expansion $Q^{a}(z) Q^{a}(w)$ and more generally, one has the following operator product expansion with different indices:

$$
\begin{aligned}
Q^{a}(z) Q^{b}(w) & =\frac{1}{(z-w)^{4}} \frac{2}{N}\left(N^{2}-4\right) k(N+2 k) \delta^{a b}-\frac{1}{(z-w)^{3}} \frac{2}{N}\left(N^{2}-4\right)(N+2 k) f^{a b c} J^{c}(w) \\
& -\frac{1}{(z-w)^{2}}\left[N d^{a b c} Q^{c}(w)+2(N+2 k) d^{a c e} d^{b d e} J^{c} J^{d}(w)\right] \\
& +\frac{1}{(z-w)}\left[-(N+2 k) d^{a c e} d^{b d e} \partial J^{c} J^{d}(w)+f^{a c e} d^{b c d} Q^{e} J^{d}(w)+f^{a d e} d^{b c d} J^{c} Q^{e}(w)\right. \\
& \left.-(N+2 k) d^{a c e} d^{b d e} J^{d} \partial J^{c}(w)\right]+\cdots
\end{aligned}
$$

Here one uses the identity $(A .8)$ of [4] with the operator product expansion (2.8) and obtains the operator product expansion $Q^{a}(z) J^{c}(w)$. In order to have the complete expression for the higher order singular terms, one uses the relations (A.4 and (A.7).

It is ready to write down the operator product expansion $T(z)$ with $Q^{a} Q^{a}(w)$ and it turns 
out to be

$$
\begin{aligned}
T(z) Q^{a} Q^{a}(w) & =\frac{1}{(z-w)^{6}} \frac{12 k}{N}\left(N^{2}-4\right)\left(N^{2}-1\right)(N+2 k) \\
& +\frac{1}{(z-w)^{4}} \frac{32}{N}\left(N^{2}-4\right)(N+2 k)(N+k) T(w) \\
& +\frac{1}{(z-w)^{3}} \frac{12}{N}\left(N^{2}-4\right)(N+2 k)(N+k) \partial T(w) \\
& +\frac{1}{(z-w)^{2}} 4 Q^{a} Q^{a}(w)+\frac{1}{(z-w)} \partial\left(Q^{a} Q^{a}\right)(w)+\cdots
\end{aligned}
$$

The higher order singular terms in the operator product expansion (2.26) are determined by the operator product expansion (2.25) and the identity (A.4). These higher order terms will play an important role in next section. We should find the correct candidate for the coset spin 4 field which transforms as a primary field of dimension 4 under the coset Virasoro field. In other words, the higher order singular terms $\frac{1}{(z-w)^{n}}$ with $n>2$ in the operator product expansion between the coset Virasoro field and the coset spin 4 field should vanish. We also present the operator product expansions between $T(z)$ and the other spin 4 fields in the Appendix $C$.

The singlet algebra (or Casimir algebra) of primary fields $T^{(4,0)}(z), Q(z)$, and $T(z)$, can be obtained, in principle, by computing the operator product expansions $Q(z) T^{(4,0)}(w)$ and $T^{(4,0)}(z) T^{(4,0)}(w)$ explicitly. In general, we expect that the new primary fields will arise in these operator product expansions. For particular values of $N, k$, these new primary fields will vanish and the algebra reduces to [44, 45], along the line of [4]. Of course, it would be interesting to perform all the operator product expansions to see how the algebra is different from the algebra in [44, 45], but we are interested in a minimal model conformal field theory. In next section, we want to construct a new primary field of dimension 4, based on the operator product expansions we have described in this section.

Therefore, we have seen the fourth-order Casimir operator $d^{a b c d} J^{a} J^{b} J^{c} J^{d}(z)$ which will play an important role in next section.

\section{The GKO coset construction}

\subsection{Review}

Let us consider the diagonal coset WZW model given by

$$
\frac{\widehat{S U}(N)_{k} \oplus \widehat{S U}(N)_{1}}{\widehat{S U}(N)_{k+1}}
$$


Denoting the spin 1 current fields as $K^{a}(z)$ and $J^{a}(z)$ of level $k$ and 1 respectively and the spin 1 current field as $J^{\prime a}(z)$ of level $k+1$, the operator product expansion between $J^{a}(z)$ and $J^{b}(w)$ is

$$
J^{a}(z) J^{b}(w)=-\frac{1}{(z-w)^{2}} k_{1} \delta^{a b}+\frac{1}{(z-w)} f^{a b c} J^{c}(w)+\cdots
$$

where the level is characterized by $k_{1}(\equiv 1)$ and the other operator product expansion between $K^{a}(z)$ and $K^{b}(w)$ is

$$
K^{a}(z) K^{b}(w)=-\frac{1}{(z-w)^{2}} k_{2} \delta^{a b}+\frac{1}{(z-w)} f^{a b c} K^{c}(w)+\cdots,
$$

where the level is given by $k_{2}(\equiv k)$. We follow the convention of [16]. These two currents are independent in the sense that the operator product expansion $J^{a}(z) K^{b}(w)=0$. That is, there are no singular terms. The diagonal current is given by

$$
J^{\prime a}(z)=J^{a}(z)+K^{a}(z)
$$

Then it is easy to check, by adding the operator product expansions (3.2) and (3.3), that

$$
J^{\prime a}(z) J^{\prime b}(w)=-\frac{1}{(z-w)^{2}} k^{\prime} \delta^{a b}+\frac{1}{(z-w)} f^{a b c} J^{\prime c}(w)+\cdots, \quad k^{\prime} \equiv k_{1}+k_{2} \equiv k+1 .
$$

The stress energy tensor for the coset model (3.1) is given by

$$
\widetilde{T}(z)=T_{(1)}(z)+T_{(2)}(z)-T^{\prime}(z)
$$

where the stress energy tensors in terms of the spin 1 currents are as follows:

$$
\begin{aligned}
T_{(1)}(z) & =-\frac{1}{2\left(k_{1}+N\right)}\left(J^{a} J^{a}\right)(z) \\
T_{(2)}(z) & =-\frac{1}{2\left(k_{2}+N\right)}\left(K^{a} K^{a}\right)(z) \\
T^{\prime}(z) & =-\frac{1}{2\left(k_{1}+k_{2}+N\right)}\left(J^{\prime a} J^{\prime a}\right)(z) .
\end{aligned}
$$

It is easy to check that the operator product expansion between $J^{\prime a}(z)$ and $\widetilde{T}(w)$ has no singular term because the $\frac{1}{(z-w)^{2}}$ term is given by $\left(J^{a}(w)+K^{a}(w)-J^{\prime a}(w)\right)$ which is identically zero from (3.4). One computes the operator product expansion $\widetilde{T}(z) \widetilde{T}(w)$ that is equivalent to the operator product expansion $\widetilde{T}(z)\left(T_{(1)}+T_{(2)}\right)(w)$ because there is no singular term in the operator product expansion between $\widetilde{T}(z)$ and $T^{\prime}(w)$, by construction. Then by substituting (3.6) into the operator product expansion $\widetilde{T}(z)\left(T_{(1)}+T_{(2)}\right)(w)$, one has 
$T_{(1)}(z) T_{(1)}(w)+T_{(2)}(z) T_{(2)}(w)-T^{\prime}(z) T^{\prime}(w)$. Here we used the fact that the operator product expansion of $T^{\prime}(z)\left(T_{(1)}+T_{(2)}\right)(w)$ can be rewritten as $T^{\prime}(z)\left(\widetilde{T}(w)+T^{\prime}(w)\right)$ and this becomes $T^{\prime}(z) T^{\prime}(w)$.

Therefore, one obtains 7

$$
\begin{aligned}
\widetilde{T}(z) \widetilde{T}(w) & =T_{(1)}(z) T_{(1)}(w)+T_{(2)}(z) T_{(2)}(w)-T^{\prime}(z) T^{\prime}(w), \\
& =\frac{1}{(z-w)^{4}} \frac{\widetilde{c}}{2}+\frac{1}{(z-w)^{2}} 2 \widetilde{T}(w)+\frac{1}{(z-w)} \partial \widetilde{T}(w)+\cdots .
\end{aligned}
$$

Finally, after reading off the $\frac{1}{(z-w)^{4}}$ terms in (3.8), one has the following coset central charge as follows:

$$
\begin{aligned}
\widetilde{c} & =c_{(1)}+c_{(2)}-c^{\prime}=\left(N^{2}-1\right)\left(\frac{k_{1}}{k_{1}+N}+\frac{k_{2}}{k_{2}+N}-\frac{k_{1}+k_{2}}{k_{1}+k_{2}+N}\right) \\
& \equiv(N-1)\left[1-\frac{N(N+1)}{(N+k)(N+k+1)}\right],
\end{aligned}
$$

where we put $k_{1}=1, k_{2}=k$.

It is known that the coset spin 3 primary field $\widetilde{T}^{(3)}(z)$ is found in [5]. Together with (3.8), the operator product expansion of $\widetilde{T}(z) \widetilde{T}^{(3)}(z)$ satisfies the standard one for the primary field of dimension 3 and the operator product expansion of $\widetilde{T}^{(3)}(z) \widetilde{T}^{(3)}(w)$ provides the full structure of the operator algebra. The spin 3 field $\widetilde{T}^{(3)}(z)$ consists of four terms which can be written as the spin 1 currents $J^{a}(z), K^{a}(z)$. The requirement that the field $\widetilde{T}^{(3)}(z)$ is a primary field of dimension 3 under the $\widetilde{T}(z)$ fixes $\widetilde{T}^{(3)}(z)$ up to a normalization factor which depends on $N, k_{1}$ and $k_{2}$.

\subsection{Coset primary spin- 4 current $\widetilde{W}(z)$}

Along the line of coset spin 3 current [5], one can think of the following fields as a candidate for the coset spin 4 current

$$
d^{a b c d}\left(c_{1} J^{a} J^{b} J^{c} J^{d}+c_{2} J^{a} J^{b} J^{c} K^{d}+c_{3} J^{a} J^{b} K^{c} K^{d}+c_{4} J^{a} K^{b} K^{c} K^{d}+c_{5} K^{a} K^{b} K^{c} K^{d}\right),
$$

where the coefficient functions $c_{i}(i=1,2, \cdots 5)$ depend on $N, k_{1}$ and $k_{2}$. We simply generalize the field $T^{(4,0)}(z)$ to have both spin 1 currents, $J^{a}(z)$ and $K^{a}(z)$. How does one determine the coset spin 4 primary field? As we described in the introduction, first of all, the coset spin 4 primary field should commmute with the diagonal current (3.4) as follows:

$$
J^{\prime a}(z) \widetilde{W}(w)=\text { regular. }
$$

\footnotetext{
7 The stress energy tensor $T_{(1)}(z)$ has a central charge $c_{(1)}=\left(N^{2}-1\right) \frac{k_{1}}{N+k_{1}}(\underline{2.4})$, the stress energy tensor $T_{(2)}(z)$ has a central charge $c_{(2)}=\left(N^{2}-1\right) \frac{k_{2}}{N+k_{2}}$, and the stress energy tensor $T^{\prime}(z)$ has a central charge $c^{\prime}=\left(N^{2}-1\right) \frac{k_{1}+k_{2}}{N+k_{1}+k_{2}}$ with (3.5).
} 
Secondly, the coset spin 4 primary field should transform as dimension 4 under the stress energy tensor (3.6) as follows:

$$
\widetilde{T}(z) \widetilde{W}(w)=\frac{1}{(z-w)^{2}} 4 \widetilde{W}(w)+\frac{1}{(z-w)} \partial \widetilde{W}(w)+\cdots .
$$

One can easily see that if there exist only five candidate terms as in (3.10), then there are no consistent coefficient functions satisfying the conditions (3.11) by checking the operator product expansions given in the Appendix (D.1)-(D.5). This is one indication why the construction in [15] where the field contents are given by (3.10) is not correct. Therefore, one should consider the extra terms in order to satisfy (3.11) by requiring that they contribute to the vanishing of any singular terms. Since the $d$ symbol of rank 4 is defined as (2.12), one also considers the following candidates for the coset spin 4 primary field by looking at the first term of (2.12)

$$
d^{a b e} d^{c d e}\left(c_{6} J^{a} J^{b} J^{c} J^{d}+c_{7} J^{a} J^{b} J^{c} K^{d}+c_{8} J^{a} J^{b} K^{c} K^{d}+c_{9} J^{a} K^{b} K^{c} K^{d}+c_{10} K^{a} K^{b} K^{c} K^{d}\right) .(3
$$

For given the first term in (3.13), we simply consider the possible terms with the current $K^{a}(z)$ as we did in (3.10). Then it is ready to check whether the sum of (3.10) and (3.13) is consistent with the condition (3.11) or not. It turns out that they do not satisfy the regularity condition (3.11). This implies that we need to have more terms and we will have a chance to remove all the singular terms. The delta term in (2.12) gives us to allow the following terms

$$
c_{11} J^{a} J^{a} J^{b} J^{b}+c_{12} J^{a} J^{a} J^{b} K^{b}+c_{13} J^{a} J^{a} K^{b} K^{b}+c_{14} J^{a} K^{a} K^{b} K^{b}+c_{15} K^{a} K^{a} K^{b} K^{b} .
$$

For given first term in (3.14), we also consider the possible terms by realizing the other current $K^{a}(z)$. Still the contractions in the indices are fixed. Moreover, one should also consider the following terms

$$
\begin{aligned}
& c_{16} \partial^{2} J^{a} J^{a}+c_{17} \partial^{2} J^{a} K^{a}+c_{18} \partial^{2} K^{a} K^{a}+c_{19} \partial J^{a} \partial J^{a}+c_{20} \partial J^{a} \partial K^{a}+c_{21} \partial K^{a} \partial K^{a} \\
& +c_{22} J^{a} \partial^{2} K^{a} .
\end{aligned}
$$

These terms appear in the second derivative of coset stress energy tensor (3.7).

By adding the terms (3.10), (3.13), (3.14), and (3.15), one writes down the following coset spin 4 current

$$
\begin{aligned}
\widetilde{W}(z) & =d^{a b c d}\left(c_{1} J^{a} J^{b} J^{c} J^{d}+c_{2} J^{a} J^{b} J^{c} K^{d}+c_{3} J^{a} J^{b} K^{c} K^{d}+c_{4} J^{a} K^{b} K^{c} K^{d}+c_{5} K^{a} K^{b} K^{c} K^{d}\right) \\
& +d^{a b e} d^{c d e}\left(c_{6} J^{a} J^{b} J^{c} J^{d}+c_{7} J^{a} J^{b} J^{c} K^{d}+c_{8} J^{a} J^{b} K^{c} K^{d}+c_{9} J^{a} K^{b} K^{c} K^{d}+c_{10} K^{a} K^{b} K^{c} K^{d}\right) \\
& +c_{11} J^{a} J^{a} J^{b} J^{b}+c_{12} J^{a} J^{a} J^{b} K^{b}+c_{13} J^{a} J^{a} K^{b} K^{b}+c_{14} J^{a} K^{a} K^{b} K^{b}+c_{15} K^{a} K^{a} K^{b} K^{b} \\
& +c_{16} \partial^{2} J^{a} J^{a}+c_{17} \partial^{2} J^{a} K^{a}+c_{18} \partial^{2} K^{a} K^{a}+c_{19} \partial J^{a} \partial J^{a}+c_{20} \partial J^{a} \partial K^{a} \\
& +c_{21} \partial K^{a} \partial K^{a}+c_{22} J^{a} \partial^{2} K^{a} .
\end{aligned}
$$


We also checked whether the other contractions with different indices provide other independent terms we are missing or not in the Appendix G. In particular, the equations (G.1), (G.2), (G.3) and (G.4). It turns out that there are no other independent terms except the field $J^{a} J^{b} K^{a} K^{b}(z)$. We will discuss on the presence of this field later. The field contents in (3.16) are complete except the field $J^{a} J^{b} K^{a} K^{b}(z)$ in this sense. Due to the fact that there exists an identity

$$
\begin{aligned}
& d^{a b c d} J^{a} J^{b} J^{c} J^{d}(z)=3 d^{a b e} d^{c d e} J^{a} J^{b} J^{c} J^{d}(z)-\frac{12\left(N^{2}-4\right)}{N\left(N^{2}+1\right)} J^{a} J^{a} J^{b} J^{b}(z) \\
& -\frac{3\left(N^{2}-4\right)\left(N^{2}-3\right)}{N^{2}+1} \partial J^{a} \partial J^{a}(z)+\frac{2\left(N^{2}-4\right)\left(N^{2}-3\right)}{N^{2}+1} \partial^{2} J^{a} J^{a}(z),
\end{aligned}
$$

and the terms appearing in the coefficients $c_{1}, c_{6}, c_{11}, c_{16}$ and $c_{19}$ (and similarly the coefficients $c_{5}, c_{10}, c_{15}, c_{18}$ and $\left.c_{21}\right)$ are not independent. For example, this allows us to put $c_{19}=0=c_{21}$ from the beginning in order to keep the quartic term on the left hand side of (3.17).

Let us apply the condition (3.11) to the coset spin 4 current (3.16). In Appendix D, we present the twenty two operator product expansions (D.1) $-(\overline{D .22})$. Next we consider the property of (3.12) on the coset spin 4 current. Since we require the condition (3.11) and the stress energy tensor $T^{\prime}(z)$ is given by (3.7), one has no singular terms in the operator product expansion $T^{\prime}(z)$ and $\widetilde{W}(w)$. This implies that we introduce

$$
T_{(1)}(z)+T_{(2)}(z) \equiv \hat{T}(z),
$$

and consider the operator product expansion with $\widetilde{W}(w)$. We collect the operator product expansions in the Appendix E: (E.2)-(E.23). Then the twenty three linear equations from the vanishing of singular terms in the operator product expansions $J^{\prime a}(z) \widetilde{W}(w)$ are summarized in the equations ( $(\underline{F .1}),(\underline{F .3})$ and (F.4) of Appendix F. Similarly, the eight linear equations from the vanishing of higher order singular terms (i.e., $\frac{1}{(z-w)^{n}}$ term with $n>2$ ) in the operator product expansions $\hat{T}(z) \widetilde{W}(w)$ are given in the equations (F.5), (F.6) and (F.7) of the Appendix $F$. By solving these equations, the coefficients appearing in the coset spin 4 current (3.16) are determined except the $c_{1}$ coefficient and they are in the equations (F.8) and (F.9) of the Appendix $F$. The large $N$ limit for these coefficients is given in (F.10) that will be used in next subsection.

\subsection{Coset primary spin-4 current $\widetilde{W}(z)$ in the large $N$ 't Hooft limit}

The large $N$ 't Hooft limit [1] is defined as

$$
N, k \rightarrow \infty, \quad \lambda \equiv \frac{N}{N+k} \quad \text { fixed. }
$$


Let us describe the large $N$ 't Hooft limit for the coset spin 4 current (3.16) with the coefficients (F.8) and (F.9). In this limit, the term proportional to $d^{a b c d} K^{a} K^{b} K^{c} K^{d}(z), c_{5}$, behaves as $\frac{1}{N}$ and goes to zero. Similarly, the term with $d^{a b e} d^{c d e} K^{a} K^{b} K^{c} K^{d}(z), c_{10}$, behaves as $\frac{1}{N}$ also and vanishes. The terms with $c_{11}, c_{12}$ vanish, the terms with $c_{13}, c_{14}$ have $\frac{1}{N}$ dependence and the term with $c_{15}$ has $\frac{1}{N^{2}}$ dependence. See the relations (F.10) for details. Then, all of these vanish in the large $N$ limit. One obtains the coset spin 4 current, in the large $N$ 't Hooft limit, together with the unknown coefficient $c_{1}$, as follows:

$$
\begin{aligned}
\widetilde{W}(z) & =\left(d ^ { a b c d } \left[J^{a} J^{b} J^{c} J^{d}(z)+\frac{4 \lambda(5 \lambda+4)}{3(\lambda-1)(\lambda+2)} J^{a} J^{b} J^{c} K^{d}(z)+\frac{2 \lambda^{2}(5 \lambda+7)}{(\lambda-1)^{2}(\lambda+2)} J^{a} J^{b} K^{c} K^{d}(z)\right.\right. \\
& \left.+\frac{20 \lambda^{2}(\lambda+1)}{3(\lambda-3)(\lambda-1)(\lambda+2)} J^{a} K^{b} K^{c} K^{d}(z)\right]+d^{a b e} d^{c d e}\left[\frac{2 \lambda}{\lambda+2} J^{a} J^{b} J^{c} J^{d}(z)\right. \\
& +\frac{8 \lambda}{(\lambda-1)(\lambda+2)} J^{a} J^{b} J^{c} K^{d}(z)+\frac{12 \lambda^{2}(\lambda+3)}{(\lambda-2)(\lambda-1)^{2}(\lambda+2)} J^{a} J^{b} K^{c} K^{d}(z) \\
& \left.+\frac{40 \lambda^{2}(\lambda+1)}{(\lambda-3)(\lambda-2)(\lambda-1)(\lambda+2)} J^{a} K^{b} K^{c} K^{d}(z)\right]-N^{2}\left[\frac{4 \lambda}{3(\lambda+2)} \partial^{2} J^{a} K^{a}(z)\right. \\
& \left.\left.-\frac{4 \lambda(\lambda+1)}{(\lambda-1)(\lambda+2)} \partial J^{a} \partial K^{a}(z)+\frac{4 \lambda(\lambda+1)}{3(\lambda-1)(\lambda+2)} J^{a} \partial^{2} K^{a}(z)\right]\right) c_{1} .
\end{aligned}
$$

At first sight, the last three terms become very large compared to the rest but this is not true. We will see that the $N$ dependence of each term behaves equally. Let us focus on the zero mode of spin 4 current. By realizing the equation (3.17), one can reexpress the zero mode as follows:

$$
\begin{aligned}
d^{a b c d} J_{0}^{a} J_{0}^{b} J_{0}^{c} J_{0}^{d} & \rightarrow 3 d^{a b e} d^{c d e} J_{0}^{a} J_{0}^{b} J_{0}^{c} J_{0}^{d}-3 N^{2}\left(\partial J^{a}\right)_{0}\left(\partial J^{a}\right)_{0}+2 N^{2}\left(\partial^{2} J^{a}\right)_{0}\left(J^{a}\right)_{0} \\
& =3 d^{a b e} d^{c d e} J_{0}^{a} J_{0}^{b} J_{0}^{c} J_{0}^{d}+N^{2} J_{0}^{a} J_{0}^{a},
\end{aligned}
$$

where the Laurent mode expansion is used 8. The contribution from $J_{0}^{a} J_{0}^{a} J_{0}^{b} J_{0}^{b}$ is removed under (3.19) because the behavior of this term in (3.17) depends on $N=\frac{1}{N} \times N^{2}$. The zero mode of (3.20) can be written as

$$
\begin{aligned}
\widetilde{W}_{0} & =\left[-\frac{\left(18+15 \lambda+111 \lambda^{2}-7 \lambda^{3}+7 \lambda^{4}\right)}{3(\lambda-3)(\lambda-1)^{2}(\lambda+2)} d^{a b c d} J_{0}^{a} J_{0}^{b} J_{0}^{c} J_{0}^{d}\right. \\
& +\frac{2 \lambda\left(30-95 \lambda+41 \lambda^{2}-25 \lambda^{3}+\lambda^{4}\right)}{(\lambda-3)(\lambda-2)(\lambda-1)^{2}(\lambda+2)} d^{a b e} d^{c d e} J_{0}^{a} J_{0}^{b} J_{0}^{c} J_{0}^{d} \\
& \left.+N^{2} \frac{4 \lambda(\lambda-3)}{3(\lambda-1)(\lambda+2)} J_{0}^{a} J_{0}^{a}\right] c_{1},
\end{aligned}
$$

8 That is, $J^{a}(z)=\sum_{m \in \mathbf{Z}} \frac{J_{m}^{a}}{z^{m+1}}, \partial J^{a}(z)=-\sum_{m \in \mathbf{Z}}(m+1) \frac{J_{m}^{a}}{z^{m+2}}$ and $\partial^{2} J^{a}(z)=\sum_{m \in \mathbf{Z}}(m+1)(m+2) \frac{J_{m}^{a}}{z^{m+3}}$. 
where the singlet condition $K_{0}^{a}=-J_{0}^{a}$ is used. Note that, in [1], the second element of highest weight representations corresponds to the diagonal current $J^{\prime a}(z)=J^{a}(z)+K^{a}(z)$ and the first element corresponds to the current $K^{a}(z)$. We are looking for the eigenvalue equation of (3.22) acting on the representation $(f ; 0) \otimes(f ; 0)$ where $J_{0}^{a}+K_{0}^{a}=0$. The first term in (3.22) is the sum of the first four terms in (3.20) with alternative signs. Similarly, the second term in (3.22) is the sum of the next four terms in (3.20) with alternative signs and finally, the third term in (3.22) is the sum of the last three terms in (3.20) with appropriate signs and multiplicities.

One should compute the spin 4 zero mode (3.22) on the fundamental representation. The product of $S U(N)$ generators we are using in this paper has the following decompositions with $\delta, d$ and $f$ symbols

$$
T^{a} T^{b}=-\frac{1}{N} \delta^{a b}-\frac{i}{2} d^{a b c} T^{c}+\frac{1}{2} f^{a b c} T^{c} .
$$

From the relation (3.23), one obtains the quartic product with two $d$ symbols. Among nine terms, the nonzero five terms (due to the traceless condition for antihermitian basis) can be obtained and using the property (A.3), one finds

$$
\begin{aligned}
d^{a b e} d^{c d e} \operatorname{Tr}\left(T^{a} T^{b} T^{c} T^{d}\right) & =\frac{1}{4} d^{a b e} d^{c d e} d^{a b f} d^{c d f}=\frac{1}{4} \frac{2}{N}\left(N^{2}-4\right) \frac{2}{N}\left(N^{2}-4\right) \delta^{a a} \\
& =\frac{1}{N^{2}}\left(N^{2}-4\right)^{2}\left(N^{2}-1\right),
\end{aligned}
$$

where the property (A.4) is used. Since we want to obtain the eigenvalue not the trace as in (3.24), we should divide this by $N$. Then, under (3.19), one arrives at the zero mode that appears in the second term in (3.22) acting on the fundamental representation,

$$
d^{a b e} d^{c d e} J_{0}^{a} J_{0}^{b} J_{0}^{c} J_{0}^{d}\left|f>=N^{3}\right| f>.
$$

Similarly, the zero mode that appears in the last term in (3.22) acts on the fundamental representation,

$$
\delta^{a b} \operatorname{Tr}\left(T^{a} T^{b}\right)=-\delta^{a a}=-\left(N^{2}-1\right) \rightarrow J_{0}^{a} J_{0}^{a}|f>=-N| f>.
$$

One also has the same expressions (3.25) and (3.26) on the antifundamental representation.

By using the equations (3.21), (3.25) and (3.26), one simplifies the right hand side of (3.22) and surprisingly, it turns out that it is very simple factorized form

$$
\begin{aligned}
& N^{3}\left[-\frac{\left(18+15 \lambda+111 \lambda^{2}-7 \lambda^{3}+7 \lambda^{4}\right)}{3(\lambda-3)(\lambda-1)^{2}(\lambda+2)}(3-1)+\frac{2 \lambda\left(30-95 \lambda+41 \lambda^{2}-25 \lambda^{3}+\lambda^{4}\right)}{(\lambda-3)(\lambda-2)(\lambda-1)^{2}(\lambda+2)}\right. \\
& \left.-\frac{4 \lambda(\lambda-3)}{3(\lambda-1)(\lambda+2)}\right] c_{1}=N^{3}\left[\frac{4(1+\lambda)^{2}(3+\lambda)}{(3-\lambda)(2-\lambda)(1-\lambda)}\right] c_{1} .
\end{aligned}
$$


For the primary $(f ; 0) \otimes(f ; 0)$ where $J_{0}^{a}+K_{0}^{a}=0$, from the result of (3.27), one can compute

$$
\widetilde{W}_{0}\left|\mathcal{O}_{+}>=N^{3}\left[\frac{4(1+\lambda)^{2}(3+\lambda)}{(3-\lambda)(2-\lambda)(1-\lambda)}\right] c_{1}\right| \mathcal{O}_{+}>, \quad \mathcal{O}_{+} \equiv(f ; 0) \otimes(f ; 0)
$$

and for the primary $(0 ; f) \otimes(0 ; f)$ where $K_{0}^{a}=0$, the spin 4 current acts as

$$
\widetilde{W}_{0}\left|\mathcal{O}_{-}>=N^{3}\left[\frac{4(1+\lambda)}{(2+\lambda)}\right] c_{1}\right| \mathcal{O}_{-}>, \quad \mathcal{O}_{-} \equiv(0 ; f) \otimes(0 ; f),
$$

where one uses $\left[3-1+\frac{2 \lambda}{\lambda+2}\right] N^{3}=N^{3}\left[\frac{4(1+\lambda)}{(2+\lambda)}\right] c_{1}$ that corresponds to the equation (3.22) by choosing the first and fifth terms in (3.20). For the other primaries $\overline{\mathcal{O}}_{+} \equiv(\bar{f} ; 0) \otimes(\bar{f} ; 0)$ and $\overline{\mathcal{O}}_{-} \equiv(0 ; \bar{f}) \otimes(0 ; \bar{f})$, one has similar relations. The generators in the antifundamental representation has an extra minus sign, compared to the generators in the fundamental representation, but this does not affect the relations (3.24) and (3.26) because the number of the power of $T^{a}$ is even. Then we have same equations with (3.28) and (3.29) by replacing $\mid \mathcal{O}_{ \pm}>$ with $\mid \overline{\mathcal{O}}_{ \pm}>$respectively. There are no sign changes in the eigenvalues, contrary to the spin 3 case [16, 11] where the number of the power of $T^{a}$ is odd. According to the normalization of [11], the eigenvalues of the spin 4 zero mode acting on the above primaries appear as follows:

$$
\begin{aligned}
& U_{0}^{4}\left|\mathcal{O}_{+}>=(1+\lambda)(2+\lambda)(3+\lambda)\right| \mathcal{O}_{+}>, \quad \mathcal{O}_{+} \equiv(f ; 0) \otimes(f ; 0), \\
& U_{0}^{4}\left|\mathcal{O}_{-}>=(1-\lambda)(2-\lambda)(3-\lambda)\right| \mathcal{O}_{-}>, \quad \mathcal{O}_{-} \equiv(0 ; f) \otimes(0 ; f) \text {. }
\end{aligned}
$$

The different normalization in [16] is used. For given relations (3.28) and (3.29), it is possible to determine the unknown coefficient function $c_{1}(N, k)$ in order to satisfy the condition (3.30) as follows:

$$
c_{1}(N, \lambda)=\frac{(1-\lambda)(2-\lambda)(3-\lambda)}{\left[N^{3} \frac{4(1+\lambda)}{(2+\lambda)}\right]} .
$$

This relation (3.31) is a generalization of (4.24) of [16] for the higher spin greater than 3 . According to (3.25), the factor $N^{3}$ comes from the quartic Casimir for the fundamental representation. For the spin 4 case in our paper, the eigenvalue equation has other factor from (3.29). In other words, the $\widetilde{W}(z)$ in the large $N$ limit has $\lambda$ dependent term in $c_{6}$ coefficient: $\frac{2 \lambda}{\lambda+2}$ from (F.10). Combined with the constant piece, the overall factor depends on the 't Hooft coupling constant $\lambda$ explicitly. Therefore, we have

$$
\begin{aligned}
& \widetilde{W}_{0}\left|\mathcal{O}_{+}>=(1+\lambda)(2+\lambda)(3+\lambda)\right| \mathcal{O}_{+}>, \quad \mathcal{O}_{+} \equiv(f ; 0) \otimes(f ; 0), \\
& \widetilde{W}_{0}\left|\mathcal{O}_{-}>=(1-\lambda)(2-\lambda)(3-\lambda)\right| \mathcal{O}_{-}>, \quad \mathcal{O}_{-} \equiv(0 ; f) \otimes(0 ; f) \text {. }
\end{aligned}
$$


In next subsection, we would like to look for more general coset spin 4 field which satisfies the above condition (3.30).

Following the analysis by Chang and Yin [14], from the action of $\widetilde{W}_{0}$ on the primary states, (3.32), the three-point function with scalars is summarized as

$$
\begin{array}{ll}
<\overline{\mathcal{O}}_{+} \mathcal{O}_{+} \widetilde{W}>=(1+\lambda)(2+\lambda)(3+\lambda), & \overline{\mathcal{O}}_{+} \equiv(\bar{f} ; 0) \otimes(\bar{f} ; 0) \\
<\overline{\mathcal{O}}_{-} \mathcal{O}_{-} \widetilde{W}>=(1-\lambda)(2-\lambda)(3-\lambda), & \overline{\mathcal{O}}_{-} \equiv(0 ; \bar{f}) \otimes(0 ; \bar{f}) .
\end{array}
$$

Note that in [14], the normalization for the spin 3 current is the same as that in [16] 9. As a spin increases, the extra $\lambda$ dependent factors occur in the three-point functions.

How does one compare the three-point function (3.33) to that from the bulk computation in [14]? With the normalization $\left\langle J^{(s)}(z) J^{(s)}(w)>=1\right.$, the three-point function at $\lambda=\frac{1}{2}$ is given by

$$
<\overline{\mathcal{O}}_{+} \mathcal{O}_{+} J^{(s)}>=N^{-\frac{1}{2}} \Gamma(s) \sqrt{\frac{2 s-1}{\Gamma(2 s-1)}}, \quad<\overline{\mathcal{O}}_{-} \mathcal{O}_{-} J^{(s)}>=(-1)^{s} N^{-\frac{1}{2}} \frac{\Gamma(s)}{\sqrt{\Gamma(2 s)}}
$$

In our case, we did not compute the operator product expansion $\widetilde{W}(z) \widetilde{W}(w)$ explicitly where $\widetilde{W}(z)$ is given by (3.20). We will have $<\widetilde{W}(z) \widetilde{W}(w)>=A(N, k)+\left(\frac{1}{N}\right.$ correction) by computing the highest singular terms $\frac{1}{(z-w)^{8}}$. Let us divide the two relations (3.33). Then we do not have to worry about the normalization for $\widetilde{W}(z)$.

$$
\frac{<\overline{\mathcal{O}}_{+} \mathcal{O}_{+} \widetilde{W}>}{<\overline{\mathcal{O}}_{-} \mathcal{O}_{-} \widetilde{W}>}=\left.\frac{(1+\lambda)(2+\lambda)(3+\lambda)}{(1-\lambda)(2-\lambda)(3-\lambda)} \rightarrow\left(\frac{3+\lambda}{1-\lambda}\right)\right|_{\lambda=\frac{1}{2}}=7
$$

where we put $\lambda=\frac{1}{2}$ at the final stage. Note that the factor $(1+\lambda)(2+\lambda)$ in the numerator cancels the factor $(2-\lambda)(3-\lambda)$ in the denominator at $\lambda=\frac{1}{2}$. On the other hand, by taking the ratio in (3.34), one obtains

$$
\frac{<\overline{\mathcal{O}}_{+} \mathcal{O}_{+} J^{(s)}>}{<\overline{\mathcal{O}}_{-} \mathcal{O}_{-} J^{(s)}>}=(-1)^{s}(2 s-1) .
$$

For $s=4$, this relation (3.36) is exactly the same as the relation (3.35) 10 . One can continue to analyze for higher spin greater than 4 . From $<\overline{\mathcal{O}}_{+} \mathcal{O}_{+} \widetilde{W}^{(s)}>=(-1)^{s}(1+\lambda)(2+\lambda)(3+$

\footnotetext{
${ }^{9}$ In the notation of [11], the three-point function for the spin 3 current is $\left.<\overline{\mathcal{O}}_{+} \mathcal{O}_{+} \widetilde{T}^{(3)}\right\rangle=-(1+\lambda)(2+\lambda)$ and $\left\langle\overline{\mathcal{O}} \_\mathcal{O}_{-} \widetilde{T}^{(3)}\right\rangle=(1-\lambda)(2-\lambda)$ corresponding to the equation $(5.14)$ of [14].

10 This feature occurs for $s=2,3$ case. For $s=3$, the ratio $\frac{\left\langle\overline{\mathcal{O}}_{+} \mathcal{O}_{+} \widetilde{T}^{(3)}\right\rangle}{\left\langle\overline{\mathcal{O}}_{-} \mathcal{O}_{-} \widetilde{T}^{(3)}\right\rangle}=-\frac{(1+\lambda)(2+\lambda)}{(1-\lambda)(2-\lambda)} \rightarrow-\left.\left(\frac{2+\lambda}{1-\lambda}\right)\right|_{\lambda=\frac{1}{2}}=$ -5 which is the same as (13.36) for $s=3$. Similarly, for $s=2$, we have $\frac{\left\langle\overline{\mathcal{O}}_{+} \mathcal{O}_{+} \widetilde{T}^{(2)}\right\rangle}{\left\langle\overline{\mathcal{O}}_{-} \mathcal{O}_{-} \widetilde{T}^{(2)}\right\rangle}=\left.\frac{\frac{1}{2}(1+\lambda)}{\frac{1}{2}(1-\lambda)} \rightarrow\left(\frac{1+\lambda}{1-\lambda}\right)\right|_{\lambda=\frac{1}{2}}=$ 3 which coincides with (3.36) for $s=2$.
} 
$\lambda) \cdots(s-1+\lambda)$ and $<\overline{\mathcal{O}}_{-} \mathcal{O}_{-} \widetilde{W}^{(s)}>=(1-\lambda)(2-\lambda)(3-\lambda) \cdots(s-1-\lambda)$, one takes the ratio and realizes that the factors in the numerator cancel the factors in the denominator at $\lambda=\frac{1}{2}$,

$$
\begin{aligned}
\frac{\left\langle\overline{\mathcal{O}}_{+} \mathcal{O}_{+} \widetilde{W}^{(s)}>\right.}{<\overline{\mathcal{O}}_{-} \mathcal{O}_{-} \widetilde{W}^{(s)}>} & =\frac{(-1)^{s}(1+\lambda)(2+\lambda)(3+\lambda) \cdots(s-1+\lambda)}{(1-\lambda)(2-\lambda)(3-\lambda) \cdots(s-1-\lambda)} \\
& \left.\rightarrow(-1)^{s}\left(\frac{s-1+\lambda}{1-\lambda}\right)\right|_{\lambda=\frac{1}{2}}=(-1)^{s}(2 s-1)
\end{aligned}
$$

This relation (3.37) is the same as the relation (3.36). It would be interesting to construct the three-point functions in the deformed $A d S_{3}$ bulk theory, as mentioned in [14], that generalize to the equations (3.34) and compare to the three-point functions (3.33) for all values of 't Hooft coupling constant in the $W_{N}$ coset conformal field theory in the large $N$ 't Hooft limit.

\subsection{The general coset primary spin-4 current $\widetilde{W}^{(4)}(z)$ and its large $N$ 't Hooft limit}

More generally, one can consider the coset spin 4 field that contains the field $\widetilde{T}^{2}(z)$ given by (G.6). Or one can understand that according to the second equation of (G.2), if we write down the field $d^{a b c d} J^{a} J^{b} K^{c} K^{d}(z)$ using the identity (2.12), then there exists an independent field $J^{a} J^{b} K^{a} K^{b}(z)$ appears naturally. The only new term from the relation (G.6), compared to the relation (3.16) , is $J^{a} J^{b} K^{a} K^{b}(z)$ and let us add this term with the coefficient function $c_{23}$ to the previous coset spin 4 field (3.16):

$$
\widetilde{W}^{(4)}(z)=\widetilde{W}(z)+c_{23} J^{a} J^{b} K^{a} K^{b}(z) .
$$

Then one should have the above two properties (3.11) and (3.12) for the new field (3.38). That is, $J^{\prime a}(z) \widetilde{W}^{(4)}(w)=$ regular and $\widetilde{W}^{(4)}(z)$ is a primary field of dimension 4 under the

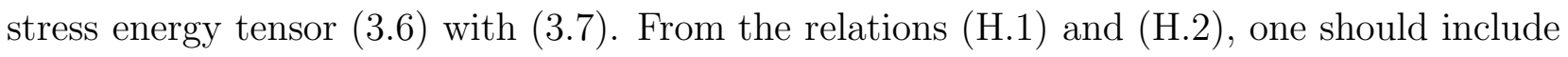
$c_{23}$ dependent terms into $(\underline{\mathrm{F} .1})-(\underline{\mathrm{F} .7})$. It turns out that the more general spin 4 coset field is

$$
\begin{aligned}
\widetilde{W}^{(4)}(z) & =d^{a b c d}\left[c_{1} J^{a} J^{b} J^{c} J^{d}+\left(c_{2}+b_{2}\right) J^{a} J^{b} J^{c} K^{d}+\left(c_{3}+b_{3}\right) J^{a} J^{b} K^{c} K^{d}+\left(c_{4}+b_{4}\right) J^{a} K^{b} K^{c} K^{d}\right. \\
& \left.+\left(c_{5}+b_{5}\right) K^{a} K^{b} K^{c} K^{d}\right]+d^{a b e} d^{c d e}\left[\left(c_{6}+b_{6}\right) J^{a} J^{b} J^{c} J^{d}+\left(c_{7}+b_{7}\right) J^{a} J^{b} J^{c} K^{d}\right. \\
& \left.+\left(c_{8}+b_{8}\right) J^{a} J^{b} K^{c} K^{d}+\left(c_{9}+b_{9}\right) J^{a} K^{b} K^{c} K^{d}+\left(c_{10}+b_{10}\right) K^{a} K^{b} K^{c} K^{d}\right] \\
& +\left(c_{11}+b_{11}\right) J^{a} J^{a} J^{b} J^{b}+\left(c_{12}+b_{12}\right) J^{a} J^{a} J^{b} K^{b}+\left(c_{13}+b_{13}\right) J^{a} J^{a} K^{b} K^{b} \\
& +\left(c_{14}+b_{14}\right) J^{a} K^{a} K^{b} K^{b}+\left(c_{15}+b_{15}\right) K^{a} K^{a} K^{b} K^{b}+\left(c_{17}+b_{17}\right) \partial^{2} J^{a} K^{a} \\
& +\left(c_{20}+b_{20}\right) \partial J^{a} \partial K^{a}+\left(c_{22}+b_{22}\right) J^{a} \partial^{2} K^{a}+c_{23} J^{a} J^{b} K^{a} K^{b},
\end{aligned}
$$


where the coefficients $c_{i}$ are given by the equations (F.8) and the coefficients $b_{i}$ are given by the equations ( $(\underline{H .3})$. In order to see how the modification arises, we keep the old coefficient functions $c_{i}$ and present the extra terms characterized by the coefficients $b_{i}$. The two unknown coefficient functions $c_{1}$ and $c_{23}$ cannot be fixed by the above requirements.

In Appendix I, we have found the coset spin 4 field discussed in [5] explicitly. From the beginning, the coset spin 3 field, $\widetilde{T}^{(3)}$, is completely determined by the regularity condition (3.11) and the condition for the primary field corresponding to (3.12) except the overall factor which is fixed by the highest singular term, in the operator product expansion $\widetilde{T}^{(3)}(z) \widetilde{T}^{(3)}(w)$, that behaves as $\frac{1}{3} \widetilde{c}$ with the relation (3.9). By focusing on the $\frac{1}{(z-w)^{2}}$ terms in the above operator product expansion, one obtains a new primary coset spin 4 field explicitly (this is not known so far) and it is given by the equations (I.3), (I.4), $\widetilde{\Lambda}(w)$ and $\partial^{2} \widetilde{T}(w)$. Alternatively, it is easy to see that this new primary field $\widetilde{R}^{(4)}(z)$ in the Appendix $I$ is nothing but the $\widetilde{W}^{(4)}(z)$ in (3.39) with fixed $c_{1}$ and $c_{23}$ that are given on (1.9) and (1.10). This is summarized in the relation (I.11). Note that the field $J^{a} J^{b} K^{a} K^{b}(z)$ arises in the equation (I.3) very naturally. That is, it comes from the $d_{23}$ term and $\widetilde{T}^{2}(w)$ term.

In the large $N$ limit (3.19), the term proportional to $d^{a b c d} K^{a} K^{b} K^{c} K^{d}(z)$ behaves as $\frac{1}{N}$ and goes to zero. Similarly, the term with $d^{\text {abe }} d^{\text {cde }} K^{a} K^{b} K^{c} K^{d}(z)$ behaves as $\frac{1}{N}$ also and vanishes. The terms with $b_{13}, b_{14}$ have $\frac{1}{N}$ dependence and the term with $b_{15}$ has $\frac{1}{N^{2}}$ dependence. See the equations ( $(\mathbf{H . 4})$ for details. Then, all of these vanish in the large $N$ limit. The large $N$ 't Hooft limit of (3.20) is generalized to the following expression, from (․․ㄱ $)$ and large $N$ limits of $\widetilde{\Lambda}(z)$ and $\partial^{2} \widetilde{T}(z)$, as follows:

$$
\begin{aligned}
\widetilde{W}^{(4)}(z) & =\widetilde{W}(z)+\left(d ^ { a b c d } \left[-\frac{(-1+\lambda)(1+\lambda)}{2 \lambda(2+\lambda)} J^{a} J^{b} J^{c} K^{d}(z)-\frac{3(1+\lambda)}{8(2+\lambda)} J^{a} J^{b} K^{c} K^{d}(z)\right.\right. \\
& \left.-\frac{5(-1+\lambda)(1+\lambda)}{12(-3+\lambda)(2+\lambda)} J^{a} K^{b} K^{c} K^{d}(z)\right]+d^{a b e} d^{c d e}\left[-\frac{(-1+\lambda)^{2}(1+\lambda)}{4 \lambda^{2}(2+\lambda)} J^{a} J^{b} J^{c} J^{d}(z)\right. \\
& +\frac{(-1+\lambda)(1+\lambda)}{2 \lambda(2+\lambda)} J^{a} J^{b} J^{c} K^{d}(z)-\frac{(1+\lambda)(1+2 \lambda)}{4(-2+\lambda)(2+\lambda)} J^{a} J^{b} K^{c} K^{d}(z) \\
& \left.-\frac{5(-1+\lambda)(1+\lambda)}{2(-3+\lambda)(-2+\lambda)(2+\lambda)} J^{a} K^{b} K^{c} K^{d}(z)\right]+\frac{(-1+\lambda)^{2}}{4 \lambda^{2}} J^{a} J^{a} J^{b} J^{b}(z) \\
& +\frac{(-1+\lambda)}{\lambda} J^{a} J^{a} J^{b} K^{b}(z)+J^{a} J^{b} K^{a} K^{b}(z)+N^{2}\left[\frac{(-1+\lambda)(1+\lambda)}{4 \lambda(2+\lambda)} \partial^{2} J^{a} K^{a}(z)\right. \\
& \left.\left.-\frac{(-1+\lambda)(1+\lambda)}{4 \lambda(2+\lambda)} \partial J^{a} \partial K^{a}(z)+\frac{(-1+\lambda)(1+\lambda)}{12 \lambda(2+\lambda)} J^{a} \partial^{2} K^{a}(z)\right]\right) c_{23} .
\end{aligned}
$$

One can compute the contribution from $J^{a} J^{b} K^{a} K^{b}(z)$ by realizing that the trace $\operatorname{Tr}\left(T^{a} T^{b} T^{a} T^{b}\right)$ with (3.23) can be calculated. There is no $N^{2}$ term and the next leading term behaves as $\frac{1}{N}$. In other words, the field $J^{a} J^{b} K^{a} K^{b}(z)$ itself does not contribute to the final answer, but 
its presence does affect all the other coefficient functions. The terms with $c_{23}$ in the relation (3.40) also appear in the relation (3.20). Therefore, in (3.40), the independent terms are the same as the one in (3.20) with different coefficient functions.

By taking the same analysis in the previous subsection 3.3 (i.e., (3.25) and (3.26)), the eigenvalue (3.28) acting on $(f ; 0) \otimes(f ; 0)$ is generalized to

$$
\widetilde{W}_{0}^{(4)}\left|\mathcal{O}_{+}>=N^{3} \frac{(1+\lambda)^{2}(3+\lambda)}{(1-\lambda)(2-\lambda)(3-\lambda)}\left[4 c_{1}-\frac{(1-\lambda)^{2}}{4 \lambda^{2}} c_{23}\right]\right| \mathcal{O}_{+}>\text {. }
$$

Similarly, one has the following generalized expression on $(0 ; f) \otimes(0 ; f)$

$$
\widetilde{W}_{0}^{(4)}\left|\mathcal{O}_{-}>=N^{3} \frac{(1+\lambda)}{(2+\lambda)}\left[4 c_{1}-\frac{(1-\lambda)^{2}}{4 \lambda^{2}} c_{23}\right]\right| \mathcal{O}_{-}>
$$

By choosing the two undetermined coefficients $c_{1}(N, k)$ and $c_{23}(N, k)$,

$$
c_{1}(N, \lambda)=0, \quad c_{23}(N, \lambda)=-\frac{(1-\lambda)(2-\lambda)(3-\lambda)}{\left[N^{3} \frac{(1-\lambda)^{2}(1+\lambda)}{4 \lambda^{2}(2+\lambda)}\right]},
$$

and plugging (3.43) into (3.41) and (3.42) one has the following relations,

$$
\begin{aligned}
& \widetilde{W}_{0}^{(4)}\left|\mathcal{O}_{+}>=(1+\lambda)(2+\lambda)(3+\lambda)\right| \mathcal{O}_{+}> \\
& \widetilde{W}_{0}^{(4)}\left|\mathcal{O}_{-}>=(1-\lambda)(2-\lambda)(3-\lambda)\right| \mathcal{O}_{-}>
\end{aligned}
$$

which are exactly the same as the equations (3.30). In this case, there is no $d^{a b c d} J^{a} J^{b} J^{c} J^{d}(z)$ term because $c_{1}(N, \lambda)=0$. The similar relations hold for $\mid \overline{\mathcal{O}}_{ \pm}>$as we explained before.

More generally, as long as the following relation,

$$
4 c_{1}(N, \lambda)-\frac{(1-\lambda)^{2}}{4 \lambda^{2}} c_{23}(N, \lambda)=\frac{(1-\lambda)(2-\lambda)(3-\lambda)}{\left[N^{3} \frac{(1+\lambda)}{(2+\lambda)}\right]}
$$

holds, the above equation (3.44) is satisfied. The case with (3.31) and the case with (3.43) are the particular solutions of the relation (3.45). Recall that the denominator in (3.45) is exactly the same as the overall factor on the right hand side of (3.42) that comes from the $d^{a b e} d^{c d e} J^{a} J^{b} J^{c} J^{d}(z)$ and other terms in $\widetilde{W}(z)$ we have discussed before. It seems that this behavior can be generalized to the higher spin greater than 4 and the right hand side of (3.42) for $\widetilde{W}_{0}^{(s)}$ should behave as $\prod_{i=1}^{s-1}(i-\lambda)$ where $s$ is a spin. Then the right hand side of (3.41) for $\widetilde{W}^{(s)}$ can be fixed automatically.

For the field $\widetilde{W}^{(4)}(z)$, the only requirements we used so far are given in 1) and 2) in the introduction and it turns out that there are two undetermined coefficients with the constraint (3.45). This implies that these requirements are not enough to fix them completely. Are there 
any ways to determine these two undetermined coefficients? Recall that the field contents of $W_{N}$ minimal model are given by the spin-2 field, the spin-3 field, the spin- 4 field and so on 11. The operator product expansion of primary spin-3 field with itself should provide further constraint on the above spin-4 field.

From the result of the Appendix I, one can think of the following spin 4 primary field

$$
\alpha(N, k) \widetilde{R}^{(4)}(z)
$$

where $\widetilde{R}^{(4)}(z)$ is the spin-4 primary field described in [5] or in the Appendix $I$ and $\alpha(N, k)$ is an arbitrary constant which depends on $N, k$. One takes the large $N$ 't Hooft limit for (I.9) and ([.10) (or ([1.12) ) and then using the equation (3.40) or the equations (3.41) and (3.42), one obtains

$$
\begin{aligned}
& \widetilde{R}_{0}^{(4)}\left|\mathcal{O}_{+}>=\frac{2(1+\lambda)(3+\lambda)}{5(2-\lambda)}\right| \mathcal{O}_{+}> \\
& \widetilde{R}_{0}^{(4)}\left|\mathcal{O}_{-}>=\frac{2(1-\lambda)(3-\lambda)}{5(2+\lambda)}\right| \mathcal{O}_{-}>
\end{aligned}
$$

In this case, the eigenvalues are very symmetric in the 't Hooft coupling $\lambda$. The first equation in (3.47) goes to the second equation in (3.47) by taking $\lambda \rightarrow-\lambda$ and vice versa. Then it is straightforward to compute the three-point functions from the relations (3.41) and (3.42) or the equation (3.47). For $\alpha(N, k)=\frac{5}{2}(2-\lambda)(2+\lambda)$, one has the same equations as (3.44) for the zero mode of (3.46). One can easily see that the $c_{1}$ and $c_{23}$ in (I.12) multiplied by the $\alpha(N, k)$ satisfy the above relation (3.45). This is an expected result from the identity (I.11). Note that the field $\widetilde{R}^{(4)}(z)$ is obtained from the operator product expansion of two spin-3 fields and all the coefficient functions are known. Once the highest singular term $\frac{1}{(z-w)^{8}}$ in the operator product expansion of spin-4 field with itself, in the large $N$, is computed, then one can normalize such that the two-point function is equal to 1 and this will fix the overall constant completely.

\section{Conclusions and outlook}

We have found the coset primary spin-4 field $\widetilde{W}^{(4)}(z)$ in (3.39) together with the coefficient functions ( $(\underline{\mathrm{F} .8}),(\underline{\mathrm{F} .9})$ and $(\underline{\mathrm{H} .3})$ where the two coefficient functions $c_{1}$ and $c_{23}$ are given by

\footnotetext{
11 The vacuum character, the equation (7.18) of [10] or the equation (2.18) of [1], is given by $\frac{1}{\prod_{s=2}^{N} \prod_{n=s}^{\infty}\left(1-q^{n}\right)}=1+q^{2}+2 q^{3}+4 q^{4}+6 q^{5}+12 q^{6}+\mathcal{O}\left(q^{7}\right)$. The 4 in front of $q^{4}$ indicates that there are four spin-4 fields, $\widetilde{T}^{2}(z), \partial^{2} \widetilde{T}(z), \partial \widetilde{T}^{(3)}(z)$ and $\widetilde{W}^{(4)}(z)$. Among these four fields, the only primary field is $\widetilde{W}^{(4)}(z)$ up to normalization. We thank the referee for pointing out this observation.
} 
the equations (1.9). The large $N$ limit of (3.39) satisfies the zero mode acting on the primary states (3.44). Furthermore, the three-point functions for $\widetilde{W}^{(4)}(z)$ with the scalars take the form (3.33) and they are dual to the three-point functions in [14].

- How does one perform the operator algebra consisting of spins-2,3 and 4? Among the possible six operator product expansions, one of them is given by the operator product expansion (3.8). There are also two operator product expansions, (3.12) and similar operator product expansion for the spin 3 field $\widetilde{T}^{(3)}(z)$ (II.1). The operator product expansion $\widetilde{T}^{(3)}(z) \widetilde{T}^{(3)}(w)$ is known in [5]. Then there are two unknown operator product expansions $\widetilde{T}^{(3)}(z) \widetilde{W}^{(4)}(w)$ and $\widetilde{W}^{(4)}(z) \widetilde{W}^{(4)}(w)$. It would be interesting to find these and to see how they differ from the algebras found in [44, 45] although this work will be tedious. Furthermore, $\frac{1}{(z-w)^{3}}$ terms of the operator product expansion $\widetilde{W}^{(4)}(z) \widetilde{W}^{(4)}(w)$ or $\frac{1}{(z-w)^{2}}$ terms of the operator product expansion $\widetilde{T}^{(3)}(z) \widetilde{W}^{(4)}(w)$ will provide the structure of spin-5 field. See also the works in [46, 47, 48] for the operator product algebra between spins-2, 4 .

- It would be interesting to find the zero modes on all other representations. The question is how one can write down the generators for these higher order representations? For example, one can act the zero mode on the representation $(\mathrm{adj} ; 0)$ that appears in the fusion of $(f ; 0)$ and $(\bar{f} ; 0)$. In this case, the element of the generator $T^{a}$ in the adjoint representation for $S U(N)$ can be written as $\left(T^{a}\right)_{b c}=f^{a b c}$. Then the trace $\operatorname{Tr}\left(T^{a} T^{b} T^{c} T^{d}\right)$ is nothing but the quartic in $f$ symbols. Using the identity (A.12) in the Appendix $A$, one can simplify (3.24) as $2 N^{3}$ in the large $N$ limit where one should use the identity (A.8). Similarly, the equation (3.26) becomes $-2 N$. This reflects an overall extra factor 2 in this representation. This implies that one has $\widetilde{W}_{0}^{(4)}|(\operatorname{adj} ; 0) \otimes(\operatorname{adj} ; 0)>=2(1+\lambda)(2+\lambda)(3+\lambda)|(\operatorname{adj} ; 0) \otimes(\operatorname{adj} ; 0)>$ and $\widetilde{W}_{0}^{(4)}|(0 ; \operatorname{adj}) \otimes(0 ; \operatorname{adj})>=2(1-\lambda)(2-\lambda)(3-\lambda)|(0 ; \operatorname{adj}) \otimes(0 ; \operatorname{adj})>$. These can be seen from the equations (3.44) and their eigenvalue equations for $\mid \overline{\mathcal{O}}_{ \pm}>$. Since they have same eigenvalues (as we mentioned before), this is compatible with the above observation 12 .

- The coset primary spin-4 field is written in terms of WZW currents with finite $N, k$ dependent coefficient functions in the $W_{N}$ minimal model. How does this behavior appear in the higher spin gravity with matter in $A d S_{3}$ theory?

- It would be interesting to find the supersymmetric theory of [1] where there exist a field of dimension $\frac{3}{2}$ [49], a field of dimension $\frac{5}{2}$ [49, 50] and probably a field of dimension $\frac{7}{2}$. The first one and the stress energy tensor consists of the usual $\mathcal{N}=1$ super stress energy tensor, the second one and the dimension 3 primary field consists of $\mathcal{N}=1$ super field of dimension

\footnotetext{
12 This feature looks similar to the eigenvalue equations of spin-3 zero mode on the higher order representations $\left(\frac{N(N-1)}{2} ; 0\right)$ or $\left(0 ; \frac{N(N-1)}{2}\right)$. Recall that in spin-3 case, the zero mode eigenvalues of these states $(\operatorname{adj} ; 0)$ or $(0 ; \operatorname{adj})$ vanish due to the fact that the $d$ symbol of rank 3 contracted with three $f$ 's is equal to zero according to the identity (A.5).
} 
$\frac{5}{2}$ and the third one and the dimension 4 primary field gives another $\mathcal{N}=1$ super field of

dimension $\frac{7}{2}$. One way to see the field contents of dimension $\frac{7}{2}$ is to compute the operator product expansion of the field of dimension $\frac{5}{2}$ and a field of dimension 3 by focusing on $\frac{1}{(z-w)^{2}}$ terms.

- Can one generalize to the higher coset Casimir operator of spin greater than 4 and more generally of arbitrary spin $N$ ? As we described above, we should construct the completely symmetric traceless $d$ symbol of rank 5 which generalizes the $d$ symbol (2.12). The possible terms for $d$ symbol of rank 5 are $d^{a b c f} d^{f d e}$ or $d^{a b c} \delta^{d e}[42$.

- It would be interesting to find the coset spin-4 primary field in the other types of minimal models [50, 51]. In doing this, one should understand higher order invariant symmetric polynomials for $D_{\frac{N}{2}}, B_{\frac{N-1}{2}}$ that can be written in terms of the second lowest symmetric invariant polynomial for $D_{\frac{N}{2}}, B_{\frac{N-1}{2}}$ [42]. It will be useful to consider the coset model for fixed $N$ [52] in which there is a half integer spin as well as an integer spin.

- As described in the introduction, the eigenvalues of the zero modes of spins $2,3,4$, and 5 on the highest weight states of Fock space of $S U(N)$ Lie algebras were constructed in [39] using the quantum Miura transformation with Feigin-Fuchs type of free massless scalar fields. It would be interesting to find any relations between the findings in [39] and the results of [11] where the classical Miura transformation was used. The strategy for this classical limit 53 is as follows. At the classical level, any composite field term (product of $n$ fields) on the right hand side of operator product expansion should have its denominator proportional to $(n-1)$-th power of $c$. Therefore, as we take $c \rightarrow \infty$ in quantized operator product expansion, only those terms survive and any composite fields that do not satisfy this $c$-dependence will disappear in the classical limit.

\section{Acknowledgments}

We would like to thank the following people for correspondence on the following topics: C.-M. Chang on three point function, T. Hartman on the zero modes of degenerate representations, K. Thielemans on his mathematica package for operator product expansions, and G. Watts on higher spin generalization. This work was supported by the Mid-career Researcher Program through the National Research Foundation of Korea (NRF) grant funded by the Korean government (MEST) (No. 2009-0084601). CA acknowledges warm hospitality and partial support from the Department of Physics, Princeton University (I.R. Klebanov). 


\section{Appendix A Some properties of $f$ and $d$ tensors of $S U(N)$}

We list some properties between $d^{a b c}$ and $f^{a b c}$ symbols as follows:

$$
\begin{aligned}
& d^{a a b}=0, \\
& f^{a b c} f^{d b c}=2 N \delta^{a d}, \\
& f^{a b c} d^{d b c}=0 \text {, } \\
& d^{a b c} d^{d b c}=\frac{2}{N}\left(N^{2}-4\right) \delta^{a d}, \\
& f^{a d b} f^{b e c} f^{c f a}=-N f^{d e f}, \\
& d^{a d b} f^{b e c} f^{c f a}=-N d^{d e f}, \\
& d^{a d b} d^{b e c} f^{c f a}=\frac{\left(N^{2}-4\right)}{N} f^{d e f}, \\
& d^{a d b} d^{b e c} d^{c f a}=\frac{\left(N^{2}-12\right)}{N} d^{d e f}, \\
& f^{a d e} f^{e b c}+f^{b d e} f^{e c a}+f^{c d e} f^{e a b}=0, \\
& f^{a d e} d^{e b c}+f^{b d e} d^{e c a}+f^{c d e} d^{e a b}=0, \\
& f^{a b c} f^{c d e}=-\frac{4}{N}\left(\delta^{a e} \delta^{b d}-\delta^{a d} \delta^{b e}\right)-\left(d^{b d c} d^{c a e}-d^{a d c} d^{c b e}\right), \\
& f^{h a e} f^{e b f} f^{f c g} f^{g d h}=4 \delta^{a b} \delta^{c d}+4 \delta^{a d} \delta^{b c} \\
& +\frac{N}{2}\left(d^{a b e} d^{c d e}+d^{a d e} d^{e b c}-d^{a c e} d^{b d e}\right), \\
& f^{h a e} f^{e b f} f^{f c g} d^{g d h}=-\frac{N}{2} d^{a b e} f^{c d e}-\frac{N}{2} f^{a b e} d^{c d e}, \\
& f^{h a e} f^{e b f} d^{f c g} d^{g d h}=\frac{4\left(4-N^{2}\right)}{N^{2}}\left(\delta^{a b} \delta^{c d}-\delta^{a c} \delta^{b d}\right) \\
& +\frac{\left(8-N^{2}\right)}{2 N}\left(d^{a b e} d^{c d e}-d^{a c e} d^{b d e}\right)-\frac{N}{2} d^{a d e} d^{b c e}, \\
& f^{h a e} d^{e b f} f^{f c g} d^{g d h}=\frac{N}{2}\left(d^{a c e} d^{b d e}-d^{a d e} d^{b c e}\right)-\frac{N}{2} d^{a b e} d^{c d e}, \\
& f^{h a e} d^{e b f} d^{f c g} d^{g d h}=\frac{\left(N^{2}-12\right)}{2 N} f^{a b e} d^{c d e}+\frac{N}{2} d^{a b e} f^{c d e} \\
& +\frac{2}{N}\left(f^{a d e} d^{b c e}-f^{a c e} d^{b d e}\right), \\
& d^{\text {hae }} d^{e b f} d^{f c g} d^{g d h}=\frac{4\left(N^{2}-4\right)}{N^{2}}\left(\delta^{a b} \delta^{c d}+\delta^{a d} \delta^{b c}\right)-\frac{N}{2} d^{a c e} d^{b d e} \\
& +\frac{\left(N^{2}-16\right)}{2 N}\left(d^{a b e} d^{c d e}+d^{a d e} d^{b c e}\right) \text {. }
\end{aligned}
$$

Two and three products of these and the Jacobi identities (A.1)-(A.10) are given in [4]. The last Jacobi identity which was missing in [4] is included in (A.11). The four products between 
$d$ and $f$ symbols (A.12)-(A.17) are given in [42] with an appropriate normalization. In this paper, the identities (A.1)-(A.7), (A.10), (A.11) and (A.14) are used mainly and the other identities can be used for higher order representations of $S U(N)$.

\section{Appendix B The operator product expansions between the spin 1 current and primary spin 3 and 4 currents in subsection 2.2}

Let us continue to simplify the $\frac{1}{(z-w)^{2}}$ terms in the operator product expansion (2.23). First of all, by using the $d$ symbol (2.12) one can write down $k$-dependent term as follows:

$$
\begin{aligned}
d^{a b c d} J^{c} J^{d}(w) & =d^{a b c} Q^{c}(w)-\frac{\left(N^{2}-4\right)\left(N^{2}-3\right)}{N\left(N^{2}+1\right)} f^{a b c} \partial J^{c}(w)+2 d^{a c e} d^{b d e} J^{c} J^{d}(w) \\
& -\frac{8\left(N^{2}-4\right)}{N\left(N^{2}+1\right)} J^{a} J^{b}(w)-\frac{4\left(N^{2}-4\right)}{N\left(N^{2}+1\right)} \delta^{a b} J^{c} J^{c}(w) .
\end{aligned}
$$

Now let us consider the next term. The $d f f$ terms can be simplified by the identity (A.14).

$$
\begin{aligned}
d^{b c d e} f^{f d g} f^{a c f} J^{g} J^{e}(w) & =\frac{4\left(N^{2}-4\right)}{\left(N^{2}+1\right)} f^{a b c} \partial J^{c}(w)-\frac{4\left(2 N^{2}-3\right)}{N\left(N^{2}+1\right)} d^{a b c} Q^{c}(w) \\
& +\frac{4\left(N^{2}-4\right)\left(N^{2}+3\right)}{N^{2}\left(N^{2}+1\right)} J^{a} J^{b}(w)+\frac{4\left(N^{2}-4\right)\left(N^{2}-3\right)}{N^{2}\left(N^{2}+1\right)} \delta^{a b} J^{c} J^{c}(w) \\
& -\frac{2\left(N^{4}-3 N^{2}+6\right)}{N\left(N^{2}+1\right)} d^{a c e} d^{b d e} J^{c} J^{d}(w) .
\end{aligned}
$$

We also used the identity (A.11) for the $f f$ terms. For the next term, one writes down

$$
d^{b c d e} f^{f e g} f^{a c f} J^{d} J^{g}(w)=d^{b c d e} f^{f d g} f^{a c f} J^{g} J^{e}(w)+d^{b c d e} f^{f d g} f^{a c f} f^{e g h} \partial J^{h}(w),
$$

by interchanging an index $d$ and an index $e$ that are dummy indices. Moreover, the second term can be further simplified as before and this becomes $\frac{2\left(N^{2}-4\right)\left(N^{2}-9\right)}{\left(N^{2}+1\right)} f^{a b h} \partial J^{h}(w)$. Of course the first term of the equation $(\overline{B .3})$ is given by the relation (B.2). Similarly, one obtains the final term

$$
d^{b c d e} f^{f e g} f^{a d f} J^{c} J^{g}(w)=d^{b c d e} f^{f d g} f^{a c f} J^{g} J^{e}(w)+\frac{2\left(N^{2}-4\right)\left(N^{2}-9\right)}{\left(N^{2}+1\right)} f^{a b c} \partial J^{c}(w) .
$$

Therefore, by collecting the four terms characterized by (B.1), (B.2), (B.3) and (B.4), one arrives at the final simplified expression for the $\frac{1}{(z-w)^{2}}$ terms in the operator product expansion $J^{a}(z) T_{b}^{(3,1)}(w)$. See the operator product expansion (‥10) for the complete expression we will present below. 
Let us move on the $\frac{1}{(z-w)}$ terms. By multiplying $f^{a c f} J^{f}$ into the relation (B.1) to the left, one has the following expression

$$
\begin{aligned}
& f^{a c f} d^{b c d e} J^{f} J^{d} J^{e}(w)=f^{a c f} d^{b c d} J^{f} Q^{d}(w)-\frac{\left(N^{2}-4\right)\left(N^{2}-3\right)}{N\left(N^{2}+1\right)} f^{a c f} f^{b c d} J^{f} \partial J^{d}(w) \\
& -\frac{4\left(N^{2}-4\right)}{N\left(N^{2}+1\right)} f^{a b c} J^{c} J^{d} J^{d}(w)+2 f^{a c f} d^{b d g} d^{c e g} J^{f} J^{d} J^{e}(w)+\frac{8\left(N^{2}-4\right)}{\left(N^{2}+1\right)} J^{b} \partial J^{a}(w) \\
& -\frac{8\left(N^{2}-4\right)}{N\left(N^{2}+1\right)} f^{a c f} f^{f b g} \partial J^{g} J^{c}(w) .
\end{aligned}
$$

Here we also rewrite $f^{a c f} J^{f} J^{b} J^{c}$ term in terms of $J^{b} \partial J^{a}$ term and $f^{a c f} f^{f b g} \partial J^{g} J^{c}$ term. By moving the current $J^{f}$ to the left and interchanging between the index $d$ and the index $c$ that are dummy indices, one has

$$
d^{b c d e} f^{a d f} J^{c} J^{f} J^{e}(w)=f^{a c f} d^{b c d e} J^{f} J^{d} J^{e}(w)+d^{b c d e} f^{a d f} f^{c f g} \partial J^{g} J^{e}(w) .
$$

The first term of the equation (B.6) is equal to the expression (B.5). How does one determine the second term above? With 4 index $d$ symbol (2.12), one can use the identities (A.14) and (A.6) and it leads to

$$
\begin{aligned}
& d^{b c d e} f^{a d f} f^{c f g} \partial J^{g} J^{e}(w)=\frac{8\left(N^{2}-4\right)}{N^{2}\left(N^{2}+1\right)} \partial J^{a} J^{b}(w)+\frac{4\left(4-N^{2}\right)}{N^{2}} \delta^{a b} \partial J^{c} J^{c}(w) \\
& +\frac{2\left(N^{2}-4\right)}{N} d^{a c e} d^{b d e} \partial J^{c} J^{d}(w)+\frac{4}{N} d^{a b c} d^{c d e} \partial J^{d} J^{e}(w)+\frac{4\left(4-N^{2}\right)}{N^{2}} \partial J^{b} J^{a}(w) \\
& +\frac{4}{N} d^{a c e} d^{b d e} \partial J^{d} J^{c}(w)+\frac{4\left(N^{2}-4\right)}{N\left(N^{2}+1\right)} f^{a c e} f^{b d e} \partial J^{d} J^{c}(w)-\frac{4\left(N^{2}-4\right)}{N\left(N^{2}+1\right)} f^{a b e} f^{c d e} \partial J^{c} J^{d}(w) .
\end{aligned}
$$

One also has the following term by changing the index $d$ and the index $f$ that are dummy indices

$$
d^{b c d e} f^{a d f} J^{c} J^{f} J^{e}(w)=d^{b c f e} f^{a f d} J^{c} J^{d} J^{e}(w)=d^{b c d e} f^{a d f} J^{c} J^{f} J^{e}(w)
$$

where we changed the index $d$ and $f$ again and this is equal to the relation (B.5). Let us consider the final term by moving the current $J^{f}$ to the left.

$$
d^{b c d e} f^{a e f} J^{c} J^{d} J^{f}(w)=d^{b c d e} f^{a d f} J^{c} J^{f} J^{e}(w)+d^{b c d e} f^{a d f} f^{e f g} J^{c} \partial J^{g}(w),
$$

where the first term is exactly the same as the relation (B.5) via the relation (B.8) and one should simplify the second term. By moving the current $\partial J^{g}$ to the left, one gets that the second term is given by both the equation (B.7) and $-\frac{\left(N^{2}-4\right)\left(N^{2}-9\right)}{\left(N^{2}+1\right)} f^{a b c} \partial^{2} J^{c}(w)$. By combining the expressions given in $(\overline{\mathrm{B} .5}),(\overline{\mathrm{B} .6})$, and $(\overline{\mathrm{B} .9})$ altogether with correct multiplicities, one obtains the final expression which will be present in the operator product expansion (B.10). 
Then finally, one arrives at the following operator product expansion from the result in the previous subsection 2.2 and the results in this Appendix 13

$$
\begin{aligned}
& J^{a}(z) T_{b}^{(3,1)}(w)=-\frac{1}{(z-w)^{3}} \frac{2\left(N^{2}-4\right)\left(N^{2}-9\right)}{\left(N^{2}+1\right)} f^{a b c} J^{c} \\
& +\frac{1}{(z-w)^{2}}\left[\left(-3 k-\frac{12\left(2 N^{2}-3\right)}{N\left(N^{2}+1\right)}\right) d^{a b c} Q^{c}+\frac{\left(N^{2}-4\right)}{\left(N^{2}+1\right)}\left(\frac{3 k}{N}\left(N^{2}-3\right)+4\left(N^{2}-6\right)\right) f^{a b c} \partial J^{c}\right. \\
& +\left(-6 k-\frac{6}{N\left(N^{2}+1\right)}\left(N^{4}-3 N^{2}+6\right)\right) d^{a c e} d^{b d e} J^{c} J^{d} \\
& \left.+\frac{12\left(N^{2}-4\right)}{N\left(N^{2}+1\right)}\left(2 k+\frac{\left(N^{2}+3\right)}{N}\right) J^{a} J^{b}+\frac{12\left(N^{2}-4\right)}{N\left(N^{2}+1\right)}\left(k+\frac{\left(N^{2}-3\right)}{N}\right) \delta^{a b} J^{c} J^{c}\right] \\
& +\frac{1}{(z-w)}\left[-3 f^{a c e} d^{b d e} J^{c} Q^{d}-\frac{3\left(N^{2}-4\right)\left(N^{2}-3\right)}{N\left(N^{2}+1\right)} f^{a c e} f^{b d e} J^{c} \partial J^{d}+6 f^{a c f} d^{b d g} d^{c e g} J^{f} J^{d} J^{e}\right. \\
& +\frac{24\left(N^{2}-4\right)}{\left(N^{2}+1\right)} J^{b} \partial J^{a}+\frac{12\left(N^{2}-6\right)}{N\left(N^{2}+1\right)} f^{a c e} f^{b d e} \partial J^{d} J^{c}-\frac{12\left(N^{2}-4\right)}{N\left(N^{2}+1\right)} f^{a b c} J^{c} J^{d} J^{d} \\
& +\frac{24\left(N^{2}-4\right)}{N^{2}\left(N^{2}+1\right)} \partial J^{a} J^{b}-\frac{12\left(N^{2}-4\right)}{N^{2}} \delta^{a b} \partial J^{c} J^{c}+\frac{6\left(N^{2}-4\right)}{N} d^{a c e} d^{b d e} \partial J^{c} J^{d} \\
& +\frac{12}{N} d^{a b c} d^{c d e} \partial J^{d} J^{e}-\frac{12\left(N^{2}-4\right)}{N^{2}} \partial J^{b} J^{a}+\frac{12}{N} d^{a c e} d^{b d e} \partial J^{d} J^{c} \\
& \left.-\frac{12\left(N^{2}-4\right)}{N\left(N^{2}+1\right)} f^{a b c} f^{c d e} \partial J^{d} J^{e}-\frac{\left(N^{2}-4\right)\left(N^{2}-9\right)}{\left(N^{2}+1\right)} f^{a b c} \partial^{2} J^{c}\right]+\cdots
\end{aligned}
$$

Let us consider the $\frac{1}{(z-w)^{2}}$ term in (2.24). Let us first note

$$
\begin{aligned}
d^{a b c d} J^{b} J^{c} J^{d}(w) & =3 d^{a b c} J^{b} Q^{c}(w)-\frac{12\left(N^{2}-4\right)}{N\left(N^{2}+1\right)} J^{a} J^{b} J^{b}(w)+\frac{2\left(N^{2}-4\right)\left(N^{2}-3\right)}{N\left(N^{2}+1\right)} f^{a b c} \partial J^{b} J^{c}(w) \\
& -\frac{\left(N^{2}-4\right)\left(N^{2}-3\right)}{N\left(N^{2}+1\right)} f^{a b c} J^{b} \partial J^{c}(w) .
\end{aligned}
$$

This can be seen from the relation (B.1) by multiplying one more current $J^{b}$ where we use the identity (A.7) and rearrange the currents in order to simplify. The first term of $d$ symbol in (2.12) contributes to $-N d^{a b c} J^{b} Q^{c}(w)$ via the relation (A.6). The second and third terms of $d$ symbol in (2.12) can be used by the identity (A.14). The $J^{g} J^{d} J^{e}(w)$ term in the $\frac{1}{(z-w)^{2}}$ terms can be summarized by

$$
\begin{aligned}
f^{a b f} f^{f c g} d^{b c d e} J^{g} J^{d} J^{e}(w) & =-2 N d^{a b c} J^{b} Q^{c}(w)-\frac{12\left(N^{2}-4\right)}{N\left(N^{2}+1\right)} J^{a} J^{b} J^{b}(w)+\frac{4\left(N^{2}-4\right)}{N\left(N^{2}+1\right)} f^{a b c} J^{b} \partial J^{c}(w) \\
& -\frac{8\left(N^{2}-4\right)}{N\left(N^{2}+1\right)} f^{a b c} \partial J^{b} J^{c}(w) .
\end{aligned}
$$

\footnotetext{
${ }^{13}$ Sometimes we do not specify the argument of $w$ for the field on the right hand side of any operator product expansion as in (B.10) for simplicity.
} 
The $J^{c} J^{g} J^{e}(w)$ term consists of the relation ( $\left.\underline{\mathrm{B} .12}\right)$ and $-\frac{2\left(N^{2}-4\right)\left(N^{2}-9\right)}{\left(N^{2}+1\right)} f^{a b c} \partial J^{b} J^{c}(w)$. The $J^{c} J^{d} J^{g}(w)$ term can be written in terms of $J^{c} J^{g} J^{e}(w)$ term plus $\frac{2\left(N^{2}-4\right)\left(N^{2}-9\right)}{\left(N^{2}+1\right)} f^{a b c}\left(J^{b} \partial J^{c}(w)-\right.$ $\left.\partial J^{b} J^{c}(w)\right)$. Moreover the $J^{b} J^{g} J^{e}(w)$ term is the same as the $J^{c} J^{g} J^{e}(w)$ term, the $J^{b} J^{d} J^{g}(w)$ term becomes the $J^{c} J^{d} J^{g}(w)$ term and similarly, the $J^{b} J^{c} J^{g}(w)$ term becomes the $J^{c} J^{d} J^{g}(w)$ term. One obtains six $J^{g} J^{d} J^{e}(w)$ terms and $\frac{2\left(N^{2}-4\right)\left(N^{2}-9\right)}{\left(N^{2}+1\right)} f a b c\left(3 J^{b} \partial J^{c}(w)-5 \partial J^{b} J^{c}(w)\right)$ by combining all these terms. See the operator product expansion (B.13) for complete expression below.

Finally, one arrives at the following operator product expansion from the result in the previous subsection 2.2 and the results in this Appendix as follows:

$$
\begin{aligned}
J^{a}(z) T^{(4,0)}(w) & =\frac{1}{(z-w)^{4}} \frac{4 N\left(N^{2}-4\right)\left(N^{2}-9\right)}{\left(N^{2}+1\right)} J^{a}-\frac{1}{(z-w)^{3}} \frac{4 N\left(N^{2}-4\right)\left(N^{2}-9\right)}{\left(N^{2}+1\right)} \partial J^{a} \\
& +\frac{1}{(z-w)^{2}}\left[-12(N+k) d^{a b c} J^{b} Q^{c}+\frac{48\left(N^{2}-4\right)(N+k)}{N\left(N^{2}+1\right)} J^{a} J^{b} J^{b}\right. \\
& +\frac{2\left(N^{2}-4\right)}{N\left(N^{2}+1\right)}\left(3 N\left(N^{2}-5\right)+2 k\left(N^{2}-3\right)\right) f^{a b c} J^{b} \partial J^{c} \\
& \left.-\frac{2\left(N^{2}-4\right)}{N\left(N^{2}+1\right)}\left(N\left(5 N^{2}-21\right)+4 k\left(N^{2}-3\right)\right) f^{a b c} \partial J^{b} J^{c}\right]+\cdots
\end{aligned}
$$

There exist $\frac{1}{(z-w)}$ terms in the operator product expansion (B.13). They are

$$
d^{b c d e}\left[f^{a b f} J^{f} J^{c} J^{d} J^{e}(w)+f^{a c f} J^{b} J^{f} J^{d} J^{e}(w)+f^{a d f} J^{b} J^{c} J^{f} J^{e}(w)+f^{a e f} J^{b} J^{c} J^{d} J^{f}(w)\right] .
$$

Let us look at the first term of the relation (B.14). This can be written, by multiplying the current $J^{f}$ into the relation (B.11) with $f^{a b f}$, as follows:

$$
\begin{aligned}
& f^{a b f} d^{b c d e} J^{f} J^{c} J^{d} J^{e}(w)=-3 f^{a b c} d^{c d e} J^{b} J^{d} Q^{e}(w)+\frac{12\left(N^{2}-4\right)}{\left(N^{2}+1\right)} \partial J^{a} J^{b} J^{b}(w) \\
& +\frac{\left(N^{2}-4\right)\left(N^{2}-3\right)}{N\left(N^{2}+1\right)} f^{a b c} f^{c d e} J^{b} J^{d} \partial J^{e}(w)-\frac{2\left(N^{2}-4\right)\left(N^{2}-3\right)}{N\left(N^{2}+1\right)} f^{a b c} f^{c d e} J^{b} \partial J^{d} J^{e}(w) .
\end{aligned}
$$

In order to simplify the second term of (B.14), we move the current $J^{f}$ to the left and then the first term of (B.15) occurs and the extra piece has the following form

$$
\begin{aligned}
& f^{a b f} f^{c f g} d^{b c d e} \partial J^{g} J^{d} J^{e}(w)=\frac{2\left(N^{2}-4\right)\left(N^{2}-3\right)}{N\left(N^{2}+1\right)} d^{a b c} \partial J^{b} Q^{c}(w)+\frac{4\left(N^{2}-4\right)}{\left(N^{2}+1\right)} f^{a b c} \partial J^{b} \partial J^{c}(w) \\
& -\frac{24\left(N^{2}-4\right)}{N^{2}\left(N^{2}+1\right)} \partial J^{a} J^{b} J^{b}(w)-\frac{8\left(N^{2}-4\right)\left(N^{2}-3\right)}{N^{2}\left(N^{2}+1\right)} \partial J^{b} J^{b} J^{a}(w) \\
& +\frac{8\left(2 N^{2}-3\right)}{N\left(N^{2}+1\right)} d^{a b c} d^{c d e} \partial J^{d} J^{e} J^{b}(w) .
\end{aligned}
$$


In the relation (B.1), we have simplified expression and we multiply $\partial J^{g}$ with $f$ symbols. Using the identities (A.6), (A.5), (A.14) and (A.11) we obtain the right hand side of (B.16). For the third term of (B.14), we move the current $J^{f}$ to the left. Then we have the second term of (B.14), the extra piece (B.16) and $\frac{\left(N^{2}-4\right)\left(N^{2}-9\right)}{\left(N^{2}+1\right)} f^{a b c} \partial^{2} J^{b} J^{c}(w)$. The fourth term of (B.14) can be written as the third term of (B.14) and the extra piece (B.16) and $\frac{\left(N^{2}-4\right)\left(N^{2}-9\right)}{\left(N^{2}+1\right)} f^{a b c}\left(\partial^{2} J^{b} J^{c}(w)-J^{b} \partial J^{c}(w)\right)$.

It turns out that the final expression consists of four of (B.15), six of (B.16) and

$$
\frac{\left(N^{2}-4\right)\left(N^{2}-9\right)}{\left(N^{2}+1\right)} f^{a b c}\left[3 \partial^{2} J^{b} J^{c}(w)-J^{b} \partial^{2} J^{c}(w)\right] .
$$

So combining the relations $(\bar{B} .15),(\bar{B} .16)$ and $(\overline{B .17})$, it turns out to be

$$
\begin{aligned}
& -12 f^{a b c} d^{c d e} J^{b} J^{d} Q^{e}(w)+6\left(N^{2}-4\right) f^{a b c} J^{b} \partial^{2} J^{c}(w)+12 N d^{a b c} d^{c d e} \partial J^{d} J^{e} J^{b}(w) \\
& -2 N\left(N^{2}-4\right) \partial^{3} J^{a}(w) .
\end{aligned}
$$

For $N=4$, we have checked that the equation (B.18) vanishes.

The fundamental results of this Appendix will be used in next Appendices.

\section{Appendix C The operator product expansions between the spins 1,2 currents and various spin 4 cur- rents in subsection 2.2}

Using the identity $(A .15)$ of [4, one writes the following relation

$$
\left(J^{a} J^{a}\right)\left(J^{b} J^{b}\right)(z)=-2(k+N) \partial^{2} J^{a} J^{a}(z)+J^{a} J^{a} J^{b} J^{b}(z),
$$

and from the defining equation (2.2), one has

$$
\partial^{2} T(z)=-\frac{1}{(k+N)}\left[\partial J^{a} \partial J^{a}(z)+\partial^{2} J^{a} J^{a}(z)\right],
$$

and also the following relation holds

$$
Q^{a} Q^{a}(z)=d^{a b c} d^{a d e} J^{b} J^{c} J^{d} J^{e}(z)-\frac{2}{N}\left(N^{2}-4\right)(2 k+N) \partial^{2} J^{a} J^{a}(z) .
$$

We present some operator product expansions between the primary spin 1 current and other spin 4 currents as follows:

$$
J^{a}(z) Q^{b} Q^{b}(w)=\frac{1}{(z-w)^{4}} \frac{2}{N}\left(N^{2}-4\right)(N+2 k)(5 N+6 k) J^{a}
$$




$$
\begin{aligned}
& +\frac{1}{(z-w)^{3}} 2\left(N^{2}-4\right)(N+2 k) \partial J^{a} \\
& +\frac{1}{(z-w)^{2}}\left[-2(N+k) d^{a b c}\left(J^{b} Q^{c}+Q^{b} J^{c}\right)\right. \\
& \left.+\frac{\left(N^{2}-4\right)(N+2 k)}{N} f^{a b c}\left(\partial J^{b} J^{c}-J^{b} \partial J^{c}\right)\right]+\cdots, \\
J^{a}(z) T^{2}(w) & =\frac{1}{(z-w)^{4}} 3 J^{a}+\frac{1}{(z-w)^{2}}\left[J^{a} T+T J^{a}\right]+\cdots, \\
J^{a}(z) \partial^{2} T(w) & =\frac{1}{(z-w)^{4}} 6 J^{a}+\frac{1}{(z-w)^{3}} 4 \partial J^{a}+\frac{1}{(z-w)^{2}} \partial^{2} J^{a}+\cdots, \\
J^{a}(z) \partial^{2} J^{b} J^{b}(w) & =-\frac{1}{(z-w)^{4}} 2(2 N+3 k) J^{a}-\frac{1}{(z-w)^{3}} 2 N \partial J^{a} \\
& +\frac{1}{(z-w)^{2}}\left[-2 f^{a b c} \partial J^{b} J^{c}-k \partial^{2} J^{a}\right]+\cdots
\end{aligned}
$$

Then one obtains the operator product expansions between $J^{a}(z)$ and the currents $J^{b} J^{b} J^{c} J^{c}(w)$, $\partial J^{b} \partial J^{b}(w)$ and $d^{b c f} d^{d e f} J^{b} J^{c} J^{d} J^{e}(w)$ through the relations (C.4), (C.5), (C.6), and (C.7). We also consider some operator product expansions between the stress energy tensor and other spin 4 currents as follows:

$$
\begin{aligned}
T(z) T^{2}(w) & =\frac{1}{(z-w)^{6}} \frac{3 k\left(N^{2}-1\right)}{N+k}+\frac{1}{(z-w)^{4}}\left[8+\frac{k}{N+k}\left(N^{2}-1\right)\right] T \\
& +\frac{1}{(z-w)^{3}} 3 \partial T+\frac{1}{(z-w)^{2}} 4 T^{2}+\frac{1}{(z-w)} \partial T^{2}+\cdots, \\
T(z) \partial^{2} T(w) & =\frac{1}{(z-w)^{6}} \frac{10 k}{N+k}\left(N^{2}-1\right)+\frac{1}{(z-w)^{4}} 12 T+\frac{1}{(z-w)^{3}} 10 \partial T \\
& +\frac{1}{(z-w)^{2}} 4 \partial^{2} T+\frac{1}{(z-w)} \partial^{3} T+\cdots, \\
T(z) \partial^{2} J^{b} J^{b}(w) & =-\frac{1}{(z-w)^{6}} 6 k\left(N^{2}-1\right)+\frac{1}{(z-w)^{4}} 6 J^{a} J^{a}+\frac{1}{(z-w)^{3}} 6 \partial J^{a} J^{a} \\
& +\frac{1}{(z-w)^{2}} 4 \partial^{2} J^{a} J^{a}+\frac{1}{(z-w)} \partial\left(\partial^{2} J^{a} J^{a}\right)+\cdots
\end{aligned}
$$

The operator product expansion $T(z) Q^{a} Q^{a}(w)$ is given in (2.26). The operator product expansions between $T(z)$ and the currents $J^{b} J^{b} J^{c} J^{c}(w), \partial J^{b} \partial J^{b}(w)$ and $d^{b c f} d^{d e f} J^{b} J^{c} J^{d} J^{e}(w)$ can be obtained similarly from the above relations (C.8), (C.9) and (C.10). One should have the operator product expansions between the coset stress energy tensor (3.6) with (3.7) and other currents in next Appendices. Then it is better to write down the relations (C.8), (C.9) and (C.10) in terms of the coset central charge (3.9). For example, the operator product expansion $\widetilde{T}(z) \widetilde{T}^{2}(w)$ can be read off from the equation (C.8) by taking the central charge $c$ (2.4) as the coset central charge $\widetilde{c}($ (3.9) $)$ and the stress energy tensor $T(w)$ as the coset stress 
energy tensor $\widetilde{T}(w)$ on the right hand side of (C.8). It is not right to replace $k$ with $k_{1}+k_{2}$ and $T(w)$ as $\widetilde{T}(w)$. That is, the $\frac{1}{(z-w)^{4}}$ term of the operator product expansion $\widetilde{T}(z) \widetilde{T}^{2}(w)$ is $(8+\widetilde{c}) \widetilde{T}(w)$ that is not equal to $\left[8+\frac{k_{1}+k_{2}}{N+k_{1}+k_{2}}\left(N^{2}-1\right)\right] \widetilde{T}(w)$.

\section{Appendix D The operator product expansions between the diagonal spin 1 current and various spin 4 currents in subsection 3.2}

We summarize the operator product expansions between the diagonal primary spin 1 current (3.4) and various spin 4 currents as follows:

$$
\begin{aligned}
& J^{\prime a}(z) d^{b c d e} J^{b} J^{c} J^{d} J^{e}(w)=\frac{1}{(z-w)^{4}} \frac{4 N\left(N^{2}-4\right)\left(N^{2}-9\right)}{\left(N^{2}+1\right)} J^{a} \\
& -\frac{1}{(z-w)^{3}} \frac{4 N\left(N^{2}-4\right)\left(N^{2}-9\right)}{\left(N^{2}+1\right)} \partial J^{a}+\frac{1}{(z-w)^{2}}\left[-12\left(N+k_{1}\right) d^{a b c} J^{b} Q^{c}\right. \\
& +\frac{48\left(N^{2}-4\right)\left(N+k_{1}\right)}{N\left(N^{2}+1\right)} J^{a} J^{b} J^{b}+\frac{2\left(N^{2}-4\right)}{N\left(N^{2}+1\right)}\left(3 N\left(N^{2}-5\right)+2 k_{1}\left(N^{2}-3\right)\right) f^{a b c} J^{b} \partial J^{c} \\
& \left.-\frac{2\left(N^{2}-4\right)}{N\left(N^{2}+1\right)}\left(N\left(5 N^{2}-21\right)+4 k_{1}\left(N^{2}-3\right)\right) f^{a b c} \partial J^{b} J^{c}\right]+\cdots \\
& J^{\prime a}(z) d^{b c d e} J^{b} J^{c} J^{d} K^{e}(w)=\frac{1}{(z-w)^{3}} \frac{2\left(N^{2}-4\right)\left(N^{2}-9\right)}{\left(N^{2}+1\right)} f^{a b c} J^{b} K^{c} \\
& +\frac{1}{(z-w)^{2}}\left[\left(-3 k_{1}-\frac{12\left(2 N^{2}-3\right)}{N\left(N^{2}+1\right)}\right) d^{a b c} Q^{b} K^{c}-\frac{\left(N^{2}-4\right)}{\left(N^{2}+1\right)}\left(\frac{3 k_{1}\left(N^{2}-3\right)}{N}+4\left(N^{2}-6\right)\right) f^{a b c} \partial J^{b} K^{c}\right. \\
& -6\left(k_{1}+\frac{N^{4}-3 N^{2}+6}{N\left(N^{2}+1\right)}\right) d^{a c e} d^{b d e} J^{c} J^{d} K^{b}+\frac{12\left(N^{2}-4\right)}{N\left(N^{2}+1\right)}\left(2 k_{1}+\frac{N^{2}+3}{N}\right) J^{a} J^{b} K^{b} \\
& +\frac{12\left(N^{2}-4\right)}{N\left(N^{2}+1\right)}\left(k_{1}+\frac{N^{2}-3}{N}\right) J^{b} J^{b} K^{a}-3 k_{2} d^{a b c} J^{b} Q^{c} \\
& \left.+\frac{12 k_{2}\left(N^{2}-4\right)}{N\left(N^{2}+1\right)} J^{a} J^{b} J^{b}-\frac{k_{2}\left(N^{2}-4\right)\left(N^{2}-3\right)}{N\left(N^{2}+1\right)} f^{a b c}\left(2 \partial J^{b} J^{c}-J^{b} \partial J^{c}\right)\right]+\cdots \\
& J^{\prime a}(z) d^{b c d e} J^{b} J^{c} K^{d} K^{e}(w)=\frac{1}{(z-w)^{2}}\left[-\left(4 k_{1}+\frac{8\left(2 N^{2}-3\right)}{N\left(N^{2}+1\right)}\right) d^{a c e} d^{b d e} J^{b} K^{c} K^{d}\right. \\
& -2\left(k_{1}+\frac{\left(N^{2}-4\right)\left(N^{2}-3\right)}{N\left(N^{2}+1\right)}\right) d^{a b c} J^{b} R^{c}+\frac{2\left(N^{2}-4\right)}{N^{2}+1}\left(\frac{k_{1}\left(N^{2}-3\right)}{N}+2\right) f^{a b c} J^{b} \partial K^{c} \\
& +\frac{8\left(N^{2}-4\right)}{N\left(N^{2}+1\right)}\left(2 k_{1}+\frac{N^{2}-3}{N}\right) J^{b} K^{a} K^{b}+\frac{8\left(N^{2}-4\right)}{N\left(N^{2}+1\right)}\left(k_{1}+\frac{3}{N}\right) J^{a} K^{b} K^{b} \\
& -2\left(k_{2}+\frac{\left(N^{2}-4\right)\left(N^{2}-3\right)}{N\left(N^{2}+1\right)}\right) d^{a b c} Q^{b} K^{c}-\frac{2\left(N^{2}-4\right)}{N^{2}+1}\left(\frac{k_{2}\left(N^{2}-3\right)}{N}+2\right) f^{a b c} \partial J^{b} K^{c} \\
& N .2) \\
& \\
& +1
\end{aligned}
$$




$$
\begin{aligned}
& +\frac{8\left(N^{2}-4\right)}{N\left(N^{2}+1\right)}\left(2 k_{2}+\frac{N^{2}-3}{N}\right) J^{a} J^{b} K^{b}+\frac{8\left(N^{2}-4\right)}{N\left(N^{2}+1\right)}\left(k_{2}+\frac{3}{N}\right) J^{b} J^{b} K^{a} \\
& \left.-4\left(k_{2}+\frac{2\left(2 N^{2}-3\right)}{N\left(N^{2}+1\right)}\right) d^{a c e} d^{b d e} J^{c} J^{d} K^{b}\right]+\cdots, \\
& J^{\prime a}(z) d^{b c d e} J^{b} K^{c} K^{d} K^{e}(w)=\left.J^{\prime a}(z) d^{b c d e} J^{b} J^{c} J^{d} K^{e}(w)\right|_{k_{1} \leftrightarrow k_{2}, J^{a} \leftrightarrow K^{a}}, \\
& J^{\prime a}(z) d^{b c d e} K^{b} K^{c} K^{d} K^{e}(w)=\left.J^{\prime a}(z) d^{b c d e} J^{b} J^{c} J^{d} J^{e}(w)\right|_{k_{1} \leftrightarrow k_{2}, J^{a} \leftrightarrow K^{a}}, \\
& J^{\prime a}(z) d^{b c f} d^{d e f} J^{b} J^{c} J^{d} J^{e}(w)=\frac{1}{(z-w)^{4}} 2\left(N^{2}-4\right)\left(N+2 k_{1}\right) J^{a} \\
& -\frac{1}{(z-w)^{3}} 2\left(N^{2}-4\right)\left(N+2 k_{1}\right) \partial J^{a}+\frac{1}{(z-w)^{2}}\left[-4\left(N+k_{1}\right) d^{a b c} J^{b} Q^{c}\right. \\
& \left.-\frac{\left(N^{2}-4\right)\left(N+2 k_{1}\right)}{N} f^{a b c}\left(\partial J^{b} J^{c}-J^{b} \partial J^{c}\right)\right]+\cdots, \\
& J^{\prime a}(z) d^{b c f} d^{d e f} J^{b} J^{c} J^{d} K^{e}(w)=\frac{1}{(z-w)^{4}} 2 k_{1}\left(N^{2}-4\right) K^{a}+\frac{1}{(z-w)^{3}}\left(1-\frac{2 k_{1}}{N}\right)\left(N^{2}-4\right) f^{a b c} J^{b} K^{c} \\
& +\frac{1}{(z-w)^{2}}\left[\left(-\left(N+k_{1}\right)+\frac{2}{N}\left(N^{2}-4\right)\right) d^{a b c} Q^{b} K^{c}\right. \\
& +\left(-\left(N+2 k_{1}\right)-\frac{2}{N}\left(N^{2}-4\right)\right) d^{a c e} d^{b d e} J^{c} J^{d} K^{b}-\left(N^{2}-4\right) f^{a b c} \partial J^{b} K^{c}-k_{2} d^{a b c} J^{b} Q^{c} \\
& \left.-\frac{k_{2}\left(N^{2}-4\right)}{N} f^{a b c}\left(\partial J^{b} J^{c}-J^{b} \partial J^{c}\right)-\frac{8\left(N^{2}-4\right)}{N^{2}}\left(J^{a} J^{b} K^{b}-J^{b} J^{b} K^{a}\right)\right]+\cdots, \\
& J^{\prime a}(z) d^{b c f} d^{d e f} J^{b} J^{c} K^{d} K^{e}(w)=-\frac{1}{(z-w)^{2}}\left[\left(N+2 k_{1}\right) d^{a b c} J^{b} R^{c}+\left(N+2 k_{2}\right) d^{a b c} Q^{b} K^{c}\right] \\
& +\cdots, \\
& J^{\prime a}(z) d^{b c f} d^{d e f} J^{b} K^{c} K^{d} K^{e}(w)=-\frac{1}{(z-w)^{3}} \frac{1}{N}\left(N^{2}-4\right)\left(N+2 k_{2}\right) f^{a b c} J^{b} K^{c}+\frac{1}{(z-w)^{2}}[ \\
& -k_{1} d^{a b c} K^{b} R^{c}-\left(N+k_{2}\right) d^{a b c} J^{b} R^{c}-\left(N+2 k_{2}\right) d^{a c e} d^{b d e} J^{b} K^{c} K^{d} \\
& \left.+\frac{1}{N}\left(N^{2}-4\right)\left(N+2 k_{2}\right) f^{a b c} J^{b} \partial K^{c}\right]+\cdots, \\
& J^{\prime a}(z) d^{b c f} d^{d e f} K^{b} K^{c} K^{d} K^{e}(w)=\left.J^{\prime a}(z) d^{b c f} d^{d e f} J^{b} J^{c} J^{d} J^{e}(w)\right|_{k_{1} \leftrightarrow k_{2}, J^{a} \leftrightarrow K^{a}}, \\
& J^{\prime a}(z) J^{b} J^{b} J^{c} J^{c}(w)=\frac{1}{(z-w)^{4}} 4 N\left(N+k_{1}\right) J^{a}-\frac{1}{(z-w)^{3}} 4 N\left(N+k_{1}\right) \partial J^{a} \\
& +\frac{1}{(z-w)^{2}}\left[-4\left(N+k_{1}\right) J^{a} J^{b} J^{b}-2\left(N+k_{1}\right) f^{a b c} \partial J^{b} J^{c}+2\left(N+k_{1}\right) f^{a b c} J^{b} \partial J^{c}\right]+\cdots, \\
& J^{\prime a}(z) J^{b} J^{b} J^{c} K^{c}(w)=\frac{1}{(z-w)^{4}} 2 N k_{1} K^{a}+\frac{1}{(z-w)^{3}} 2\left(N-k_{1}\right) f^{a b c} J^{b} K^{c} \\
& +\frac{1}{(z-w)^{2}}\left[-k_{2} J^{a} J^{b} J^{b}-k_{2} f^{a b c} \partial J^{b} J^{c}+k_{2} f^{a b c} J^{b} \partial J^{c}+\left(-k_{1}+\frac{8}{N}\right) J^{b} J^{b} K^{a}\right. \\
& \left.-\left(2\left(N+k_{1}\right)+\frac{8}{N}\right) J^{a} J^{b} K^{b}-2 N f^{a b c} \partial J^{b} K^{c}-2 d^{a c e} d^{b d e} J^{c} J^{d} K^{b}+2 d^{a b c} Q^{b} K^{c}\right]+\cdots,
\end{aligned}
$$




$$
\begin{aligned}
& J^{\prime a}(z) J^{b} J^{b} K^{c} K^{c}(w)=-\frac{1}{(z-w)^{2}} 2\left[\left(N+k_{1}\right) J^{a} K^{b} K^{b}+\left(N+k_{2}\right) J^{b} J^{b} K^{a}\right]+\cdots, \\
& J^{\prime a}(z) J^{b} K^{b} K^{c} K^{c}(w)=-\frac{1}{(z-w)^{3}} 2\left(N+k_{2}\right) f^{a b c} J^{b} K^{c}+\frac{1}{(z-w)^{2}}\left[-k_{1} K^{a} K^{b} K^{b}\right. \\
& \left.-k_{2} J^{a} K^{b} K^{b}-2\left(N+k_{2}\right) J^{b} K^{a} K^{b}+2\left(N+k_{2}\right) f^{a b c} J^{b} \partial K^{c}\right]+\cdots, \\
& J^{\prime a}(z) K^{b} K^{b} K^{c} K^{c}(w)=\left.J^{\prime a}(z) K^{b} K^{b} K^{c} K^{c}(w)\right|_{k_{1} \leftrightarrow k_{2}, J^{a} \leftrightarrow K^{a}}, \\
& J^{\prime a}(z) \partial^{2} J^{b} J^{b}(w)=-\frac{1}{(z-w)^{4}} 2\left(2 N+3 k_{1}\right) J^{a}-\frac{1}{(z-w)^{3}} 2 N \partial J^{a} \\
& +\frac{1}{(z-w)^{2}}\left[-\frac{k_{1}}{N} f^{a b c} J^{b} \partial J^{c}-\left(2+\frac{k_{1}}{N}\right) f^{a b c} \partial J^{b} J^{c}\right]+\cdots, \\
& J^{\prime a}(z) \partial^{2} J^{b} K^{b}(w)=-\frac{1}{(z-w)^{4}} 6 k_{1} K^{a}-\frac{1}{(z-w)^{3}} f^{a b c} J^{b} K^{c} \\
& +\frac{1}{(z-w)^{2}}\left[-\frac{k_{2}}{N} f^{a b c}\left(\partial J^{b} J^{c}+J^{b} \partial J^{c}\right)-2 f^{a b c} \partial J^{b} K^{c}\right]+\cdots, \\
& J^{\prime a}(z) \partial^{2} K^{b} K^{b}(w)=\left.J^{\prime a}(z) \partial^{2} J^{b} J^{b}(w)\right|_{k_{1} \leftrightarrow k_{2}, J^{a} \leftrightarrow K^{a}}, \\
& J^{\prime a}(z) \partial J^{b} \partial J^{b}(w)=-\frac{1}{(z-w)^{4}} 2 N J^{a}-\frac{1}{(z-w)^{3}} 2\left(N+2 k_{1}\right) \partial J^{a} \\
& +\frac{1}{(z-w)^{2}} f^{a b c}\left(\partial J^{b} J^{c}-J^{b} \partial J^{c}\right)+\cdots, \\
& J^{\prime a}(z) \partial J^{b} \partial K^{b}(w)=-\frac{1}{(z-w)^{3}} 2\left[k_{1} \partial K^{a}+k_{2} \partial J^{a}\right]+\frac{1}{(z-w)^{2}} f^{a b c}\left(\partial J^{b} K^{c}-J^{b} \partial K^{c}\right) \\
& +\cdots, \\
& J^{\prime a}(z) \partial K^{b} \partial K^{c}(w)=\left.J^{\prime a}(z) \partial J^{b} \partial J^{c}(w)\right|_{k_{1} \leftrightarrow k_{2}, J^{a} \leftrightarrow K^{a}}, \\
& J^{\prime a}(z) J^{b} \partial^{2} K^{b}(w)=\left.J^{\prime a}(z) \partial^{2} J^{b} K^{b}(w)\right|_{k_{1} \leftrightarrow k_{2}, J^{a} \leftrightarrow K^{a}} .
\end{aligned}
$$

In the operator product expansion (D.1), since the current $K^{a}(z)$ commutes with $J^{b}(z)$ 's, one gets this from the operator product expansion (B.13) exactly 14 . In the operator product expansion ( $(\overline{\mathrm{D} .2})$, one uses the operator product expansion (B.10), the defining equation (3.3) and the relation ( $(\overline{B .11})$. In the operator product expansion (D.3), one can use the operator product expansion (2.22) and its version for the $K^{a}$ current, that is, the operator product expansion between $K^{a}(z)$ and $d^{b c d e} K^{d} K^{e}(w)$. Then one should simplify further. The following

\footnotetext{
${ }^{14}$ Sometimes it is better to describe the operator product expansions for arbitrary $k_{1}$ and $k_{2}$ instead of putting $k_{1}=1, k_{2}=k$ due to the fact that by using the symmetry between these levels and the symmetry of the two currents $J^{a}(z), K^{a}(z)$, some of the operator product expansions can be determined by known operator product expansions. For example, in (D.4), we do not have to compute the operator product expansion newly. Once the operator product expansion $J^{\prime a}(z) d^{b c d e} J^{b} J^{c} J^{d} K^{e}(w)$ is known, then the operator product expansion $J^{\prime a}(z) d^{b c d e} J^{b} K^{c} K^{d} K^{e}(w)$ is automatically determined by taking $J^{a} \leftrightarrow K^{a}$ and $k_{1} \leftrightarrow k_{2}$ on the above known operator product expansion. However, once $k_{1}=1$ and $k_{2}=k$ are fixed from the beginning, one should compute the operator product expansion $J^{\prime a}(z) d^{b c d e} J^{b} K^{c} K^{d} K^{e}(w)$ independently because one cannot find $k_{1}$ dependence.
} 
triple product $d J K K$ can be obtained from the relation (B.1) by replacing the current $J^{a}(w)$ 's with $K^{a}(w)$ 's and then we multiply $J^{b}$ current to the left with $d$ symbol as follows:

$$
\begin{aligned}
d^{a b c d} J^{b} K^{c} K^{d}(w) & =2 d^{a c e} d^{b d e} J^{b} K^{c} K^{d}(w)+d^{a b c} J^{b} R^{c}(w)-\frac{\left(N^{2}-4\right)\left(N^{2}-3\right)}{N\left(N^{2}+1\right)} f^{a b c} J^{b} \partial K^{c}(w) \\
& -\frac{4\left(N^{2}-4\right)}{N\left(N^{2}+1\right)}\left[2 J^{b} K^{a} K^{b}(w)+J^{a} K^{b} K^{b}(w)\right],
\end{aligned}
$$

where we introduce the spin 2 field for the $K^{a}(w)$ current corresponding to (2.7)

$$
R^{a}(w) \equiv d^{a b c} K^{b} K^{c}(w)
$$

One also has the following relation

$$
\begin{aligned}
f^{a d f} f^{f e g} d^{b c d e} J^{g} K^{b} K^{c}(w) & =-\frac{2\left(N^{2}-4\right)\left(N^{2}-3\right)}{N\left(N^{2}+1\right)} d^{a b c} J^{b} R^{c}(w)+\frac{4\left(N^{2}-4\right)}{\left(N^{2}+1\right)} f^{a b c} J^{b} \partial K^{c}(w) \\
& +\frac{24\left(N^{2}-4\right)}{N^{2}\left(N^{2}+1\right)} J^{a} K^{b} K^{b}(w)+\frac{8\left(N^{2}-4\right)\left(N^{2}-3\right)}{N^{2}\left(N^{2}+1\right)} J^{b} K^{a} K^{b}(w) \\
& -\frac{8\left(2 N^{2}-3\right)}{N\left(N^{2}+1\right)} d^{a c e} d^{b d e} J^{b} K^{c} K^{d}(w)
\end{aligned}
$$

where the relation $(\overline{\mathrm{B} .1})$ is used, the $J^{a}$ 's are exchanged with the current $K^{a}$ 's as before, the current $J^{a}$ is multiplied and the spin 2 field (D.24) is used. Then the identities (A.6), (A.14), (A.7) and (A.11) are used. Similarly, one can write down

$$
\begin{aligned}
d^{a b c d} J^{b} J^{c} K^{d}(w) & =2 d^{a d e} d^{b c e} J^{b} J^{c} K^{d}(w)+d^{a b c} K^{b} Q^{c}(w)+\frac{\left(N^{2}-4\right)\left(N^{2}-3\right)}{N\left(N^{2}+1\right)} f^{a b c} \partial J^{b} K^{c}(w) \\
& -\frac{4\left(N^{2}-4\right)}{N\left(N^{2}+1\right)}\left[2 J^{a} J^{b} K^{b}(w)+J^{b} J^{b} K^{a}(w)\right],
\end{aligned}
$$

which can be seen from the identity (B.1) and by multiplying two $f$ symbols into (D.26) one has the relation

$$
\begin{aligned}
f^{a d f} f^{f e g} d^{b c d e} J^{b} J^{c} K^{g}(w) & =-\frac{2\left(N^{2}-4\right)\left(N^{2}-3\right)}{N\left(N^{2}+1\right)} d^{a b c} Q^{b} K^{c}(w)-\frac{4\left(N^{2}-4\right)}{\left(N^{2}+1\right)} f^{a b c} \partial J^{b} K^{c}(w) \\
& +\frac{24\left(N^{2}-4\right)}{N^{2}\left(N^{2}+1\right)} J^{b} J^{b} K^{a}(w)+\frac{8\left(N^{2}-4\right)\left(N^{2}-3\right)}{N^{2}\left(N^{2}+1\right)} J^{a} J^{b} K^{b}(w) \\
& -\frac{8\left(2 N^{2}-3\right)}{N\left(N^{2}+1\right)} d^{a c e} d^{b d e} J^{c} J^{d} K^{b}(w) .
\end{aligned}
$$

In the operator product expansion (D.4), one has the following relation

$$
\begin{aligned}
d^{a b c d} K^{b} K^{c} K^{d}(w) & =3 d^{a b c} K^{b} R^{c}(w)-\frac{12\left(N^{2}-4\right)}{N\left(N^{2}+1\right)} K^{a} K^{b} K^{b}(w)+\frac{2\left(N^{2}-4\right)\left(N^{2}-3\right)}{N\left(N^{2}+1\right)} f^{a b c} \partial K^{b} K^{c}(w) \\
& -\frac{\left(N^{2}-4\right)\left(N^{2}-3\right)}{N\left(N^{2}+1\right)} f^{a b c} K^{b} \partial K^{c}(w)
\end{aligned}
$$


This can be seen from the relation (B.11) by replacing the currents $J^{a}$ 's with the currents $K^{a}$ 's. In the operator product expansion (D.6), one can use the relations ([C.3), (C.4) and (C.7). In the operator product expansion (D.7), one also need to have

$$
d^{a d e} d^{b c e} J^{b} J^{c} J^{d}(w)=d^{a b c} J^{b} Q^{c}(w)+\frac{N^{2}-4}{N} f^{a b c}\left[\partial J^{b} J^{c}(w)-J^{b} \partial J^{c}(w)\right] .
$$

One can check this by moving the current $J^{d}$ to the left together with the identity (A.7). One also has the following relation

$$
\begin{aligned}
f^{c e g} f^{a g h} J^{h} J^{c} K^{e}(w) & =d^{a b c} Q^{b} K^{c}(w)-\frac{4}{N}\left[J^{a} J^{b} K^{b}(w)-J^{b} J^{b} K^{a}(w)\right] \\
& -d^{a c e} d^{b d e} J^{c} J^{d} K^{b}(w)-N f^{a b c} \partial J^{b} K^{c}(w),
\end{aligned}
$$

where the identities (A.11) and (A.7) are used. In the operator product expansion (D.8), one uses the defining equation (2.9). In the operator product expansion (D.9), we also used the following relation

$$
d^{b c f} d^{a f g} J^{b} K^{c} K^{g}(w)=d^{a c e} d^{b d e} J^{b} K^{c} K^{d}(w)-\frac{N^{2}-4}{N} f^{a b c} J^{b} \partial K^{c}(w) .
$$

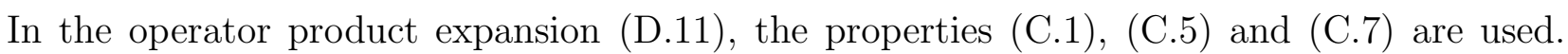
From the relation,

$$
J^{a} J^{a} J^{b}(w)=-2\left(N+k_{1}\right) J^{b} T_{(1)}(w)+2 f^{b c d} \partial J^{c} J^{d}(w)-N \partial^{2} J^{b}(w),
$$

one can compute the operator product expansion (D.12) where the following operator product expansions are used

$$
\begin{aligned}
J^{a}(z) J^{b} T_{(1)}(w) & =\frac{1}{(z-w)^{3}} f^{a b c} J^{c}+\frac{1}{(z-w)^{2}}\left[-k_{1} \delta^{a b} T_{(1)}+J^{b} J^{a}\right], \\
& +\frac{1}{(z-w)} f^{a b c} J^{c} T_{(1)}+\cdots, \\
J^{a}(z) f^{b c d} \partial J^{c} J^{d}(w) & =-\frac{1}{(z-w)^{4}} 2 N k_{1} \delta^{a b}+\frac{1}{(z-w)^{3}}\left(N+2 k_{1}\right) f^{a b c} J^{c} \\
& +\frac{1}{(z-w)^{2}}\left[f^{a c e} f^{b c d} J^{e} J^{d}-k_{1} f^{a b c} \partial J^{c}\right] \\
& +\frac{1}{(z-w)}\left[f^{a c e} f^{b c d} \partial J^{e} J^{d}-f^{a c e} f^{b c d} \partial J^{d} J^{e}\right]+\cdots
\end{aligned}
$$

In the operator product expansion (D.13), the operator product expansion between $J^{a}(z)$ and $T_{(1)}(w)$ is used. In the operator product expansion (D.14) , the first equation of (D.33) (with the current $K^{a}$ ) can be used: that is, the operator product expansion $K^{a}(z) K^{b} T_{(2)}(w)$. 
In the operator product expansion (D.16), the operator product expansion (C.7) is used. In the operator product expansion (D.17), the defining equation (2.1) is used. Moreover, in the operator product expansion (D.19), the relations (C.2), (‥6) and (C.7) are used. In the operator product expansion (D.20), the relation (2.1) is used.

Therefore, the independent field contents from the equations (B.11), (D.23), (D.25), $(\overline{\mathrm{D} .26}),(\mathrm{D} .27),(\overline{\mathrm{D} .28}),(\mathrm{D} .29),(\overline{\mathrm{D} .30})$ and $(\mathrm{D} .31)$ can be summarized by eighteen fields in the $\frac{1}{(z-w)^{2}}$ terms in the operator product expansions between the diagonal spin 1 current and spin 4 currents. See also the expression $(\underline{\mathrm{F} .2})$ we will explain in next Appendix.

\section{Appendix E The operator product expansions between the stress tensor and various spin 4 currents in subsection 3.2}

Let us introduce the following simplified notation as in (3.18)

$$
T_{(1)}(z)+T_{(2)}(z) \equiv \hat{T}(z) .
$$

Any spin 4 field $\Phi(z)$ satisfies $\hat{T}(z) \Phi(w)=\cdots+\frac{1}{(z-w)^{2}} 4 \Phi(w)+\frac{1}{(z-w)} \partial \Phi(w)+\cdots$. For simplicity, we present the only higher order singular terms $\frac{1}{(z-w)^{n}}$ with $n>2$ and we assume $\frac{1}{(z-w)^{2}}$ and $\frac{1}{(z-w)}$ terms as above:

$$
\begin{aligned}
& \hat{T}(z) d^{b c d e} J^{b} J^{c} J^{d} J^{e}(w)=+\cdots \\
& \hat{T}(z) d^{b c d e} J^{b} J^{c} J^{d} K^{e}(w)=+\cdots \\
& \hat{T}(z) d^{b c d e} J^{b} J^{c} K^{d} K^{e}(w)=+\cdots \\
& \hat{T}(z) d^{b c d e} J^{b} K^{c} K^{d} K^{e}(w)=+\cdots \\
& \hat{T}(z) d^{b c d e} K^{b} K^{c} K^{d} K^{e}(w)=+\cdots \\
& \hat{T}(z) d^{b c f} d^{d e f} J^{b} J^{c} J^{d} J^{e}(w)=-\frac{1}{(z-w)^{4}} \frac{4}{N}\left(N^{2}-4\right)\left(N+2 k_{1}\right) J^{a} J^{a}+\cdots \\
& \hat{T}(z) d^{b c f} d^{d e f} J^{b} J^{c} J^{d} K^{e}(w)=-\frac{1}{(z-w)^{4}} \frac{4 k_{1}}{N}\left(N^{2}-4\right) J^{a} K^{a} \\
& -\frac{1}{(z-w)^{3}} 2\left(N^{2}-4\right) \partial J^{a} K^{a}+\cdots, \\
& \hat{T}(z) d^{b c f} d^{d e f} J^{b} J^{c} K^{d} K^{e}(w)=+\cdots, \\
& \hat{T}(z) d^{b c f} d^{d e f} J^{b} K^{c} K^{d} K^{e}(w)=-\frac{1}{(z-w)^{4}} \frac{2}{N}\left(N^{2}-4\right)\left(N+2 k_{2}\right) J^{a} K^{a}+\cdots, \\
& \hat{T}(z) d^{b c f} d^{d e f} K^{b} K^{c} K^{d} K^{e}(w)=\left.\hat{T}(z) d^{b c f} d^{d e f} J^{b} J^{c} J^{d} J^{e}(w)\right|_{k_{1} \leftrightarrow k_{2}, J^{a} \leftrightarrow K^{a},}
\end{aligned}
$$




$$
\begin{aligned}
& \hat{T}(z) J^{b} J^{b} J^{c} J^{c}(w)=-\frac{1}{(z-w)^{4}} 2\left[2\left(N+k_{1}\right)+k_{1}\left(N^{2}-1\right)\right] J^{a} J^{a}+\cdots, \\
& \hat{T}(z) J^{b} J^{b} J^{c} K^{c}(w)=-\frac{1}{(z-w)^{4}} k_{1}\left(N^{2}+1\right) J^{a} K^{a}-\frac{1}{(z-w)^{3}} 2 N \partial J^{a} K^{a} \\
& +\cdots, \\
& \hat{T}(z) J^{b} J^{b} K^{c} K^{c}(w)=-\frac{1}{(z-w)^{4}}\left(N^{2}-1\right)\left[k_{1} K^{a} K^{a}+k_{2} J^{a} J^{a}\right]+\cdots, \\
& \hat{T}(z) J^{b} K^{b} K^{c} K^{c}(w)=-\frac{1}{(z-w)^{4}}\left[2\left(N+k_{2}\right)+\left(N^{2}-1\right) k_{2}\right] J^{a} K^{a}+\cdots, \\
& \hat{T}(z) K^{b} K^{b} K^{c} K^{c}(w)=\left.\hat{T}(z) J^{b} J^{b} J^{c} J^{c}(w)\right|_{k_{1} \leftrightarrow k_{2}, J^{a} \leftrightarrow K^{a}}, \\
& \hat{T}(z) \partial^{2} J^{b} J^{b}(w)=-\frac{1}{(z-w)^{6}} 6 k_{1}\left(N^{2}-1\right)+\frac{1}{(z-w)^{4}} 6 J^{a} J^{a} \\
& +\frac{1}{(z-w)^{3}} 6 \partial J^{a} J^{a}+\cdots, \\
& \hat{T}(z) \partial^{2} J^{b} K^{b}(w)=\frac{1}{(z-w)^{4}} 6 J^{a} K^{a}(w)+\frac{1}{(z-w)^{3}} 6 \partial J^{a} K^{a}+\cdots, \\
& \hat{T}(z) \partial^{2} K^{b} K^{b}(w)=\left.\hat{T}(z) \partial^{2} J^{b} J^{b}(w)\right|_{k_{1} \leftrightarrow k_{2}, J^{a} \leftrightarrow K^{a},} \\
& \hat{T}(z) \partial J^{b} \partial J^{b}(w)=-\frac{1}{(z-w)^{6}} 4 k_{1}\left(N^{2}-1\right)+\frac{1}{(z-w)^{3}} 2 \partial\left(J^{a} J^{a}\right)+\cdots, \\
& \hat{T}(z) \partial J^{b} \partial K^{b}(w)=\frac{1}{(z-w)^{3}} 2\left[J^{a} \partial K^{a}(w)+\partial J^{a} K^{a}\right]+\cdots, \\
& \hat{T}(z) \partial K^{b} \partial K^{c}(w)=\left.\hat{T}(z) \partial J^{b} \partial J^{b}(w)\right|_{k_{1} \leftrightarrow k_{2}, J^{a} \leftrightarrow K^{a},} \\
& \hat{T}(z) J^{b} \partial^{2} K^{b}(w)=\left.\hat{T}(z) \partial^{2} J^{b} K^{b}(w)\right|_{k_{1} \leftrightarrow k_{2}, J^{a} \leftrightarrow K^{a}}
\end{aligned}
$$

According to the operator product expansions (2.17), (2.18), (2.19) and (2.20) and those where the currents $J^{a}(z)$ are replaced by the currents $K^{a}(z)$, one obtains the operator product expansions (E.2)-(E.6) because the operator product expansions of the stress energy tensors $T_{(1)}(z)$ and $T_{(2)}(z)$ with spin-4 currents do not have any higher order singular terms and the currents $J^{a}(z)$ and $K^{a}(z)$ commute with each other. In the operator product expansion (E.7), one uses the relations (C.3) and (C.10) together with the operator product expansion (2.26). By writing the spin 4 current,

$$
\begin{aligned}
d^{a b e} d^{c d e} J^{a} J^{b} J^{c} K^{d}(w) & =d^{a b c} J^{a} Q^{b} K^{c}(w) \\
& -\frac{\left(N^{2}-4\right)}{N} f^{a b c}\left[J^{b} \partial J^{c} K^{a}(w)-\partial J^{b} J^{c} K^{a}(w)\right],
\end{aligned}
$$

in order to compute the operator product expansion (E.8), one need to compute the following operator product expansions

$$
T_{(1)}(z) J^{a} Q^{b}(w)=-\frac{1}{(z-w)^{4}}\left(N+2 k_{1}\right) d^{a b c} J^{c}+\frac{1}{(z-w)^{3}} f^{a b c} Q^{c}
$$




$$
\begin{aligned}
& +\frac{1}{(z-w)^{2}} 3 J^{a} Q^{b}+\frac{1}{(z-w)} \partial\left(J^{a} Q^{b}\right)+\cdots \\
T_{(1)}(z) f^{a b c} \partial J^{b} J^{c}(w) & =\frac{1}{(z-w)^{4}} 4 N J^{a}+\frac{1}{(z-w)^{3}} 2 N \partial J^{a} \\
& +\frac{1}{(z-w)^{2}} 3 f^{a b c} \partial J^{b} J^{c}+\frac{1}{(z-w)} \partial\left(f^{a b c} \partial J^{b} J^{c}\right)+\cdots \\
T_{(1)}(z) f^{a b c} J^{b} \partial J^{c}(w) & =\frac{1}{(z-w)^{4}} 2 N J^{a}+\frac{1}{(z-w)^{3}} 4 N \partial J^{a} \\
& +\frac{1}{(z-w)^{2}} 3 f^{a b c} J^{b} \partial J^{c}+\frac{1}{(z-w)} \partial\left(f^{a b c} J^{b} \partial J^{c}\right)+\cdots
\end{aligned}
$$

In the operator product expansion (E.9), one uses the defining equation (2.10). Due to the different index structures, one cannot obtain the operator product expansion (E.10) directly from the operator product expansion (E.8) via symmetry $\operatorname{arguments}\left(k_{1} \leftrightarrow k_{2}\right.$ and $\left.J^{a} \leftrightarrow K^{a}\right)$. Instead, by using the relation (E.25), one gets the operator product expansion (E.10). From the relations (C.1), (C.8) and (C.10), it is easy to see the operator product expansion (E.12). With the relation (D.32) and the following operator product expansions

$$
\begin{aligned}
T_{(1)}(z) J^{b} T_{(1)}(w) & =\frac{1}{(z-w)^{4}}\left[1+\frac{k_{1}\left(N^{2}-1\right)}{2\left(N+k_{1}\right)}\right] J^{b}+\frac{1}{(z-w)^{2}} 3 J^{b} T_{(1)}+\frac{1}{(z-w)} \partial\left(J^{b} T_{(1)}\right) \\
& +\cdots, \\
T_{(1)}(z) \partial^{2} J^{b}(w) & =\frac{1}{(z-w)^{4}} 6 J^{b}+\frac{1}{(z-w)^{3}} 6 \partial J^{b}+\frac{1}{(z-w)^{2}} 3 \partial^{2} J^{b}+\frac{1}{(z-w)} \partial^{3} J^{b} \\
& +\cdots,
\end{aligned}
$$

together with the operator product expansion (E.26), one can check the relation (E.13). The operator product expansion (E.29) can be obtained by adding the operator product expansions (E.26) and (E.27). Of course, one also gets the operator product expansion

$$
\begin{aligned}
T_{(1)}(z) J^{a} J^{a} J^{b}(w) & =-\frac{1}{(z-w)^{4}} k_{1}\left(N^{2}+1\right) J^{b}-\frac{1}{(z-w)^{3}} 2 N \partial J^{b} \\
& +\frac{1}{(z-w)^{2}} 3 J^{a} J^{a} J^{b}+\frac{1}{(z-w)} \partial\left(J^{a} J^{a} J^{b}\right)+\cdots
\end{aligned}
$$

This operator product expansion (E.30) is also useful to check the operator product expansion (E.13). For the operator product expansion (E.14), one uses the defining equation (2.3). For the operator product expansion (E.15), the relation (E.28) is used. In order to compute the operator product expansion (E.17), one can use the relation (C.10). The relation (E.29) gives the result of (E.18). By using the identities (C.2), (C.9) and (C.10), one gets the operator product expansion (E.20). The remaining operator product expansions can be obtained similarly. 


\section{Appendix F The coefficient functions that satisfy the lin- ear equations in subsection 3.2}

By collecting the $\frac{1}{(z-w)^{2}}$ terms in the operator product expansions (D.1) $-(\bar{D} .22)$ we have eighteen equations for twenty two unknown coefficient functions.

$$
\begin{aligned}
& {\left[-12\left(N+k_{1}\right) c_{1}-3 k_{2} c_{2}-4\left(N+k_{1}\right) c_{6}-k_{2} c_{7}\right] d^{a b c} J^{b} Q^{c}=0,} \\
& {\left[\frac{48\left(N^{2}-4\right)\left(N+k_{1}\right)}{N\left(N^{2}+1\right)} c_{1}+\frac{12 k_{2}\left(N^{2}-4\right)}{N\left(N^{2}+1\right)} c_{2}-4\left(N+k_{1}\right) c_{11}-k_{2} c_{12}\right] J^{a} J^{b} J^{b}=0 \text {, }} \\
& {\left[\frac{2\left(N^{2}-4\right)}{N\left(N^{2}+1\right)}\left(3 N\left(N^{2}-5\right)+2 k_{1}\left(N^{2}-3\right)\right) c_{1}+\frac{k_{2}\left(N^{2}-4\right)\left(N^{2}-3\right)}{N\left(N^{2}+1\right)} c_{2}+\frac{\left(N^{2}-4\right)\left(N+2 k_{1}\right)}{N} c_{6}\right.} \\
& \left.+\frac{\left(N^{2}-4\right) k_{2}}{N} c_{7}+2\left(N+k_{1}\right) c_{11}+k_{2} c_{12}-\frac{k_{1}}{N} c_{16}-\frac{k_{2}}{N} c_{17}-c_{19}\right] f^{a b c} J^{b} \partial J^{c}=0, \\
& {\left[-\frac{2\left(N^{2}-4\right)}{N\left(N^{2}+1\right)}\left(N\left(5 N^{2}-21\right)+4 k_{1}\left(N^{2}-3\right)\right) c_{1}-\frac{2 k_{2}\left(N^{2}-4\right)\left(N^{2}-3\right)}{N\left(N^{2}+1\right)} c_{2}\right.} \\
& -\frac{\left(N^{2}-4\right)\left(N+2 k_{1}\right)}{N} c_{6} \\
& \left.-\frac{\left(N^{2}-4\right) k_{2}}{N} c_{7}-2\left(N+k_{1}\right) c_{11}-k_{2} c_{12}-\left(2+\frac{k_{1}}{N}\right) c_{16}-\frac{k_{2}}{N} c_{17}+c_{19}\right] f^{a b c} \partial J^{b} J^{c}=0, \\
& {\left[\left(-3 k_{1}-\frac{12\left(2 N^{2}-3\right)}{N\left(N^{2}+1\right)}\right) c_{2}+\left(-2 k_{2}-\frac{2\left(N^{2}-4\right)\left(N^{2}-3\right)}{N\left(N^{2}+1\right)}\right) c_{3}+\left(-\left(N+k_{1}\right)+\frac{2\left(N^{2}-4\right)}{N}\right) c_{7}\right.} \\
& \left.-\left(N+2 k_{2}\right) c_{8}+2 c_{12}\right] d^{a b c} Q^{b} K^{c}=0 \\
& {\left[-\frac{\left(N^{2}-4\right)}{\left(N^{2}+1\right)}\left(\frac{3 k_{1}\left(N^{2}-3\right)}{N}+4\left(N^{2}-6\right)\right) c_{2}-\frac{2\left(N^{2}-4\right)}{\left(N^{2}+1\right)}\left(\frac{k_{2}}{N}\left(N^{2}-3\right)+2\right) c_{3}\right.} \\
& \left.-\left(N^{2}-4\right) c_{7}-2 N c_{12}-2 c_{17}+c_{20}\right] f^{a b c} \partial J^{b} K^{c}=0 \\
& {\left[\left(-6 k_{1}-\frac{6\left(N^{4}-3 N^{2}+6\right)}{N\left(N^{2}+1\right)}\right) c_{2}+\left(-\frac{8\left(2 N^{2}-3\right)}{N\left(N^{2}+1\right)}-4 k_{2}\right) c_{3}+\left(-\left(N+2 k_{1}\right)-\frac{2}{N}\left(N^{2}-4\right)\right) c_{7}\right.} \\
& \left.-2 c_{12}\right] d^{a c e} d^{b d e} J^{c} J^{d} K^{b}=0 \text {, } \\
& {\left[\frac{12\left(N^{2}-4\right)}{N\left(N^{2}+1\right)}\left(2 k_{1}+\frac{N^{2}+3}{N}\right) c_{2}+\frac{8\left(N^{2}-4\right)}{N\left(N^{2}+1\right)}\left(2 k_{2}+\frac{N^{2}-3}{N}\right) c_{3}\right.} \\
& \left.-\frac{8}{N^{2}}\left(N^{2}-4\right) c_{7}+\left(-2\left(N+k_{1}\right)-\frac{8}{N}\right) c_{12}\right] J^{a} J^{b} K^{b}=0, \\
& {\left[\frac{12\left(N^{2}-4\right)}{N\left(N^{2}+1\right)}\left(k_{1}+\frac{N^{2}-3}{N}\right) c_{2}+\frac{8\left(N^{2}-4\right)}{N\left(N^{2}+1\right)}\left(k_{2}+\frac{3}{N}\right) c_{3}+\frac{8\left(N^{2}-4\right)}{N^{2}} c_{7}+\left(-k_{1}+\frac{8}{N}\right) c_{12}\right.} \\
& \left.-2\left(N+k_{2}\right) c_{13}\right] J^{b} J^{b} K^{a}=0, \\
& {\left[\left(-2 k_{1}-\frac{2\left(N^{2}-4\right)\left(N^{2}-3\right)}{N\left(N^{2}+1\right)}\right) c_{3}+\left(-3 k_{2}-\frac{12\left(2 N^{2}-3\right)}{N\left(N^{2}+1\right)}\right) c_{4}-\left(N+2 k_{1}\right) c_{8}\right.} \\
& \left.-\left(N+k_{2}\right) c_{9}\right] d^{a b c} J^{b} R^{c}=0,
\end{aligned}
$$




$$
\begin{aligned}
& {\left[\frac{2\left(N^{2}-4\right)}{N^{2}+1}\left(2+\frac{k_{1}}{N}\left(N^{2}-3\right)\right) c_{3}+\frac{\left(N^{2}-4\right)}{\left(N^{2}+1\right)}\left(\frac{3 k_{2}\left(N^{2}-3\right)}{N}+4\left(N^{2}-6\right)\right) c_{4}\right.} \\
& \left.+\frac{\left(N+2 k_{2}\right)\left(N^{2}-4\right)}{N} c_{9}+2\left(N+k_{2}\right) c_{14}-c_{20}+2 c_{22}\right] f^{a b c} J^{b} \partial K^{c}=0 \\
& {\left[\left(-4 k_{1}-\frac{8\left(2 N^{2}-3\right)}{N\left(N^{2}+1\right)}\right) c_{3}+\left(-6 k_{2}-\frac{6\left(N^{4}-3 N^{2}+6\right)}{N\left(N^{2}+1\right)}\right) c_{4}-\left(N+2 k_{2}\right) c_{9}\right] d^{a c e} d^{b d e} J^{b} K^{c} K^{d}=0,} \\
& {\left[\left(\frac{8\left(N^{2}-4\right)}{N\left(N^{2}+1\right)}\left(2 k_{1}+\frac{N^{2}-3}{N}\right) c_{3}+\frac{12\left(N^{2}-4\right)}{N\left(N^{2}+1\right)}\left(2 k_{2}+\frac{N^{2}+3}{N}\right) c_{4}-2\left(N+k_{2}\right) c_{14}\right] J^{b} K^{a} K^{b}=0,\right.} \\
& {\left[\frac{8\left(N^{2}-4\right)}{N\left(N^{2}+1\right)}\left(k_{1}+\frac{3}{N}\right) c_{3}+\frac{12\left(N^{2}-4\right)}{N\left(N^{2}+1\right)}\left(k_{2}+\frac{N^{2}-3}{N}\right) c_{4}-2\left(N+k_{1}\right) c_{13}-k_{2} c_{14}\right] J^{a} K^{b} K^{b}=0,} \\
& {\left[-3 k_{1} c_{4}-12\left(N+k_{2}\right) c_{5}-k_{1} c_{9}-4\left(N+k_{2}\right) c_{10}\right] d^{a b c} K^{b} R^{c}=0,} \\
& {\left[\frac{12 k_{1}\left(N^{2}-4\right)}{N\left(N^{2}+1\right)} c_{4}+\frac{48\left(N^{2}-4\right)\left(N+k_{2}\right)}{N\left(N^{2}+1\right)} c_{5}-k_{1} c_{14}-4\left(N+k_{2}\right) c_{15}\right] K^{a} K^{b} K^{b}=0,} \\
& {\left[\frac{k_{1}\left(N^{2}-4\right)\left(N^{2}-3\right)}{N\left(N^{2}+1\right)} c_{4}+\frac{2\left(N^{2}-4\right)}{N\left(N^{2}+1\right)}\left(3 N\left(N^{2}-5\right)+2 k_{2}\left(N^{2}-3\right)\right) c_{5}\right.} \\
& \left.+\frac{\left(N^{2}-4\right)\left(N+2 k_{2}\right)}{N} c_{10}+2\left(N+k_{2}\right) c_{15}-\frac{k_{2}}{N} c_{18}-c_{21}-\frac{k_{1}}{N} c_{22}\right] f^{a b c} K^{b} \partial K^{c}=0, \\
& {\left[-\frac{2 k_{1}\left(N^{2}-4\right)\left(N^{2}-3\right)}{N\left(N^{2}+1\right)} c_{4}-\frac{2\left(N^{2}-4\right)}{N\left(N^{2}+1\right)}\left(N\left(5 N^{2}-21\right)+4 k_{2}\left(N^{2}-3\right)\right) c_{5}\right.} \\
& -\frac{\left.N^{2}-4\right)\left(N+2 k_{2}\right)}{N} c_{10}-2\left(N+k_{2}\right) c_{15}-\left(2+c_{18}+c_{21}-\frac{k_{1}}{N} c_{22}\right] f^{a b c} \partial K^{b} K^{c}=0 .
\end{aligned}
$$

Therefore, there exist the following independent terms

$$
\begin{aligned}
& d^{a b c} J^{b} Q^{c}(w), J^{a} J^{b} J^{b}(w), f^{a b c} J^{b} \partial J^{c}(w), f^{a b c} \partial J^{b} J^{c}(w), d^{a b c} Q^{b} K^{c}(w), f^{a b c} \partial J^{b} K^{c}(w), \\
& d^{a c e} d^{b d e} J^{c} J^{d} K^{b}(w), J^{a} J^{b} K^{b}(w), J^{b} J^{b} K^{a}(w), d^{a b c} J^{b} R^{c}(w), f^{a b c} J^{b} \partial K^{c}(w), \\
& d^{a c e} d^{b d e} J^{b} K^{c} K^{d}(w), J^{b} K^{a} K^{b}(w), J^{a} K^{b} K^{b}(w), d^{a b c} K^{b} R^{c}(w), K^{a} K^{b} K^{b}(w), \\
& f^{a b c} K^{b} \partial K^{c}(w), f^{a b c} \partial K^{b} K^{c}(w) .
\end{aligned}
$$

The $\frac{1}{(z-w)^{3}}$ terms in the operator product expansions (D.1)-(D.22) provide three equations for the coefficient functions

$$
\begin{aligned}
& {\left[-\frac{4 N\left(N^{2}-4\right)\left(N^{2}-9\right)}{\left(N^{2}+1\right)} c_{1}-2\left(N^{2}-4\right)\left(N+2 k_{1}\right) c_{6}-4 N\left(N+k_{1}\right) c_{11}-2 N c_{16}\right.} \\
& \left.-2\left(N+2 k_{1}\right) c_{19}-2 k_{2} c_{20}\right] \partial J^{a}=0, \\
& {\left[-\frac{4 N\left(N^{2}-4\right)\left(N^{2}-9\right)}{\left(N^{2}+1\right)} c_{5}-2\left(N^{2}-4\right)\left(N+2 k_{2}\right) c_{10}-4 N\left(N+k_{2}\right) c_{15}-2 N c_{18}\right.} \\
& \left.-2 k_{1} c_{20}-2\left(N+2 k_{2}\right) c_{21}\right] \partial K^{a}=0, \\
& {\left[\frac{2\left(N^{2}-4\right)\left(N^{2}-9\right)}{\left(N^{2}+1\right)} c_{2}-\frac{2\left(N^{2}-4\right)\left(N^{2}-9\right)}{\left(N^{2}+1\right)} c_{4}+\left(1-\frac{2 k_{1}}{N}\right)\left(N^{2}-4\right) c_{7}-\frac{N^{2}-4}{N}\left(N+2 k_{2}\right) c_{9}\right.}
\end{aligned}
$$




$$
\left.-2\left(k_{1}-N\right) c_{12}-2\left(N+k_{2}\right) c_{14}-2 c_{17}+2 c_{22}\right] f^{a b c} J^{b} K^{c}=0 .
$$

The $\frac{1}{(z-w)^{4}}$ terms in the operator product expansions (D.1)-(D.22) provide two equations for the coefficient functions

$$
\begin{aligned}
& {\left[\frac{4 N\left(N^{2}-4\right)\left(N^{2}-9\right)}{\left(N^{2}+1\right)} c_{1}+2\left(N^{2}-4\right)\left(N+2 k_{1}\right) c_{6}+4 N\left(N+k_{1}\right) c_{11}-2\left(2 N+3 k_{1}\right) c_{16}\right.} \\
& \left.-2 N c_{19}-6 k_{2} c_{22}\right] J^{a}=0 \\
& {\left[\frac{4 N\left(N^{2}-4\right)\left(N^{2}-9\right)}{\left(N^{2}+1\right)} c_{5}+2 k_{1}\left(N^{2}-4\right) c_{7}+2\left(N^{2}-4\right)\left(N+2 k_{2}\right) c_{10}+2 N k_{1} c_{12}\right.} \\
& \left.+4 N\left(N+k_{2}\right) c_{15}-6 k_{1} c_{17}-2\left(2 N+3 k_{2}\right) c_{18}-2 N c_{21}\right] K^{a}=0 .
\end{aligned}
$$

On the other hands, the $\frac{1}{(z-w)^{3}}$ terms in the operator product expansions (E.2) $-(\overline{E .23})$ lead to the following equations:

$$
\begin{aligned}
& {\left[-2\left(N^{2}-4\right) c_{7}+2 c_{20}-2 N c_{12}+6 c_{17}\right] \partial J^{a} K^{a}=0,} \\
& {\left[6 c_{16}+4 c_{19}\right] \partial J^{a} J^{a}=0,} \\
& {\left[6 c_{18}+4 c_{21}\right] \partial K^{a} K^{a}=0,} \\
& {\left[2 c_{20}+6 c_{22}\right] J^{a} \partial K^{a}=0 .}
\end{aligned}
$$

The $\frac{1}{(z-w)^{4}}$ terms in the operator product expansions (E.2) $-(\overline{E .23})$ lead to the following equations:

$$
\begin{aligned}
& {\left[-\frac{4\left(N^{2}-4\right)\left(N+2 k_{1}\right)}{N} c_{6}-2\left[2\left(N+k_{1}\right)+k_{1}\left(N^{2}-1\right)\right] c_{11}-k_{2}\left(N^{2}-1\right) c_{13}+6 c_{16}\right] J^{a} J^{a}=0,} \\
& {\left[-\frac{4 k_{1}\left(N^{2}-4\right)}{N} c_{7}-\frac{2\left(N^{2}-4\right)\left(N+2 k_{2}\right)}{N} c_{9}-k_{1}\left(N^{2}+1\right) c_{12}\right.} \\
& \left.+\left(-2\left(N+k_{2}\right)-k_{2}\left(N^{2}-1\right)\right) c_{14}+6 c_{17}+6 c_{22}\right] J^{a} K^{a}=0, \\
& {\left[-\frac{4\left(N^{2}-4\right)\left(N+2 k_{2}\right)}{N} c_{10}-k_{1}\left(N^{2}-1\right) c_{13}-2\left(2\left(N+k_{2}\right)+k_{2}\left(N^{2}-1\right)\right) c_{15}\right.} \\
& \left.+6 c_{18}\right] K^{a} K^{a}=0 .
\end{aligned}
$$

Finally, the $\frac{1}{(z-w)^{6}}$ terms in the operator product expansions $(\overline{E .2})-(\overline{E .23})$ provide the relation

$$
\left[-6 k_{1}\left(N^{2}-1\right) c_{16}-6 k_{2}\left(N^{2}-1\right) c_{18}-4 k_{1}\left(N^{2}-1\right) c_{19}-4 k_{2}\left(N^{2}-1\right) c_{21}\right]=0 .
$$

Then it is easy to see that $c_{16}=0=c_{18}$ from the relation (F.5) by using the conditions $c_{19}=0=c_{21}$ which was shown in subsection 3.2 . Then the equation (F.7) is automatically satisfied. Now we are left with 18 equations in the relations (F.1), 3 equations in the relations 
(F.3), 2 equations in the relations (F.4 $), 2$ equations in the relations (F.5) and 3 equations in the relations (F.6). Totally, there are 28 equations we have to solve.

The coefficients satisfying the equations (F.1)-(F.7) are fixed and they can be written in terms of $c_{1}$

$$
\begin{aligned}
& c_{2}=\frac{-4 N\left(N+k_{1}\right)}{3 k_{2}\left(N+2 k_{1}\right) D\left(k_{1}, k_{2}, N\right)}\left[90 k_{1}^{3}+270 k_{1}^{2} k_{2}+180 k_{1} k_{2}^{2}+255 k_{1}^{2} N+363 k_{1} k_{2} N\right. \\
& \text { - } 72 k_{2}^{2} N-36 k_{1}^{2} k_{2}^{2} N+48 k_{1} N^{2}-100 k_{1}^{3} N^{2}-102 k_{2} N^{2}-332 k_{1}^{2} k_{2} N^{2} \\
& \text { - } 202 k_{1} k_{2}^{2} N^{2}-60 N^{3}-266 k_{1}^{2} N^{3}-478 k_{1} k_{2} N^{3}+52 k_{2}^{2} N^{3}+12 k_{1}^{2} k_{2}^{2} N^{3} \\
& \text { - } 106 k_{1} N^{4}+10 k_{1}^{3} N^{4}+84 k_{2} N^{4}+62 k_{1}^{2} k_{2} N^{4}+50 k_{1} k_{2}^{2} N^{4}+72 N^{5} \\
& \left.+35 k_{1}^{2} N^{5}+115 k_{1} k_{2} N^{5}+8 k_{2}^{2} N^{5}+30 k_{1} N^{6}+18 k_{2} N^{6}\right] c_{1} \text {, } \\
& c_{3}=\frac{2 N\left(N+k_{1}\right)\left(2 N+3 k_{1}\right)\left(N^{2}-3\right)}{k_{2} D\left(k_{1}, k_{2}, N\right)}\left[-5 k_{1}-5 k_{2}-10 N+2 k_{1} k_{2} N+7 k_{1} N^{2}+7 k_{2} N^{2}\right. \\
& \left.+12 N^{3}\right] c_{1} \\
& c_{4}=\frac{4 N\left(N+k_{1}\right)\left(2 N+3 k_{1}\right)}{3 k_{2}\left(2 N+3 k_{2}\right) D\left(k_{1}, k_{2}, N\right)}\left[45 k_{1} k_{2}+45 k_{2}^{2}+30 k_{1} N+120 k_{2} N+18 k_{1}^{2} k_{2} N+60 N^{2}\right. \\
& +28 k_{1}^{2} N^{2}-34 k_{1} k_{2} N^{2}-50 k_{2}^{2} N^{2}-4 k_{1} N^{3}-142 k_{2} N^{3}-6 k_{1}^{2} k_{2} N^{3} \\
& \text { - } \left.72 N^{4}-16 k_{1}^{2} N^{4}-11 k_{1} k_{2} N^{4}+5 k_{2}^{2} N^{4}-26 k_{1} N^{5}+10 k_{2} N^{5}\right] c_{1} \text {, } \\
& c_{5}=\frac{k_{1}\left(N+k_{1}\right)\left(2 N+3 k_{1}\right)}{k_{2}\left(N+k_{2}\right)\left(2 N+3 k_{2}\right) D\left(k_{1}, k_{2}, N\right)}\left[-18 k_{1}^{2} k_{2}-36 k_{1} k_{2}^{2}-18 k_{2}^{3}-12 k_{1}^{2} N-75 k_{1} k_{2} N\right. \\
& \text { - } 63 k_{2}^{2} N-34 k_{1} N^{2}-64 k_{2} N^{2}+14 k_{1}^{2} k_{2} N^{2}+40 k_{1} k_{2}^{2} N^{2}+20 k_{2}^{3} N^{2}-20 N^{3}+4 k_{1}^{2} N^{3} \\
& +78 k_{1} k_{2} N^{3}+70 k_{2}^{2} N^{3}+28 k_{1} N^{4}+74 k_{2} N^{4}-4 k_{1} k_{2}^{2} N^{4}-2 k_{2}^{3} N^{4} \\
& \left.+24 N^{5}+4 k_{1}^{2} N^{5}-3 k_{1} k_{2} N^{5}-7 k_{2}^{2} N^{5}+6 k_{1} N^{6}-6 k_{2} N^{6}\right] c_{1} \text {, } \\
& c_{6}=\frac{2\left(N^{2}-9\right)}{\left(N+2 k_{1}\right)\left(N^{2}+1\right) D\left(k_{1}, k_{2}, N\right)}\left[-6 k_{1}^{4}-12 k_{1}^{3} k_{2}-6 k_{1}^{2} k_{2}^{2}-24 k_{1}^{3} N-48 k_{1}^{2} k_{2} N\right. \\
& \text { - } 24 k_{1} k_{2}^{2} N-53 k_{1}^{2} N^{2}-46 k_{2} N^{3}+12 k_{1}^{2} k_{2} N^{3}+10 k_{1} k_{2}^{2} N^{3}-20 N^{4}+22 k_{1}^{2} N^{4} \\
& +6 k_{1}^{4} N^{4}+46 k_{1} k_{2} N^{4}+12 k_{1}^{3} k_{2} N^{4}+20 k_{2}^{2} N^{4}+6 k_{1}^{2} k_{2}^{2} N^{4}+46 k_{1} N^{5} \\
& +24 k_{1}^{3} N^{5}+44 k_{2} N^{5}+36 k_{1}^{2} k_{2} N^{5}+10 k_{1} k_{2}^{2} N^{5}+24 N^{6}+31 k_{1}^{2} N^{6} \\
& \left.+31 k_{1} k_{2} N^{6}+14 k_{1} N^{7}+2 k_{2} N^{7}-77 k_{1} k_{2} N^{2}-24 k_{2}^{2} N^{2}-56 k_{1} N^{3}\right] c_{1}, \\
& c_{7}=-\frac{8 N\left(N^{2}-9\right)\left(N+k_{1}\right)}{k_{2}\left(N+2 k_{1}\right)\left(N^{2}+1\right) D\left(k_{1}, k_{2}, N\right)}\left[5 k_{1}^{3}-2 k_{1}^{2} k_{2}-7 k_{1} k_{2}^{2}-7 k_{1}^{2} N-33 k_{1} k_{2} N\right. \\
& \text { - } 26 k_{2}^{2} N-36 k_{1} N^{2}-46 k_{2} N^{2}+12 k_{1}^{2} k_{2} N^{2}+14 k_{1} k_{2}^{2} N^{2}-20 N^{3}+22 k_{1}^{2} N^{3}+46 k_{1} k_{2} N^{3} \\
& +20 k_{2}^{2} N^{3}+42 k_{1} N^{4}-5 k_{1}^{3} N^{4}+44 k_{2} N^{4}-10 k_{1}^{2} k_{2} N^{4}-3 k_{1} k_{2}^{2} N^{4}+24 N^{5}-15 k_{1}^{2} N^{5} \\
& \left.-13 k_{1} k_{2} N^{5}+2 k_{2}^{2} N^{5}-10 k_{1} N^{6}+2 k_{2} N^{6}\right] c_{1},
\end{aligned}
$$




$$
\begin{aligned}
& c_{8}=-\frac{4 N\left(N^{2}-9\right)\left(N+k_{1}\right)\left(2 N+3 k_{1}\right)\left(N^{2}-3\right)}{k_{2}\left(N+2 k_{1}\right)\left(N+2 k_{2}\right)\left(N^{2}+1\right) D\left(k_{1}, k_{2}, N\right)}\left[-8 k_{1}^{2} k_{2}-8 k_{1} k_{2}^{2}-13 k_{1}^{2} N\right. \\
& -26 k_{1} k_{2} N-13 k_{2}^{2} N-23 k_{1} N^{2}-23 k_{2} N^{2}+4 k_{1}^{2} k_{2} N^{2}+4 k_{1} k_{2}^{2} N^{2}-10 N^{3}+9 k_{1}^{2} N^{3} \\
& \left.+20 k_{1} k_{2} N^{3}+9 k_{2}^{2} N^{3}+21 k_{1} N^{4}+21 k_{2} N^{4}+12 N^{5}\right] c_{1} \text {, } \\
& c_{9}=-\frac{8 N\left(N^{2}-9\right)\left(N+k_{1}\right)\left(2 N+3 k_{1}\right)}{k_{2}\left(N+2 k_{2}\right)\left(2 N+3 k_{2}\right)\left(N^{2}+1\right) D\left(k_{1}, k_{2}, N\right)}\left[-17 k_{1}^{2} k_{2}-22 k_{1} k_{2}^{2}-5 k_{2}^{3}-22 k_{1}^{2} N\right. \\
& \text { - } 59 k_{1} k_{2} N-37 k_{2}^{2} N-42 k_{1} N^{2}-52 k_{2} N^{2}+10 k_{1}^{2} k_{2} N^{2}+12 k_{1} k_{2}^{2} N^{2}-20 N^{3}+20 k_{1}^{2} N^{3} \\
& +46 k_{1} k_{2} N^{3}+22 k_{2}^{2} N^{3}+44 k_{1} N^{4}+46 k_{2} N^{4}+3 k_{1}^{2} k_{2} N^{4}+10 k_{1} k_{2}^{2} N^{4}+5 k_{2}^{3} N^{4} \\
& \left.+24 N^{5}-2 k_{1}^{2} N^{5}+13 k_{1} k_{2} N^{5}+15 k_{2}^{2} N^{5}-2 k_{1} N^{6}+10 k_{2} N^{6}\right] c_{1} \\
& c_{10}=\frac{2 k_{1}\left(N^{2}-9\right)\left(N+k_{1}\right)\left(2 N+3 k_{1}\right)}{k_{2}\left(N+k_{2}\right)\left(N+2 k_{2}\right)\left(2 N+3 k_{2}\right)\left(N^{2}+1\right) D\left(k_{1}, k_{2}, N\right)}\left[-6 k_{1}^{2} k_{2}^{2}-12 k_{1} k_{2}^{3}-6 k_{2}^{4}\right. \\
& \text { - } 24 k_{1}^{2} k_{2} N-48 k_{1} k_{2}^{2} N-24 k_{2}^{3} N-24 k_{1}^{2} N^{2}-77 k_{1} k_{2} N^{2}-53 k_{2}^{2} N^{2}-46 k_{1} N^{3} \\
& \text { - } 56 k_{2} N^{3}+10 k_{1}^{2} k_{2} N^{3}+12 k_{1} k_{2}^{2} N^{3}-20 N^{4}+20 k_{1}^{2} N^{4}+46 k_{1} k_{2} N^{4}+22 k_{2}^{2} N^{4} \\
& +6 k_{1}^{2} k_{2}^{2} N^{4}+12 k_{1} k_{2}^{3} N^{4}+6 k_{2}^{4} N^{4}+44 k_{1} N^{5}+46 k_{2} N^{5}+10 k_{1}^{2} k_{2} N^{5}+36 k_{1} k_{2}^{2} N^{5} \\
& \left.+24 k_{2}^{3} N^{5}+24 N^{6}+31 k_{1} k_{2} N^{6}+31 k_{2}^{2} N^{6}+2 k_{1} N^{7}+14 k_{2} N^{7}\right] c_{1}, \\
& c_{11}=-\frac{4\left(N^{2}-4\right)\left(N^{2}-9\right)}{N\left(N^{2}+1\right) D\left(k_{1}, k_{2}, N\right)}\left[-6 k_{1}^{3}-12 k_{1}^{2} k_{2}-6 k_{1} k_{2}^{2}-21 k_{1}^{2} N-33 k_{1} k_{2} N\right. \\
& +6 k_{1}^{3} N^{2}-26 k_{2} N^{2}+12 k_{1}^{2} k_{2} N^{2}+6 k_{1} k_{2}^{2} N^{2}-10 N^{3}+21 k_{1}^{2} N^{3}+33 k_{1} k_{2} N^{3} \\
& \left.+10 k_{2}^{2} N^{3}+26 k_{1} N^{4}+26 k_{2} N^{4}+12 N^{5}-12 k_{2}^{2} N-26 k_{1} N^{2}\right] c_{1} \text {, } \\
& c_{12}=\frac{16\left(N^{2}-4\right)\left(N^{2}-9\right)\left(N+k_{1}\right)}{k_{2}\left(N+2 k_{1}\right)\left(N^{2}+1\right) D\left(k_{1}, k_{2}, N\right)}\left[10 k_{1}^{3}+14 k_{1}^{2} k_{2}+4 k_{1} k_{2}^{2}+19 k_{1}^{2} N+3 k_{1} k_{2} N\right. \\
& \text { - } 6 k_{1} N^{2}-10 k_{1}^{3} N^{2}-26 k_{2} N^{2}-14 k_{1}^{2} k_{2} N^{2}-10 N^{3}-19 k_{1}^{2} N^{3}-3 k_{1} k_{2} N^{3} \\
& \left.+14 k_{2}^{2} N^{3}+2 k_{1} N^{4}+26 k_{2} N^{4}+12 N^{5}-16 k_{2}^{2} N\right] c_{1} \\
& c_{13}=-\frac{8\left(k_{1}+k_{2}\right)\left(N^{2}-4\right)\left(N^{2}-9\right)\left(N+k_{1}\right)\left(2 N+3 k_{1}\right)\left(N^{2}-3\right)}{k_{2}\left(N^{2}+1\right) D\left(k_{1}, k_{2}, N\right)} c_{1}, \\
& c_{14}=\frac{16\left(N^{2}-4\right)\left(N^{2}-9\right)\left(N+k_{1}\right)\left(2 N+3 k_{1}\right)}{k_{2}\left(2 N+3 k_{2}\right)\left(N^{2}+1\right) D\left(k_{1}, k_{2}, N\right)}\left[-8 k_{1}^{2}-13 k_{1} k_{2}-5 k_{2}^{2}-18 k_{1} N-18 k_{2} N\right. \\
& \left.-10 N^{2}+6 k_{1}^{2} N^{2}+13 k_{1} k_{2} N^{2}+5 k_{2}^{2} N^{2}+18 k_{1} N^{3}+18 k_{2} N^{3}+12 N^{4}\right] c_{1}, \\
& c_{15}=-\frac{4 k_{1}\left(N^{2}-4\right)\left(N^{2}-9\right)\left(N+k_{1}\right)\left(2 N+3 k_{1}\right)}{k_{2} N\left(N+k_{2}\right)\left(2 N+3 k_{2}\right)\left(N^{2}+1\right) D\left(k_{1}, k_{2}, N\right)}\left[-6 k_{1}^{2} k_{2}-12 k_{1} k_{2}^{2}-6 k_{2}^{3}-12 k_{1}^{2} N\right. \\
& -33 k_{1} k_{2} N-21 k_{2}^{2} N-26 k_{1} N^{2}-26 k_{2} N^{2}+6 k_{1}^{2} k_{2} N^{2}+12 k_{1} k_{2}^{2} N^{2}+6 k_{2}^{3} N^{2} \\
& \left.-10 N^{3}+10 k_{1}^{2} N^{3}+33 k_{1} k_{2} N^{3}+21 k_{2}^{2} N^{3}+26 k_{1} N^{4}+26 k_{2} N^{4}+12 N^{5}\right] c_{1}, \\
& c_{16}=0
\end{aligned}
$$




$$
\begin{aligned}
c_{17} & =-\frac{4\left(N^{2}-4\right)\left(N^{2}-9\right)\left(N+k_{1}\right)\left(N^{2}-3\right)}{3 k_{2}\left(N+2 k_{1}\right)\left(N^{2}+1\right) D\left(k_{1}, k_{2}, N\right)}\left[6 k_{1}^{4}+12 k_{1}^{3} k_{2}+6 k_{1}^{2} k_{2}^{2}+29 k_{1}^{3} N+46 k_{1}^{2} k_{2} N\right. \\
& +17 k_{1} k_{2}^{2} N+46 k_{1}^{2} N^{2}-6 k_{1}^{4} N^{2}+44 k_{1} k_{2} N^{2}-12 k_{1}^{3} k_{2} N^{2}-2 k_{2}^{2} N^{2}-6 k_{1}^{2} k_{2}^{2} N^{2} \\
& +20 k_{1} N^{3}-29 k_{1}^{3} N^{3}-46 k_{1}^{2} k_{2} N^{3}-13 k_{1} k_{2}^{2} N^{3}-46 k_{1}^{2} N^{4}-44 k_{1} k_{2} N^{4}+2 k_{2}^{2} N^{4} \\
& \left.-24 k_{1} N^{5}\right] c_{1}, \\
c_{18} & =0, \\
c_{19} & =0, \\
c_{20} & =-\frac{4\left(k_{1}+k_{2}\right)\left(N^{2}-1\right)\left(N^{2}-4\right)\left(N^{2}-9\right)\left(N+k_{1}\right)\left(2 N+3 k_{1}\right)\left(2 N+k_{1}+k_{2}\right)\left(N^{2}-3\right)}{k_{2}\left(N^{2}+1\right) D\left(k_{1}, k_{2}, N\right)} c_{1}, \\
c_{21} & =0, \\
c_{22} & =\frac{4\left(k_{1}+k_{2}\right)\left(N^{2}-1\right)\left(N^{2}-4\right)\left(N^{2}-9\right)\left(N+k_{1}\right)\left(2 N+3 k_{1}\right)\left(2 N+k_{1}+k_{2}\right)\left(N^{2}-3\right)}{3 k_{2}\left(N^{2}+1\right) D\left(k_{1}, k_{2}, N\right)} c_{1},
\end{aligned}
$$

where we introduce the function $D\left(k_{1}, k_{2}, N\right)$ which depends on the levels $k_{1}, k_{2}$ and $N 15$

$$
\begin{aligned}
D\left(k_{1}, k_{2}, N\right) & \equiv-18 k_{1}^{3}-36 k_{1}^{2} k_{2}-18 k_{1} k_{2}^{2}-63 k_{1}^{2} N-75 k_{1} k_{2} N-12 k_{2}^{2} N-64 k_{1} N^{2} \\
& +20 k_{1}^{3} N^{2}-34 k_{2} N^{2}+40 k_{1}^{2} k_{2} N^{2}+14 k_{1} k_{2}^{2} N^{2}-20 N^{3}+70 k_{1}^{2} N^{3} \\
& +78 k_{1} k_{2} N^{3}+4 k_{2}^{2} N^{3}+74 k_{1} N^{4}-2 k_{1}^{3} N^{4}+28 k_{2} N^{4}-4 k_{1}^{2} k_{2} N^{4}+24 N^{5} \\
& -7 k_{1}^{2} N^{5}-3 k_{1} k_{2} N^{5}+4 k_{2}^{2} N^{5}-6 k_{1} N^{6}+6 k_{2} N^{6} .
\end{aligned}
$$

Note that for $N=3$, the coefficients $c_{6}, \cdots, c_{22}$, that have the factor $\left(N^{2}-9\right)$, vanish. Also we present the large $N$ limit (3.19) for these coefficients as follows:

$$
\begin{aligned}
c_{2} & =\left[\frac{4 \lambda(4+5 \lambda)}{3(-1+\lambda)(2+\lambda)}\right] c_{1}, \quad c_{3}=\left[\frac{2 \lambda^{2}(7+5 \lambda)}{(-1+\lambda)^{2}(2+\lambda)}\right] c_{1}, \\
c_{4} & =\left[\frac{20 \lambda^{2}(1+\lambda)}{3(-3+\lambda)(-1+\lambda)(2+\lambda)}\right] c_{1}, \quad c_{5}=-\left[\frac{\lambda^{2}(1+\lambda)}{N(-3+\lambda)(-1+\lambda)}\right] c_{1}, \\
c_{6} & =\left[\frac{2 \lambda}{2+\lambda}\right] c_{1}, \quad c_{7}=\left[\frac{8 \lambda}{(-1+\lambda)(2+\lambda)}\right] c_{1}, \quad c_{9}=\left[\frac{40 \lambda^{2}(1+\lambda)}{(-3+\lambda)(-2+\lambda)(-1+\lambda)(2+\lambda)}\right] c_{1}, \\
c_{8} & =\left[\frac{12 \lambda^{2}(3+\lambda)}{(-2+\lambda)(-1+\lambda)^{2}(2+\lambda)}\right] c_{1}, \quad c_{12}=0, \\
c_{10} & =-\left[\frac{2 \lambda^{2}(1+\lambda)\left(6+\lambda^{2}\right)}{N(-3+\lambda)(-2+\lambda)(-1+\lambda)(2+\lambda)}\right] c_{1}, \quad c_{11}=0, \quad\left[\frac{16 \lambda^{2}\left(-5-8 \lambda+\lambda^{2}\right)}{N(-3+\lambda)(-1+\lambda)^{2}(2+\lambda)}\right] c_{1},
\end{aligned}
$$

\footnotetext{
${ }^{15}$ Since the 'solve' command with 28 equations simultaneously in the mathematica does not give us the final answer, we consider several simple equations and find the coefficient functions in terms of $c_{1}$ and then solve the remaining linear equations completely.
} 


$$
\begin{aligned}
& c_{15}=\left[\frac{4 \lambda^{2}\left(3+\lambda^{2}\right)}{N^{2}(-3+\lambda)(-1+\lambda)^{2}}\right] c_{1}, \quad c_{17}=-\left[\frac{4 N^{2} \lambda}{3(2+\lambda)}\right] c_{1}, \\
& c_{20}=\left[\frac{4 N^{2} \lambda(1+\lambda)}{(-1+\lambda)(2+\lambda)}\right] c_{1}, \quad c_{22}=-\left[\frac{4 N^{2} \lambda(1+\lambda)}{3(-1+\lambda)(2+\lambda)}\right] c_{1} .
\end{aligned}
$$

Note that there are the $\frac{1}{N}$ dependence in $c_{5}, c_{10}, c_{13}, c_{14}$ and $\frac{1}{N^{2}}$ dependence in $c_{15}$ and $N^{2}$ dependence in $c_{17}, c_{20}, c_{22} 16$. Therefore, in the large $N$ limit, the only 11 independent terms in (3.20) survive.

\section{Appendix G The independent terms of $\widetilde{W}(z)$ in subsection 3.2}

Let us check how many independent terms in the coset spin 4 current $\widetilde{W}(z)$ in (3.16) occur. It is convenient to write down the terms of $c_{2}, c_{3}, c_{4}$ in the equation (3.16) explicitly from the relation (2.12)

$$
\begin{aligned}
& d^{a b c d} J^{a} J^{b} J^{c} K^{d}(z)=3 d^{a b c} J^{a} Q^{b} K^{c}(z)-\frac{12\left(N^{2}-4\right)}{N\left(N^{2}+1\right)} J^{a} J^{b} J^{b} K^{a}(z) \\
& +\frac{2\left(N^{2}-4\right)\left(N^{2}-3\right)}{N\left(N^{2}+1\right)} f^{a b c} \partial J^{a} J^{b} K^{c}(z)-\frac{\left(N^{2}-4\right)\left(N^{2}-3\right)}{N\left(N^{2}+1\right)} f^{a b c} J^{a} \partial J^{b} K^{c}(z), \\
& d^{a b c d} J^{a} J^{b} K^{c} K^{d}(z)=2 d^{a c e} d^{b d e} J^{a} J^{b} K^{c} K^{d}(z)+d^{a b c} J^{a} J^{b} R^{c}(z) \\
& -\frac{\left(N^{2}-4\right)\left(N^{2}-3\right)}{N\left(N^{2}+1\right)} f^{a b c} J^{a} J^{b} \partial K^{c}(z)-\frac{4\left(N^{2}-4\right)}{N\left(N^{2}+1\right)}\left[2 J^{a} J^{b} K^{a} K^{b}(z)+J^{a} J^{a} K^{b} K^{b}(z)\right] \\
& d^{a b c d} J^{a} K^{b} K^{c} K^{d}(z)=3 d^{a b c} J^{a} K^{b} R^{c}(z)-\frac{12\left(N^{2}-4\right)}{N\left(N^{2}+1\right)} J^{a} K^{a} K^{b} K^{b}(z) \\
& +\frac{2\left(N^{2}-4\right)\left(N^{2}-3\right)}{N\left(N^{2}+1\right)} f^{a b c} J^{a} \partial K^{b} K^{c}(z)-\frac{\left(N^{2}-4\right)\left(N^{2}-3\right)}{N\left(N^{2}+1\right)} f^{a b c} J^{a} K^{b} \partial K^{c}(z) .
\end{aligned}
$$

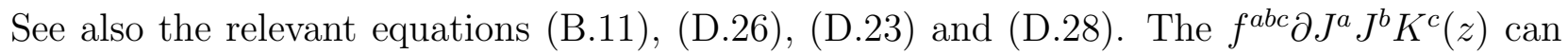
be written as a linear combination of $f^{a b c} J^{a} \partial J^{b} K^{c}(z)$ and $\partial^{2} J^{a} K^{a}(z)$. For given independent terms $c_{1}-c_{22}$ in (3.16), one regards $d^{a b c d} J^{a} J^{b} J^{c} K^{d}(z)$ as $f^{a b c} J^{a} \partial J^{b} K^{c}(z)$ and other terms, $d^{a b c d} J^{a} J^{b} K^{c} K^{d}(z)$ as both $d^{a c e} d^{b d e} J^{a} J^{b} K^{c} K^{d}(z)$ and $J^{a} J^{b} K^{a} K^{b}(z)$ (and other terms), and $d^{a b c d} J^{a} K^{b} K^{c} K^{d}(z)$ as $f^{a b c} J^{a} K^{b} \partial K^{c}(z)$ (and other terms). That is,

$$
\begin{aligned}
d^{a b c d} J^{a} J^{b} J^{c} K^{d}(z) & \sim f^{a b c} J^{a} \partial J^{b} K^{c}(z)+\cdots \\
d^{a b c d} J^{a} J^{b} K^{c} K^{d}(z) & \sim d^{a c e} d^{b d e} J^{a} J^{b} K^{c} K^{d}(z), J^{a} J^{b} K^{a} K^{b}(z)+\cdots \\
d^{a b c d} J^{a} K^{b} K^{c} K^{d}(z) & \sim f^{a b c} J^{a} K^{b} \partial K^{c}(z)+\cdots
\end{aligned}
$$

\footnotetext{
${ }^{16}$ Here we substitute $N=\frac{\lambda}{1-\lambda} k$ with $k_{1}=1, k_{2}=k$ into the relations (F.8), look at the power of $k$ in the numerator and denominator of the coefficient functions and take $k \rightarrow \infty$ limit. By reading off the power of $k$ and resubstituting $k=\frac{1-\lambda}{\lambda} N$ into the leading term, one gets the final results (F.10).
} 
This implies that one may further generalize the $c_{3}$ term by introducing two independent terms with two arbitrary coefficients. The $c_{1}$ term can be rewritten as the equation (3.17) and $c_{5}$ term can be expressed similarly by changing $J^{a}(z)$ into $K^{a}(z)$ from (3.17). We will use the explicit relations (G.1) to find the exact relation between the spin- 4 current (3.39) and the one from [5] where we do not see any quartic Casimir operator because in this case, the spin-4 current is obtained from the operator product expansions of two Casimir operators of rank 3. Therefore, the relations (G.1) are very important to identify these two spin- 4 currents.

Moreover, the other contractions of $d$ symbol (2.12) with various spin 4 fields lead to

$$
\begin{aligned}
d^{\text {ace }} d^{\text {bde }} J^{a} J^{b} J^{c} J^{d}(z) & =d^{a b e} d^{c d e} J^{a} J^{b} J^{c} J^{d}(z)-\left(N^{2}-4\right)\left[\partial J^{a} \partial J^{a}(z)-\partial^{2} J^{a} J^{a}(z)\right], \\
d^{\text {ade }} d^{b c e} J^{a} J^{b} J^{c} J^{d}(z) & =d^{a b e} d^{c d e} J^{a} J^{b} J^{c} J^{d}(z)-2\left(N^{2}-4\right) \partial J^{a} \partial J^{a}(z)+\left(N^{2}-4\right) \partial^{2} J^{a} J^{a}(z), \\
d^{a c e} d^{b d e} J^{a} J^{b} J^{c} K^{d}(z) & =d^{a b e} d^{c d e} J^{a} J^{b} J^{c} K^{d}(z)+\frac{N^{2}-4}{N} f^{a b c} J^{a} \partial J^{b} K^{c}(z), \\
d^{\text {ade }} d^{b c e} J^{a} J^{b} J^{c} K^{d}(z) & =d^{a b e} d^{c d e} J^{a} J^{b} J^{c} K^{d}(z)+\frac{N^{2}-4}{N} f^{a b c}\left[J^{a} \partial J^{b} K^{c}(z)-\partial J^{a} J^{b} K^{c}(z)\right], \\
d^{\text {ade }} d^{b c e} J^{a} J^{b} K^{c} K^{d}(z) & =d^{a c e} d^{b d e} J^{a} J^{b} K^{c} K^{d}(z)-\left(N^{2}-4\right) \partial J^{a} \partial K^{a}(z), \\
d^{a c e} d^{b d e} J^{a} K^{b} K^{c} K^{d}(z) & =d^{a b e} d^{c d e} J^{a} K^{b} K^{c} K^{d}(z)+\frac{N^{2}-4}{N} f^{a b c} J^{a} \partial K^{b} K^{c}(z), \\
d^{\text {ade }} d^{b c e} J^{a} K^{b} K^{c} K^{d}(z) & =d^{a b e} d^{c d e} J^{a} K^{b} K^{c} K^{d}(z)+\frac{N^{2}-4}{N} f^{a b c}\left[J^{a} \partial K^{b} K^{c}(z)-J^{a} K^{b} \partial K^{c}(z)\right], \\
d^{\text {ace }} d^{b d e} K^{a} K^{b} K^{c} K^{d}(z) & =d^{a b e} d^{c d e} K^{a} K^{b} K^{c} K^{d}(z)-\left(N^{2}-4\right)\left[\partial K^{a} \partial K^{a}(z)-\partial^{2} K^{a} K^{a}(z)\right], \\
d^{\text {ade }} d^{b c e} K^{a} K^{b} K^{c} K^{d}(z) & =d^{a b e} d^{c d e} K^{a} K^{b} K^{c} K^{d}(z)-2\left(N^{2}-4\right) \partial K^{a} \partial K^{a}(z) \\
& +\left(N^{2}-4\right) \partial^{2} K^{a} K^{a}(z) .
\end{aligned}
$$

From the relations (3.16), (G.1) and (G.2), there is no additional independent term. The contractions with delta symbol in the relation (2.12) can be written as

$$
\begin{aligned}
J^{a} J^{b} J^{a} J^{b}(z) & =J^{a} J^{a} J^{b} J^{b}(z)+f^{a b c} J^{a} \partial J^{b} J^{c}(z), \\
J^{a} J^{b} J^{b} J^{a}(z) & =J^{a} J^{a} J^{b} J^{b}(z)+f^{a b c} J^{a} \partial J^{b} J^{c}(z)-f^{a b c} J^{a} J^{b} \partial J^{c}(z), \\
J^{a} J^{b} J^{a} K^{b}(z) & =J^{a} J^{a} J^{b} K^{b}(z)+f^{a b c} J^{a} \partial J^{b} K^{c}(z), \\
J^{a} J^{b} J^{b} K^{a}(z) & =J^{a} J^{a} J^{b} K^{b}(z)+f^{a b c} J^{a} \partial J^{b} K^{c}(z)-f^{a b c} \partial J^{a} J^{b} K^{c}(z), \\
J^{a} J^{b} K^{b} K^{a}(z) & =J^{a} J^{b} K^{a} K^{b}(z)-f^{a b c} J^{a} J^{b} \partial K^{c}(z), \\
J^{a} K^{b} K^{a} K^{b}(z) & =J^{a} K^{a} K^{b} K^{b}(z)+f^{a b c} J^{a} \partial K^{b} K^{c}(z), \\
J^{a} K^{b} K^{b} K^{a}(z) & =J^{a} K^{a} K^{b} K^{b}(z)+f^{a b c} J^{a} \partial K^{b} K^{c}(z)-f^{a b c} J^{a} K^{b} \partial K^{c}(z), \\
K^{a} K^{b} K^{a} K^{b}(z) & =K^{a} K^{a} K^{b} K^{b}(z)+f^{a b c} K^{a} \partial K^{b} K^{c}(z), \\
K^{a} K^{b} K^{b} K^{a}(z) & =K^{a} K^{a} K^{b} K^{b}(z)+f^{a b c} K^{a} \partial K^{b} K^{c}(z)-f^{a b c} K^{a} K^{b} \partial K^{c}(z) .
\end{aligned}
$$


Note that one sees that the field $J^{a} J^{b} K^{a} K^{b}(z)$ occurs in (G.4). We do not see any further independent terms. Therefore, if we impose two independent terms in $c_{3}$ term, then we should include the field $J^{a} J^{b} K^{a} K^{b}(z)$ in the candidate for the spin 4 field (3.16). This can be also seen from the normal ordered field product $(\widetilde{T} \widetilde{T})(z)$.

In order to check the independent fields in $\widetilde{T}^{2}(z)$, one should write down the following normal ordered products

$$
\begin{aligned}
\left(J^{a} K^{a}\right)\left(J^{b} K^{b}\right)(z) & =J^{a} K^{a} J^{b} K^{b}(z)+N \partial^{2} J^{a} K^{a}(z)-\frac{k_{1}}{2} \partial^{2} K^{a} K^{a}(z)+f^{a b c} J^{a} \partial K^{b} K^{c}(z) \\
& -\frac{k_{2}}{2} J^{a} \partial^{2} J^{a}(z)-f^{a b c} J^{a} \partial J^{b} K^{c}(z) \\
\left(J^{a} K^{a}\right)\left(K^{b} K^{b}\right)(z) & =J^{a} K^{a} K^{b} K^{b}(z)-\left(N+k_{2}\right) \partial^{2} J^{a} K^{a}(z), \\
\left(J^{a} J^{a}\right)\left(J^{b} K^{b}\right)(z) & =J^{a} J^{a} J^{b} K^{b}(z)-\left(2 N+k_{1}\right) \partial^{2} J^{a} K^{a}(z)+2 f^{a b c} J^{a} \partial J^{b} K^{c}(z), \\
\left(K^{a} K^{a}\right)\left(J^{b} K^{b}\right)(z) & =J^{b} K^{a} K^{a} K^{b}(z)-\left(2 N+k_{2}\right) J^{a} \partial^{2} K^{a}(z)+2 f^{a b c} J^{a} K^{b} \partial K^{c}(z) \\
\left(J^{a} K^{a}\right)\left(J^{b} J^{b}\right)(z) & =J^{a} K^{a} J^{b} J^{b}(z)-\left(N+k_{1}\right) J^{a} \partial^{2} K^{a}(z) .
\end{aligned}
$$

The spin 4 current can be written, by using the above relations (G.5), as

$$
\begin{aligned}
\widetilde{T}^{2}(z) & =\frac{k_{2}^{2}}{4\left(N+k_{1}\right)^{2}\left(N+k_{1}+k_{2}\right)^{2}}\left[-2\left(k_{1}+N\right) \partial^{2} J^{a} J^{a}(z)+J^{a} J^{a} J^{b} J^{b}(z)\right] \\
& +\frac{k_{1}^{2}}{4\left(N+k_{2}\right)^{2}\left(N+k_{1}+k_{2}\right)^{2}}\left[-2\left(k_{2}+N\right) \partial^{2} K^{a} K^{a}(z)+K^{a} K^{a} K^{b} K^{b}(z)\right] \\
& +\frac{k_{1} k_{2}}{2\left(N+k_{1}\right)\left(N+k_{2}\right)\left(N+k_{1}+k_{2}\right)^{2}} J^{a} J^{a} K^{b} K^{b}(z) \\
& +\frac{1}{\left(N+k_{1}+k_{2}\right)^{2}}\left[J^{a} K^{a} J^{b} K^{b}(z)+N \partial^{2} J^{a} K^{a}-\frac{k_{1}}{2} \partial^{2} K^{a} K^{a}(z)+f^{a b c} J^{a} \partial K^{b} K^{c}(z)\right. \\
& \left.-\frac{k_{2}}{2} J^{a} \partial^{2} J^{a}(z)-f^{a b c} J^{a} \partial J^{b} K^{c}(z)\right] \\
& -\frac{k_{1}}{2\left(N+k_{2}\right)\left(N+k_{1}+k_{2}\right)^{2}}\left[J^{a} K^{a} K^{b} K^{b}(z)-\left(N+k_{2}\right) \partial^{2} J^{a} K^{a}(z)\right. \\
& \left.+J^{b} K^{a} K^{a} K^{b}(z)-\left(2 N+k_{2}\right) J^{a} \partial^{2} K^{a}(z)+2 f^{a b c} J^{a} K^{b} \partial K^{c}(z)\right] \\
& -\frac{k_{2}}{2\left(N+k_{1}\right)\left(N+k_{1}+k_{2}\right)^{2}}\left[J^{a} K^{a} J^{b} J^{b}(z)-\left(N+k_{1}\right) J^{a} \partial^{2} K^{a}(z)\right. \\
& \left.+J^{a} J^{a} J^{b} K^{b}(z)-\left(2 N+k_{1}\right) \partial^{2} J^{a} K^{a}(z)+2 f^{a b c} J^{a} \partial J^{b} K^{c}(z)\right] .
\end{aligned}
$$

Therefore, one can think of $\widetilde{T}^{2}(z)$ as an independent term $J^{a} J^{b} K^{a} K^{b}(z)$ plus others:

$$
\widetilde{T}^{2}(z) \sim J^{a} J^{b} K^{a} K^{b}(z)+\cdots .
$$

The terms $f^{a b c} J^{a} \partial J^{b} K^{c}(z)$ and $f^{a b c} J^{a} K^{b} \partial K^{c}(z)$ can be understood as $c_{2}$ term and $c_{4}$ term respectively from the relations (G.2). Although we introduce the extra terms $d^{\text {ace }} d^{\text {bde }}$ multiplied 
by the fields appearing in $c_{6}-c_{10}$, the extra terms $d^{\text {ade }} d^{b c e}$ multiplied by the fields appearing in $c_{6}{ }^{-} c_{10}$, the extra terms $\delta^{a c} \delta^{b d}$ multiplied by the fields appearing in $c_{11^{-}} c_{15}$, and the extra terms $\delta^{a d} \delta^{b c}$ multiplied by the fields appearing in $c_{11}-c_{15}$, there exists only one further independent term which is given by $J^{a} J^{b} K^{a} K^{b}(z)$. We will use the explicit form of (G.6) in order to extract the spin-4 field next Appendix $I$ later and the large $N$ limit for (G.6) can be obtained.

Or one can add $\widetilde{T}^{2}(z)$ (or $\left.\widetilde{\Lambda}(z) \equiv \widetilde{T}^{2}(z)-\frac{3}{10} \partial^{2} \widetilde{T}(z)\right)$ to the $\widetilde{W}(z)(3.16)$ in order to see any further generalized spin 4 field. We can use the operator product expansion

$$
\widetilde{T}(z) \widetilde{\Lambda}(w)=\frac{1}{(z-w)^{4}}\left[\frac{22}{5}+\widetilde{c}\right] \widetilde{T}(w)+\frac{1}{(z-w)^{2}} 4 \widetilde{\Lambda}(w)+\frac{1}{(z-w)} \partial \widetilde{\Lambda}(w)+\cdots
$$

and $J^{\prime a}(z) \widetilde{\Lambda}(w)=$ regular and the central charge $\widetilde{c}$ is given by the equation (3.9). This particular combination eliminates the singular terms with $\frac{1}{(z-w)^{6}}$ and $\frac{1}{(z-w)^{3}}$ that appear in (C.8). The fields $\widetilde{T}^{2}(z)$ and $\widetilde{\Lambda}(z)$ are quasiprimary [10] because there are higher order singular terms (G.8) and (C.8) where $T(z)$ and $c$ are replaced with $\widetilde{T}(z)$ and $\widetilde{c}$ respectively. In the subsection 3.4, we include the field $J^{a} J^{b} K^{a} K^{b}(z)$ rather than $\widetilde{T}^{2}(z)$. Contrary to the spin-3 case, one should, in general, take into account of the normal ordered field product of stress energy tensor for higher spin greater than 3.

\section{Appendix $\mathrm{H}$ The coefficient functions in the subsection 3.4}

The operator product expansion between the diagonal spin 1 current $J^{\prime a}(z)$ (3.4) and the spin 4 field $J^{b} J^{c} K^{b} K^{c}(w)$ is given by

$$
\begin{aligned}
& J^{a}(z) J^{b} J^{c} K^{b} K^{c}(w)=\frac{1}{(z-w)^{3}}(-N)\left[k_{1} \partial K^{a}+k_{2} \partial J^{a}\right]+\frac{1}{(z-w)^{2}}\left[-\frac{4}{N} J^{a} K^{b} K^{b}\right. \\
& +\left(\frac{4}{N}-2 k_{1}\right) J^{b} K^{a} K^{b}-d^{a b c} J^{b} R^{c}-d^{a b c} Q^{b} K^{c}+d^{a c e} d^{b d e} J^{b} K^{c} K^{d}+d^{a c e} d^{b d e} J^{c} J^{d} K^{b} \\
& \left.-\left(N-k_{1}\right) f^{a b c} J^{b} \partial K^{c}+\left(N-k_{2}\right) f^{a b c} \partial J^{b} K^{c}-\frac{4}{N} J^{b} J^{b} K^{a}+\left(\frac{4}{N}-2 k_{2}\right) J^{a} J^{b} K^{b}\right] \\
& +\cdots
\end{aligned}
$$

By realizing the independent field contents described in (F.2), one can arrange each field in the $\frac{1}{(z-w)^{2}}$ term in the relation ( $(\mathbf{H . 1})$ and combine them with the linear equations (F.1), (F.3) and (F.5). The operator product expansion between the stress energy tensor (E.1) and $J^{b} J^{c} K^{b} K^{c}(z)$ is

$$
\hat{T}(z) J^{b} J^{c} K^{b} K^{c}(w)=-\frac{1}{(z-w)^{4}}\left[k_{2} J^{a} J^{a}+k_{1} K^{a} K^{a}\right]+\frac{1}{(z-w)^{3}} N \partial\left(J^{a} K^{a}\right)+\cdots,
$$


where the $\frac{1}{(z-w)^{2}}$ term and $\frac{1}{(z-w)}$ term are ignored. One should include this equation into (F.5) and (F.6).

As in the subsection [3.4, let us introduce the coefficient $c_{23}$ in front of the extra field $J^{b} J^{c} K^{b} K^{c}(z)$. Among 18 equations (F.1), the first four and the last four equations are unchanged and the rest should be modified because of the extra terms in $\frac{1}{(z-w)^{2}}$ terms of the operator product expansion ( $(\underline{H .1})$. The first two equations of (F.3) have to be modified due to the $\frac{1}{(z-w)^{3}}$ term in the operator product expansion (․․1). The first and fourth equations in (F.5) should be changed due to the $\frac{1}{(z-w)^{3}}$ in the operator product expansion (․․2). Finally, the first and third equations in ( $(\overline{\text { F.6 }})$ have to be changed from the $\frac{1}{(z-w)^{4}}$ terms in the operator product expansion ( $(\mathbf{H . 2})$. Then the coefficients $c_{i}$ should be modified as $\left(c_{i}+b_{i}\right)$, when we add the extra field $J^{a} J^{b} K^{a} K^{b}(z)$, where the coefficients $b_{i}$ depend on $c_{23}$ (there is no constraint on $c_{23}$ ) and they are given by

$$
\begin{aligned}
& b_{2}=\frac{\left(k_{2}^{2}-1\right) N^{2}\left(N^{2}+1\right)^{2}}{3\left(N^{2}-4\right)\left(N+2 k_{1}\right)\left(N^{2}-3\right) D\left(k_{1}, k_{2}, N\right)}\left[-6 k_{1}^{3}-6 k_{1}^{2} k_{2}-17 k_{1}^{2} N-5 k_{1} k_{2} N\right. \\
& \left.-12 k_{1} N^{2}+2 k_{1}^{3} N^{2}+2 k_{2} N^{2}+2 k_{1}^{2} k_{2} N^{2}+7 k_{1}^{2} N^{3}+3 k_{1} k_{2} N^{3}+6 k_{1} N^{4}\right] c_{23} \text {, } \\
& b_{3}=-\frac{N\left(N^{2}+1\right)}{4\left(N^{2}-4\right) D\left(k_{1}, k_{2}, N\right)}\left[12 k_{1}^{3}+24 k_{1}^{2} k_{2}+12 k_{1} k_{2}^{2}+44 k_{1}^{2} N+52 k_{1} k_{2} N+2 k_{1}^{3} k_{2} N\right. \\
& +8 k_{2}^{2} N+2 k_{1}^{2} k_{2}^{2} N+48 k_{1} N^{2}-8 k_{1}^{3} N^{2}+24 k_{2} N^{2}-9 k_{1}^{2} k_{2} N^{2}-k_{1} k_{2}^{2} N^{2}+16 N^{3} \\
& \text { - } 26 k_{1}^{2} N^{3}-20 k_{1} k_{2} N^{3}+2 k_{1}^{3} k_{2} N^{3}+2 k_{2}^{2} N^{3}+2 k_{1}^{2} k_{2}^{2} N^{3}-24 k_{1} N^{4}-4 k_{2} N^{4}+7 k_{1}^{2} k_{2} N^{4} \\
& \left.+3 k_{1} k_{2}^{2} N^{4}-6 N^{5}+6 k_{1} k_{2} N^{5}\right] c_{23} \text {, } \\
& b_{4}=-\frac{N^{2}\left(N^{2}+1\right)^{2}}{6\left(N^{2}-4\right)\left(2 N+3 k_{2}\right)\left(N^{2}-3\right) D\left(k_{1}, k_{2}, N\right)}\left[-18 k_{1}^{2} k_{2}+18 k_{1}^{4} k_{2}-18 k_{1} k_{2}^{2}+18 k_{1}^{3} k_{2}^{2}\right. \\
& +12 k_{1}^{4} N-60 k_{1} k_{2} N+75 k_{1}^{3} k_{2} N-12 k_{2}^{2} N+12 k_{1}^{2} k_{2}^{2} N-15 k_{1} k_{2}^{3} N-32 k_{1} N^{2}+42 k_{1}^{3} N^{2} \\
& \text { - } 32 k_{2} N^{2}+84 k_{1}^{2} k_{2} N^{2}-6 k_{1}^{4} k_{2} N^{2}-40 k_{1} k_{2}^{2} N^{2}-6 k_{1}^{3} k_{2}^{2} N^{2}-10 k_{2}^{3} N^{2}-16 N^{3}+52 k_{1}^{2} N^{3} \\
& +16 k_{1} k_{2} N^{3}-21 k_{1}^{3} k_{2} N^{3}-32 k_{2}^{2} N^{3}-4 k_{1}^{2} k_{2}^{2} N^{3}+5 k_{1} k_{2}^{3} N^{3}+26 k_{1} N^{4}-18 k_{2} N^{4} \\
& \text { - } \left.18 k_{1}^{2} k_{2} N^{4}+10 k_{1} k_{2}^{2} N^{4}-12 k_{1}^{2} N\right] c_{23} \text {, } \\
& b_{5}=-\frac{k_{1}\left(k_{1}-k_{2}\right) N^{2}\left(3 N+2 k_{1}+2 k_{2}\right)\left(N^{2}+1\right)^{2}}{8\left(N^{2}-4\right)\left(2 N+3 k_{2}\right)\left(N+k_{2}\right)\left(N^{2}-3\right) D\left(k_{1}, k_{2}, N\right)}\left[-3 k_{1}^{2} k_{2}-3 k_{1} k_{2}^{2}\right. \\
& \text { - } \left.2 k_{1}^{2} N-8 k_{1} k_{2} N-2 k_{2}^{2} N-4 k_{1} N^{2}-4 k_{2} N^{2}+k_{1}^{2} k_{2} N^{2}+k_{1} k_{2}^{2} N^{2}-2 N^{3}+2 k_{1} k_{2} N^{3}\right] c_{23} \text {, } \\
& b_{6}=-\frac{\left(k_{2}^{2}-1\right) k_{2} N^{2}\left(N^{2}+1\right)}{2\left(N^{2}-4\right)\left(N+2 k_{1}\right) D\left(k_{1}, k_{2}, N\right)}\left[k_{1}^{2}+k_{1} k_{2}+2 k_{1} N+2 k_{2} N+2 N^{2}+k_{1}^{2} N^{2}\right. \\
& \left.+k_{1} k_{2} N^{2}+2 k_{1} N^{3}\right] c_{23} \text {, } \\
& b_{7}=-\frac{\left(k_{2}^{2}-1\right) N^{3}\left(N^{2}+1\right)}{\left(N^{2}-4\right)\left(N+2 k_{1}\right)\left(N^{2}-3\right)}\left[k_{1}^{2}+13 k_{1} k_{2}+12 k_{1} N+14 k_{2} N+12 N^{2}+2 k_{1}^{2} N^{2}\right.
\end{aligned}
$$




$$
\begin{aligned}
& \left.-2 k_{1} k_{2} N^{2}-2 k_{1} N^{3}-2 k_{2} N^{3}-4 N^{4}+k_{1}^{2} N^{4}+k_{1} k_{2} N^{4}+2 k_{1} N^{5}\right] c_{23}, \\
& b_{8}=\frac{N\left(N^{2}+1\right)}{2\left(N^{2}-4\right)\left(N+2 k_{1}\right)\left(N+2 k_{2}\right) D\left(k_{1}, k_{2}, N\right)}\left[72 k_{1}^{4} k_{2}+144 k_{1}^{3} k_{2}^{2}+72 k_{1}^{2} k_{2}^{3}+36 k_{1}^{4} N\right. \\
& +360 k_{1}^{3} k_{2} N+408 k_{1}^{2} k_{2}^{2} N+84 k_{1} k_{2}^{3} N+144 k_{1}^{3} N^{2}+592 k_{1}^{2} k_{2} N^{2}-44 k_{1}^{4} k_{2} N^{2}+80 k_{2}^{2} N^{3} \\
& +328 k_{1} k_{2}^{2} N^{2}-88 k_{1}^{3} k_{2}^{2} N^{2}+24 k_{2}^{3} N^{2}-44 k_{1}^{2} k_{2}^{3} N^{2}+212 k_{1}^{2} N^{3}-4 k_{1}^{4} N^{3}+388 k_{1} k_{2} N^{3} \\
& \text { - } 184 k_{1}^{3} k_{2} N^{3}-219 k_{1}^{2} k_{2}^{2} N^{3}-39 k_{1} k_{2}^{3} N^{3}+136 k_{1} N^{4}-16 k_{1}^{3} N^{4}+88 k_{2} N^{4}-251 k_{1}^{2} k_{2} N^{4} \\
& +4 k_{1}^{4} k_{2} N^{4}-137 k_{1} k_{2}^{2} N^{4}+8 k_{1}^{3} k_{2}^{2} N^{4}-10 k_{2}^{3} N^{4}+4 k_{1}^{2} k_{2}^{3} N^{4}+32 N^{5}-26 k_{1}^{2} N^{5} \\
& -128 k_{1} k_{2} N^{5}+16 k_{1}^{3} k_{2} N^{5}-26 k_{2}^{2} N^{5}+17 k_{1}^{2} k_{2}^{2} N^{5}+k_{1} k_{2}^{3} N^{5}-20 k_{1} N^{6}-22 k_{2} N^{6} \\
& \left.+19 k_{1}^{2} k_{2} N^{6}+5 k_{1} k_{2}^{2} N^{6}-6 N^{7}+6 k_{1} k_{2} N^{7}\right] c_{23}, \\
& b_{9}=\frac{N^{3}\left(N^{2}+1\right)}{\left(N^{2}-4\right)\left(N+2 k_{2}\right)\left(2 N+3 k_{2}\right)\left(N^{2}-3\right) D\left(k_{1}, k_{2}, N\right)}\left[33 k_{1}^{2} k_{2}+18 k_{1}^{4} k_{2}-3 k_{1} k_{2}^{2}+18 k_{1}^{3} k_{2}^{2}\right. \\
& -51 k_{1}^{2} k_{2}^{3}-15 k_{1} k_{2}^{4}+30 k_{1}^{2} N+36 k_{1}^{4} N+46 k_{1} k_{2} N+99 k_{1}^{3} k_{2} N-2 k_{2}^{2} N-54 k_{1}^{2} k_{2}^{2} N \\
& -10 k_{2}^{4} N+44 k_{1} N^{2}+126 k_{1}^{3} N^{2}+16 k_{2} N^{2}+76 k_{1}^{2} k_{2} N^{2}+16 k_{1}^{4} k_{2} N^{2}-212 k_{1} k_{2}^{2} N^{2} \\
& +16 k_{1}^{3} k_{2}^{2} N^{2}-74 k_{2}^{3} N^{2}+2 k_{1}^{2} k_{2}^{3} N^{2}-10 k_{1} k_{2}^{4} N^{2}+16 N^{3}+116 k_{1}^{2} N^{3}-4 k_{1}^{4} N^{3}-82 k_{1} k_{2} N^{3} \\
& +52 k_{1}^{3} k_{2} N^{3}-114 k_{2}^{2} N^{3}+44 k_{1}^{2} k_{2}^{2} N^{3}-18 k_{1} k_{2}^{3} N^{3}-10 k_{2}^{4} N^{3}+10 k_{1} N^{4}-14 k_{1}^{3} N^{4}-66 k_{2} N^{4} \\
& +69 k_{1}^{2} k_{2} N^{4}-2 k_{1}^{4} k_{2} N^{4}+25 k_{1} k_{2}^{2} N^{4}-2 k_{1}^{3} k_{2}^{2} N^{4}-30 k_{2}^{3} N^{4}+5 k_{1}^{2} k_{2}^{3} N^{4}+5 k_{1} k_{2}^{4} N^{4} \\
& -16 N^{5}-10 k_{1}^{2} N^{5}+40 k_{1} k_{2} N^{5}-7 k_{1}^{3} k_{2} N^{5}-24 k_{2}^{2} N^{5}+2 k_{1}^{2} k_{2}^{2} N^{5}+15 k_{1} k_{2}^{3} N^{5} \\
& \left.+2 k_{1} N^{6}-6 k_{2} N^{6}-6 k_{1}^{2} k_{2} N^{6}+10 k_{1} k_{2}^{2} N^{6}-145 k_{1} k_{2}^{3} N\right] c_{23} \text {, } \\
& b_{10}=\frac{k_{1} N^{2}\left(N^{2}+1\right)}{4\left(N^{2}-4\right)\left(N+2 k_{2}\right)\left(N+k_{2}\right)\left(2 N+3 k_{2}\right)\left(N^{2}-3\right) D\left(k_{1}, k_{2}, N\right)}\left[-18 k_{1}^{2} k_{2}^{2}-18 k_{1} k_{2}^{3}\right. \\
& +18 k_{1} k_{2}^{5}-54 k_{1}^{2} k_{2} N-18 k_{1}^{4} k_{2} N-66 k_{1} k_{2}^{2} N-18 k_{1}^{3} k_{2}^{2} N-12 k_{2}^{3} N+72 k_{1}^{2} k_{2}^{3} N \\
& +84 k_{1} k_{2}^{4} N+12 k_{2}^{5} N-36 k_{1}^{2} N^{2}-36 k_{1}^{4} N^{2}-108 k_{1} k_{2} N^{2}-99 k_{1}^{3} k_{2} N^{2}-36 k_{2}^{2} N^{2}+48 k_{1}^{2} k_{2}^{2} N^{2} \\
& +195 k_{1} k_{2}^{3} N^{2}+48 k_{2}^{4} N^{2}+12 k_{1}^{2} k_{2}^{4} N^{2}+12 k_{1} k_{2}^{5} N^{2}-60 k_{1} N^{3}-126 k_{1}^{3} N^{2}-48 k_{2} N^{3} \\
& \text { - } 90 k_{1}^{2} k_{2} N^{3}-16 k_{1}^{4} k_{2} N^{3}+178 k_{1} k_{2}^{2} N^{3}-16 k_{1}^{3} k_{2}^{2} N^{3}+94 k_{2}^{3} N^{3}+12 k_{1}^{2} k_{2}^{3} N^{3}+60 k_{1} k_{2}^{4} N^{3} \\
& +12 k_{2}^{5} N^{3}-24 N^{4}-120 k_{1}^{2} N^{4}+4 k_{1}^{4} N^{4}+36 k_{1} k_{2} N^{4}-52 k_{1}^{3} k_{2} N^{4}+84 k_{2}^{2} N^{4}-34 k_{1}^{2} k_{2}^{2} N^{4} \\
& +70 k_{1} k_{2}^{3} N^{4}+48 k_{2}^{4} N^{4}-6 k_{1}^{2} k_{2}^{4} N^{4}-6 k_{1} k_{2}^{5} N^{4}-22 k_{1} N^{5}+14 k_{1}^{3} N^{5}+34 k_{2} N^{5} \\
& \text { - } 62 k_{1}^{2} k_{2} N^{5}+2 k_{1}^{4} k_{2} N^{5}+6 k_{1} k_{2}^{2} N^{5}+2 k_{1}^{3} k_{2}^{2} N^{5}+62 k_{2}^{3} N^{5}-12 k_{1}^{2} k_{2}^{3} N^{5}-24 k_{1} k_{2}^{4} N^{5} \\
& +8 N^{6}+12 k_{1}^{2} N^{6}-24 k_{1} k_{2} N^{6}+7 k_{1}^{3} k_{2} N^{6}+32 k_{2}^{2} N^{6}-4 k_{1}^{2} k_{2}^{2} N^{6}-31 k_{1} k_{2}^{3} N^{6}+2 k_{1} N^{7} \\
& \left.+6 k_{2} N^{7}+6 k_{1}^{2} k_{2} N^{7}-14 k_{1} k_{2}^{2} N^{7}+18 k_{1}^{2} k_{2}^{4}\right] c_{23} \text {, } \\
& b_{11}=\frac{\left(k_{2}^{2}-1\right) k_{2} N\left(N^{2}+1\right)\left(3 N+2 k_{1}+2 k_{2}\right)}{2 D\left(k_{1}, k_{2}, N\right)} c_{23} \text {, }
\end{aligned}
$$




$$
\begin{aligned}
& b_{12}=\frac{2\left(k_{2}^{2}-1\right) N^{2}\left(N^{2}+1\right)}{\left(N+2 k_{1}\right)\left(N^{2}-3\right) D\left(k_{1}, k_{2}, N\right)}\left[2 k_{1}^{2}+8 k_{1} k_{2}+9 k_{1} N+10 k_{2} N\right. \\
& \left.+9 N^{2}+2 k_{1}^{2} N^{2}+k_{1} N^{3}-2 k_{2} N^{3}-3 N^{4}\right] c_{23}, \\
& b_{13}=\frac{1}{D\left(k_{1}, k_{2}, N\right)}\left[-18 k_{1}^{3}-36 k_{1}^{2} k_{2}-18 k_{1} k_{2}^{2}-63 k_{1}^{2} N-75 k_{1} k_{2} N-12 k_{2}^{2} N-67 k_{1} N^{2}\right. \\
& +2 k_{1}^{3} N^{2}-34 k_{2} N^{2}+4 k_{1}^{2} k_{2} N^{2}-k_{1} k_{2}^{2} N^{2}-22 N^{3}+7 k_{1}^{2} N^{3} \\
& \left.+3 k_{1} k_{2} N^{3}-6 k_{2}^{2} N^{3}+5 k_{1} N^{4}-6 k_{2} N^{4}+k_{1} k_{2}^{2} N^{4}\right] c_{23}, \\
& b_{14}=-\frac{2 N^{2}\left(N^{2}+1\right)}{\left(2 N+3 k_{2}\right)\left(N^{2}-3\right) D\left(k_{1}, k_{2}, N\right)}\left[6 k_{1}^{2}+18 k_{1}^{4}-3 k_{1} k_{2}+18 k_{1}^{3} k_{2}-24 k_{1}^{2} k_{2}^{2}-15 k_{1} k_{2}^{3}\right. \\
& +7 k_{1} N+63 k_{1}^{3} N-2 k_{2} N+12 k_{1}^{2} k_{2} N-70 k_{1} k_{2}^{2} N-10 k_{2}^{3} N+2 N^{2}+64 k_{1}^{2} N^{2} \\
& \text { - } 2 k_{1}^{4} N^{2}-39 k_{1} k_{2} N^{2}-2 k_{1}^{3} k_{2} N^{2}-36 k_{2}^{2} N^{2}+8 k_{1}^{2} k_{2}^{2} N^{2}+5 k_{1} k_{2}^{3} N^{2}+13 k_{1} N^{3} \\
& \text { - } \left.7 k_{1}^{3} N^{3}-28 k_{2} N^{3}+4 k_{1}^{2} k_{2} N^{3}+18 k_{1} k_{2}^{2} N^{3}-6 N^{4}-6 k_{1}^{2} N^{4}+12 k_{1} k_{2} N^{4}\right] c_{23}, \\
& b_{15}=-\frac{N k_{1}\left(3 N+2 k_{1}+2 k_{2}\right)\left(N^{2}+1\right)}{2\left(N+k_{2}\right)\left(2 N+3 k_{2}\right)\left(N^{2}-3\right) D\left(k_{1}, k_{2}, N\right)}\left[-9 k_{1} k_{2}+9 k_{1} k_{2}^{3}-9 k_{1} N-9 k_{1}^{3} N\right. \\
& \text { - } 6 k_{2} N+18 k_{1} k_{2}^{2} N+6 k_{2}^{3} N-6 N^{2}-18 k_{1}^{2} N^{2}+15 k_{1} k_{2} N^{2}+12 k_{2}^{2} N^{2}-3 k_{1} k_{2}^{3} N^{2} \\
& \left.-3 k_{1} N^{3}+k_{1}^{3} N^{3}+8 k_{2} N^{3}-6 k_{1} k_{2}^{2} N^{3}+2 N^{4}+2 k_{1}^{2} N^{4}-4 k_{1} k_{2} N^{4}\right] c_{23}, \\
& b_{16}=0 \\
& b_{17}=-\frac{\left(k_{2}^{2}-1\right) N^{2}\left(N^{2}+1\right)}{6\left(N+2 k_{1}\right) D\left(k_{1}, k_{2}, N\right)}\left[-6 k_{1}^{3}-6 k_{1}^{2} k_{2}-17 k_{1}^{2} N-5 k_{1} k_{2} N\right. \\
& \left.-12 k_{1} N^{2}+2 k_{1}^{3} N^{2}+2 k_{2} N^{2}+2 k_{1}^{2} k_{2} N^{2}+7 k_{1}^{2} N^{3}+3 k_{1} k_{2} N^{3}+6 k_{1} N^{4}\right] c_{23}, \\
& b_{18}=0 \\
& b_{19}=0 \text {, } \\
& b_{20}=\frac{N}{2 D\left(k_{1}, k_{2}, N\right)}\left[18 k_{1}^{3}+36 k_{1}^{2} k_{2}+18 k_{1} k_{2}^{2}+66 k_{1}^{2} N+78 k_{1} k_{2} N+12 k_{2}^{2} N-3 k_{1}^{2} k_{2}^{2} N\right. \\
& \text { - } 3 k_{1} k_{2}^{3} N+72 k_{1} N^{2}-20 k_{1}^{3} N^{2}+36 k_{2} N^{2}-40 k_{1}^{2} k_{2} N^{2}-22 k_{1} k_{2}^{2} N^{2}-2 k_{2}^{3} N^{2} \\
& +24 N^{3}-68 k_{1}^{2} N^{3}-76 k_{1} k_{2} N^{3}-8 k_{2}^{2} N^{3}-2 k_{1}^{2} k_{2}^{2} N^{3}-2 k_{1} k_{2}^{3} N^{3}-68 k_{1} N^{4} \\
& +2 k_{1}^{3} N^{4}-26 k_{2} N^{4}+4 k_{1}^{2} k_{2} N^{4}-6 k_{1} k_{2}^{2} N^{4}-2 k_{2}^{3} N^{4}-20 N^{5}+6 k_{1}^{2} N^{5}+2 k_{1} k_{2} N^{5} \\
& \text { - } \left.8 k_{2}^{2} N^{5}+k_{1}^{2} k_{2}^{2} N^{5}+k_{1} k_{2}^{3} N^{5}+4 k_{1} N^{6}-6 k_{2} N^{6}+2 k_{1} k_{2}^{2} N^{6}\right] c_{23}, \\
& b_{21}=0 \text {, } \\
& b_{22}=-\frac{\left(k_{2}^{2}-1\right) N^{2}\left(2 N+k_{1}+k_{2}\right)\left(N^{2}+1\right)\left(-3 k_{1}-2 N+k_{1} N^{2}\right)}{6 D\left(k_{1}, k_{2}, N\right)} c_{23} .
\end{aligned}
$$

Since the second and third equations in (F.5) and the equation (F.7) remain unchanged, one sees that the coefficients $b_{16}, b_{18}, b_{19}$ and $b_{21}$ should vanish. The $D\left(k_{1}, k_{2}, N\right)$ is defined as 
(F.9). Furthermore, the large $N$ limit (3.19) on these coefficients leads to

$$
\begin{aligned}
& b_{2}=-\left[\frac{(-1+\lambda)(1+\lambda)}{2 \lambda(2+\lambda)}\right] c_{23}, \quad b_{3}=-\left[\frac{3(1+\lambda)}{8(2+\lambda)}\right] c_{23}, \\
& b_{4}=-\left[\frac{5(-1+\lambda)(1+\lambda)}{12(-3+\lambda)(2+\lambda)}\right] c_{23}, \quad b_{5}=\left[\frac{(-1+\lambda)(1+\lambda)}{16 N(-3+\lambda)}\right] c_{23}, \\
& b_{6}=-\left[\frac{(-1+\lambda)^{2}(1+\lambda)}{4 \lambda^{2}(2+\lambda)}\right] c_{23}, \quad b_{7}=\left[\frac{(-1+\lambda)(1+\lambda)}{2 \lambda(2+\lambda)}\right] c_{23}, \\
& b_{8}=-\left[\frac{(1+\lambda)(1+2 \lambda)}{4(-2+\lambda)(2+\lambda)}\right] c_{23}, \quad b_{9}=-\left[\frac{5(-1+\lambda)(1+\lambda)}{2(-3+\lambda)(-2+\lambda)(2+\lambda)}\right] c_{23}, \\
& b_{10}=\left[\frac{(-1+\lambda)(1+\lambda)\left(6+\lambda^{2}\right)}{8 N(-3+\lambda)(-2+\lambda)(2+\lambda)}\right] c_{23}, \quad b_{11}=\left[\frac{(-1+\lambda)^{2}}{4 \lambda^{2}}\right] c_{23}, \\
& b_{12}=\left[\frac{(-1+\lambda)}{\lambda}\right] c_{23}, \quad b_{13}=-\left[\frac{(-1+\lambda)}{2 N(2+\lambda)}\right] c_{23}, \\
& b_{14}=-\left[\frac{\left(-5-8 \lambda+\lambda^{2}\right)}{N(-3+\lambda)(2+\lambda)}\right] c_{23}, \quad b_{15}=-\left[\frac{\left(3+\lambda^{2}\right)}{4 N^{2}(-3+\lambda)}\right] c_{23}, \\
& b_{17}=\left[\frac{N^{2}(-1+\lambda)(1+\lambda)}{4 \lambda(2+\lambda)}\right] c_{23}, \quad b_{20}=-\left[\frac{N^{2}(-1+\lambda)(1+\lambda)}{4 \lambda(2+\lambda)}\right] c_{23}, \\
& b_{22}=\left[\frac{N^{2}(-1+\lambda)(1+\lambda)}{12 \lambda(2+\lambda)}\right] c_{23} \text {. }
\end{aligned}
$$

There are the $\frac{1}{N}$ dependence in $b_{5}, b_{10}, b_{13}, b_{14}$ and $\frac{1}{N^{2}}$ dependence in $b_{15}$ and $N^{2}$ dependence in $b_{17}, b_{20}, b_{22}$. Then, the more general spin 4 field, in the large $N$ limit, can be written as (3.40). The extra terms proportional to $c_{23}$ in (3.40) appear also in (3.20). The contributions from $J^{a} J^{a} J^{b} J^{b}(z), J^{a} J^{a} J^{b} K^{b}(z)$ and $J^{a} J^{b} K^{a} K^{b}(z)$ in the large $N$ limit become zero.

\section{Appendix I The coset spin 4 primary field from the op- erator product expansion between the third- order Casimirs}

The primary field of dimension 4 under the stress energy tensor (3.6) and (3.7) has been constructed in [5] from the operator product expansion between the primary fields of dimension 3. That is, the coset spin 3 primary field is given by

$$
\widetilde{T}^{(3)}(z)=B d^{a b c}\left[A_{1} J^{a} J^{b} J^{c}(z)+A_{2} J^{a} J^{b} K^{c}(z)+A_{3} J^{a} K^{b} K^{c}(z)+A_{4} K^{a} K^{b} K^{c}(z)\right],
$$

where the coefficient functions that depend on $k_{1}, k_{2}$ or $N$ are determined by [5]

$$
B^{2}=-\frac{N}{18\left(N+k_{1}\right)^{2}\left(N+k_{2}\right)^{2}\left(N+k_{1}+k_{2}\right)^{2}\left(N+2 k_{1}\right)\left(N+2 k_{2}\right)\left(3 N+2 k_{1}+2 k_{2}\right)\left(N^{2}-4\right)},
$$




$$
\begin{aligned}
& A_{1}=k_{2}\left(N+k_{2}\right)\left(N+2 k_{2}\right), \\
& A_{2}=-3\left(N+k_{1}\right)\left(N+k_{2}\right)\left(N+2 k_{2}\right), \\
& A_{3}=3\left(N+k_{1}\right)\left(N+k_{2}\right)\left(N+2 k_{1}\right), \\
& A_{4}=-k_{1}\left(N+k_{1}\right)\left(N+2 k_{1}\right) .
\end{aligned}
$$

By focusing on the $\frac{1}{(z-w)^{2}}$ term in the operator product expansion between $\widetilde{T}^{(3)}(z)$ and $\widetilde{T}^{(3)}(w)$ which can be constructed from the relation (I.1), one obtains

$$
\begin{aligned}
& \widetilde{R}^{(4)}(w)+\frac{32}{22+5 \widetilde{c}} \widetilde{\Lambda}(w)+\frac{3}{10} \partial^{2} \widetilde{T}(w)= \\
& d^{a b c d}\left(d_{2} J^{a} J^{b} J^{c} K^{d}(w)+d_{3} J^{a} J^{b} K^{c} K^{d}(w)+d_{4} J^{a} K^{b} K^{c} K^{d}(w)\right) \\
& +d^{a b e} d^{c d e}\left(d_{6} J^{a} J^{b} J^{c} J^{d}(w)+d_{8} J^{a} J^{b} K^{c} K^{d}(w)+d_{10} K^{a} K^{b} K^{c} K^{d}(w)\right) \\
& +d_{12} J^{a} J^{a} J^{b} K^{b}(w)+d_{13} J^{a} J^{a} K^{b} K^{b}(w)+d_{14} J^{a} K^{a} K^{b} K^{b}(w)+d_{16} \partial^{2} J^{a} J^{a}(w) \\
& +d_{17} \partial^{2} J^{a} K^{a}(w)+d_{18} \partial^{2} K^{a} K^{a}(w)+d_{20} \partial J^{a} \partial K^{a}(w)+d_{22} J^{a} \partial^{2} K^{a}(w) \\
& +d_{23} J^{a} J^{b} K^{a} K^{b}(w)+d_{24} f^{a b c} J^{a} \partial J^{b} K^{c}(w)+d_{25} f^{a b c} J^{a} K^{b} \partial K^{c}(w) .
\end{aligned}
$$

Then a new spin-4 field $\widetilde{R}^{(4)}(w)$ can be read off from (1.3). While there are new terms characterized by $d_{23}, d_{24}$ and $d_{25}$, but there are missing terms corresponding to $c_{1}, c_{5}, c_{7}, c_{9}, c_{11}$, and $c_{15}$. Of course, it is not so simple to see the first three terms on the right hand side of (I.3) . Here the coefficient functions on the right hand side of (I.3) depend on $k_{1}, k_{2}$ or $N$ and they are determined by, together with the relation (I. $\mathbf{L}$ ),

$$
\begin{aligned}
d_{2} & =\left[-4 A_{1} A_{2}\left(N+k_{1}\right)-\frac{2}{3} A_{2} A_{3}\left(N+2 k_{2}\right)+\frac{2}{3} A_{2}^{2} N\right] B^{2} \\
& =12\left(N+k_{1}\right)^{2}\left(N+k_{2}\right)^{2}\left(N+k_{1}+k_{2}\right)\left(N+2 k_{2}\right)^{2} B^{2}, \\
d_{3} & =\left[-A_{2}^{2}\left(N+2 k_{1}\right)-A_{3}^{2}\left(N+2 k_{2}\right)\right] B^{2} \\
& =-18\left(N+k_{1}\right)^{2}\left(N+2 k_{1}\right)\left(N+k_{2}\right)^{2}\left(N+k_{1}+k_{2}\right)\left(N+2 k_{2}\right) B^{2}, \\
d_{4} & =\left[-\frac{2}{3} A_{2} A_{3}\left(N+2 k_{1}\right)-4 A_{3} A_{4}\left(N+k_{2}\right)+\frac{2}{3} A_{3}^{2} N\right] B^{2} \\
& =12\left(N+k_{1}\right)^{2}\left(N+2 k_{1}\right)^{2}\left(N+k_{2}\right)^{2}\left(N+k_{1}+k_{2}\right) B^{2}, \\
d_{6} & =\left[-9 A_{1}^{2}\left(N+k_{1}\right)-A_{2}^{2} k_{2}\right] B^{2}=-9 k_{2}\left(N+k_{1}\right)\left(N+k_{2}\right)^{2}\left(N+k_{1}+k_{2}\right)\left(N+2 k_{2}\right)^{2} B^{2}, \\
d_{8} & =\left[-6 A_{1} A_{3}\left(N+k_{1}\right)+4 A_{2} A_{3} N-6 A_{2} A_{4}\left(N+k_{2}\right)+2 A_{2}^{2} k_{1}+2 A_{3}^{2} k_{2}\right] B^{2} \\
& =-36 N\left(N+k_{1}\right)^{2}\left(N+k_{2}\right)^{2}\left(N+k_{1}+k_{2}\right)^{2} B^{2}, \\
d_{10} & =\left[-9 A_{4}^{2}\left(N+k_{2}\right)-A_{3}^{2} k_{1}\right] B^{2}=-9 k_{1}\left(N+k_{1}\right)^{2}\left(N+2 k_{1}\right)^{2}\left(N+k_{2}\right)\left(N+k_{1}+k_{2}\right) B^{2}, \\
d_{12} & =\left[-\frac{48\left(N^{2}-4\right)\left(N+k_{1}\right)}{N\left(N^{2}+1\right)} A_{1} A_{2}-\frac{8\left(N^{2}-4\right)\left(N+2 k_{2}\right)}{N\left(N^{2}+1\right)} A_{2} A_{3}+\frac{8\left(N^{2}-4\right)}{N^{2}+1} A_{2}^{2}\right] B^{2}
\end{aligned}
$$




$$
\begin{aligned}
& =\frac{144}{N\left(N^{2}+1\right)}\left(N^{2}-4\right)\left(N+k_{1}\right)^{2}\left(N+k_{2}\right)^{2}\left(N+k_{1}+k_{2}\right)\left(N+2 k_{2}\right)^{2} B^{2}, \\
& d_{13}=\left[-\frac{4\left(N^{2}-4\right)\left(N+2 k_{1}\right)}{N\left(N^{2}+1\right)} A_{2}^{2}-\frac{4\left(N^{2}-4\right)\left(N+2 k_{2}\right)}{N\left(N^{2}+1\right)} A_{3}^{2}\right] B^{2} \\
& =-\frac{72}{N\left(N^{2}+1\right)}\left(N^{2}-4\right)\left(N+k_{1}\right)^{2}\left(N+2 k_{1}\right)\left(N+k_{2}\right)^{2}\left(N+k_{1}+k_{2}\right)\left(N+2 k_{2}\right) B^{2}, \\
& d_{14}=\left[-\frac{8\left(N+2 k_{1}\right)\left(N^{2}-4\right)}{N\left(N^{2}+1\right)} A_{2} A_{3}-\frac{48\left(N+k_{2}\right)\left(N^{2}-4\right)}{N\left(N^{2}+1\right)} A_{3} A_{4}+\frac{8\left(N^{2}-4\right)}{N^{2}+1} A_{3}^{2}\right] B^{2} \\
& =\frac{144}{N\left(N^{2}+1\right)}\left(N^{2}-4\right)\left(N+k_{1}\right)^{2}\left(N+2 k_{1}\right)^{2}\left(N+k_{2}\right)^{2}\left(N+k_{1}+k_{2}\right) B^{2}, \\
& d_{16}=\left[\frac{9}{N} A_{1}^{2}\left(N+k_{1}\right)\left(N+2 k_{1}\right)\left(N^{2}-4\right)+\frac{2 k_{2}}{N}\left(N^{2}-4\right)\left(N+2 k_{1}\right) A_{2}^{2}\right. \\
& \left.+\frac{1}{N}\left(N^{2}-4\right) k_{2}\left(N+2 k_{2}\right) A_{3}^{2}\right] B^{2} \\
& =\frac{1}{N} 9 k_{2}\left(N^{2}-4\right)\left(N+k_{1}\right)\left(N+2 k_{1}\right)\left(N+k_{2}\right)^{2}\left(N+k_{1}+k_{2}\right)\left(N+2 k_{2}\right)\left(3 N+2 k_{1}+2 k_{2}\right) B^{2} \text {, } \\
& d_{17}=\left[\frac{8\left(N+k_{1}\right)\left(N^{2}-4\right)\left(N^{2}+3\right)}{N^{2}+1} A_{1} A_{2}+\frac{3}{N} A_{1} A_{2}\left(N+k_{1}\right)\left(N+2 k_{1}\right)\left(N^{2}-4\right)\right. \\
& +\frac{4\left(N+2 k_{2}\right)\left(N^{2}-4\right)\left(N^{2}+3\right)}{3\left(N^{2}+1\right)} A_{2} A_{3}+\frac{1}{N}\left(N+2 k_{1}\right)\left(N+2 k_{2}\right)\left(N^{2}-4\right) A_{2} A_{3} \\
& +\frac{3}{N}\left(N+k_{2}\right)\left(N+2 k_{2}\right)\left(N^{2}-4\right) A_{3} A_{4}+\left(-2\left(N+2 k_{1}\right)\left(N^{2}-4\right)\right. \\
& \left.\left.-\frac{4 N\left(N^{2}-4\right)\left(N^{2}+3\right)}{3\left(N^{2}+1\right)}\right) A_{2}^{2}-2 A_{3}^{2}\left(N+2 k_{2}\right)\left(N^{2}-4\right)\right] B^{2} \\
& =\frac{1}{N\left(N^{2}+1\right)} 3\left(N^{2}-4\right)\left(N+k_{1}\right)^{2}\left(N+k_{2}\right)^{2}\left(N+k_{1}+k_{2}\right)\left(N+2 k_{2}\right)\left[12 k_{1}^{2}+12 k_{1} k_{2}+36 k_{1} N\right. \\
& \left.+54 k_{2} N+39 N^{2}+12 k_{1} k_{2} N^{2}+36 k_{1} N^{3}+22 k_{2} N^{3}+23 N^{4}\right] B^{2}, \\
& d_{18}=\left[\frac{9}{N}\left(N+k_{2}\right)\left(N+2 k_{2}\right)\left(N^{2}-4\right) A_{4}^{2}+\frac{1}{N}\left(N^{2}-4\right) k_{1}\left(N+2 k_{1}\right) A_{2}^{2}\right. \\
& \left.+\frac{2 k_{1}}{N}\left(N^{2}-4\right)\left(N+2 k_{2}\right) A_{3}^{2}\right] B^{2} \\
& =\frac{9}{N} k_{1}\left(N^{2}-4\right)\left(N+k_{1}\right)^{2}\left(N+2 k_{1}\right)\left(N+k_{2}\right)\left(N+k_{1}+k_{2}\right)\left(N+2 k_{2}\right)\left(3 N+2 k_{1}+2 k_{2}\right) B^{2} \text {, } \\
& d_{20}=\left[-\frac{\left(N+2 k_{1}\right)\left(N^{2}-4\right)\left(N^{2}-3\right)}{N^{2}+1} A_{2}^{2}-\frac{\left(N+2 k_{2}\right)\left(N^{2}-4\right)\left(N^{2}-3\right)}{N^{2}+1} A_{3}^{2}\right] B^{2} \\
& =-\frac{18}{\left(N^{2}+1\right)}\left(N^{2}-4\right)\left(N+k_{1}\right)^{2}\left(N+2 k_{1}\right)\left(N+k_{2}^{2}\right)\left(N+k_{1}+k_{2}\right)\left(N+2 k_{2}\right)\left(N^{2}-3\right) B^{2}, \\
& d_{22}=\left[\frac{3}{N}\left(N+k_{1}\right)\left(N+2 k_{1}\right)\left(N^{2}-4\right) A_{1} A_{2}+\frac{4\left(N+2 k_{1}\right)\left(N^{2}-4\right)\left(N^{2}-3\right)}{3\left(N^{2}+1\right)} A_{2} A_{3}\right.
\end{aligned}
$$




$$
\begin{aligned}
& +\frac{1}{N}\left(N+2 k_{1}\right)\left(N+2 k_{2}\right)\left(N^{2}-4\right) A_{2} A_{3}+\frac{8\left(N^{2}-4\right)\left(N^{2}-3\right)\left(N+k_{2}\right)}{N^{2}+1} A_{3} A_{4} \\
& +\frac{3}{N}\left(N+2 k_{2}\right)\left(N^{2}-4\right)\left(N+k_{2}\right) A_{3} A_{4}-2\left(N+2 k_{1}\right)\left(N^{2}-4\right) A_{2}^{2}+\frac{8 N\left(N^{2}-4\right)}{N^{2}+1} A_{3}^{2} \\
& \left.+\left(-2\left(N+2 k_{2}\right)\left(N^{2}-4\right)-\frac{4 N\left(N^{2}-4\right)\left(N^{2}+3\right)}{3\left(N^{2}+1\right)}\right) A_{3}^{2}\right] B^{2} \\
& =-\frac{1}{N\left(N^{2}+1\right)} 3\left(N^{2}-4\right)\left(N+k_{1}\right)^{2}\left(N+2 k_{1}\right)\left(N+k_{2}\right)^{2}\left(N+k_{1}+k_{2}\right)\left[12 k_{1} k_{2}+12 k_{2}^{2}\right. \\
& \text { - } \left.42 k_{1} N+36 k_{2} N-9 N^{2}+12 k_{1} k_{2} N^{2}+12 k_{2}^{2} N^{2}+22 k_{1} N^{3}+36 k_{2} N^{3}+23 N^{4}\right] B^{2} \text {, } \\
& d_{23}=\left[-\frac{8\left(N^{2}-4\right)\left(N+2 k_{1}\right)}{N\left(N^{2}+1\right)} A_{2}^{2}-\frac{8\left(N^{2}-4\right)\left(N+2 k_{2}\right)}{N\left(N^{2}+1\right)} A_{3}^{2}\right] B^{2} \\
& =-\frac{144}{N\left(N^{2}+1\right)}\left(N^{2}-4\right)\left(N+k_{1}\right)^{2}\left(N+2 k_{1}\right)\left(N+k_{2}\right)^{2}\left(N+k_{1}+k_{2}\right)\left(N+2 k_{2}\right) B^{2} \text {, } \\
& d_{24}=\left[-\frac{12\left(N+k_{1}\right)\left(N^{2}-4\right)\left(N^{2}+5\right)}{N\left(N^{2}+1\right)} A_{1} A_{2}-\frac{2\left(N+2 k_{2}\right)\left(N^{2}-4\right)\left(N^{2}+5\right)}{N\left(N^{2}+1\right)} A_{2} A_{3}\right. \\
& \left.+\left(\frac{2}{N}\left(N+2 k_{1}\right)\left(N^{2}-4\right)+\frac{2\left(N^{2}-4\right)\left(N^{2}+5\right)}{N^{2}+1}\right) A_{2}^{2}+\frac{2}{N}\left(N^{2}-4\right)\left(N+2 k_{2}\right) A_{3}^{2}\right] B^{2} \\
& =\frac{1}{N\left(N^{2}+1\right)} 72\left(N^{2}-4\right)\left(N+k_{1}\right)^{2}\left(N+k_{2}\right)^{2} B^{2} \text {, } \\
& d_{25}=\left[-\frac{2\left(N+2 k_{1}\right)\left(N^{2}-4\right)\left(N^{2}-3\right)}{N\left(N^{2}+1\right)} A_{2} A_{3}+\frac{12\left(N+k_{2}\right)\left(N^{2}-4\right)\left(N^{2}+5\right)}{N\left(N^{2}+1\right)} A_{3} A_{4}\right. \\
& +\left(\frac{2}{N}\left(N+2 k_{2}\right)\left(N^{2}-4\right)+\frac{2\left(N^{2}-4\right)\left(N^{2}-3\right)}{N^{2}+1}\right) A_{3}^{2}+\frac{2\left(N^{2}-4\right)\left(N+2 k_{1}\right)}{N} A_{2}^{2} \\
& \left.-\frac{16\left(N^{2}-4\right)}{N^{2}+1} A_{3}^{2}\right] B^{2} \\
& =\frac{72}{N\left(N^{2}+1\right)}\left(N^{2}-4\right)\left(N+k_{1}\right)^{2}\left(N+2 k_{1}\right)\left(N+k_{2}\right)^{2}\left(N+k_{1}+k_{2}\right)\left[-3 k_{1}+k_{2}-N\right. \\
& \left.+k_{1} N^{2}+k_{2} N^{2}+N^{3}\right] B^{2} \text {, }
\end{aligned}
$$

where $B\left(k_{1}, k_{2}, N\right)$ is given by (I.2). The large $N$ limit (3.19) can be obtained as before

$$
\begin{aligned}
d_{2} & =\frac{2(-2+\lambda) \lambda}{3 N^{3}(2+\lambda)}, \quad d_{3}=\frac{\lambda^{2}}{N^{3}(2+\lambda)}, \quad d_{4}=\frac{2 \lambda^{2}}{3 N^{3}(-2+\lambda)(2+\lambda)}, \\
d_{6} & =\frac{(-2+\lambda)(-1+\lambda)}{2 N^{3}(2+\lambda)}, \quad d_{8}=-\frac{2 \lambda^{2}}{N^{3}(-2+\lambda)(2+\lambda)}, \quad d_{10}=-\frac{\lambda^{4}}{2 N^{4}(-2+\lambda)(2+\lambda)}, \\
d_{12} & =\frac{8(-2+\lambda) \lambda}{N^{4}(2+\lambda)}, \quad d_{13}=\frac{4 \lambda^{2}}{N^{4}(2+\lambda)}, \quad d_{14}=\frac{8 \lambda^{3}}{N^{4}(-2+\lambda)(2+\lambda)}, \\
d_{16} & =\frac{-1+\lambda}{2 N}, \quad d_{17}=\frac{\lambda(22+\lambda)}{6 N(2+\lambda)}, \quad d_{18}=-\frac{\lambda^{2}}{2 N^{2}},
\end{aligned}
$$




$$
\begin{aligned}
& d_{20}=\frac{\lambda^{2}}{N(2+\lambda)}, \quad d_{22}=\frac{\lambda\left(-12-12 \lambda+\lambda^{2}\right)}{6 N(-2+\lambda)(2+\lambda)}, \quad d_{23}=\frac{8 \lambda^{2}}{N^{4}(2+\lambda)}, \\
& d_{24}=-\frac{4 \lambda}{N^{2}(2+\lambda)}, \quad d_{25}=\frac{4 \lambda^{2}}{N^{2}(-2+\lambda)(2+\lambda)} .
\end{aligned}
$$

Note that there are the $\frac{1}{N^{4}}$ dependence in $d_{10}, d_{12}, d_{13}, d_{14}, d_{23}$ and $\frac{1}{N}$ dependence in $d_{16}, d_{17}, d_{20}, d_{22}$ and $\frac{1}{N^{2}}$ dependence in $d_{18}, d_{24}, d_{25}$. The $\widetilde{\Lambda}(w)$ is defined in previous Appendix $G$ when we discuss the operator product expansion (G.8). The field $J^{a} J^{b} K^{a} K^{b}(w)$ appears on the right hand side of (I.3) and also on the left side of (I.3) through $\widetilde{\Lambda}$ together with the relation (G.7).

We would like to see the explicit form for the $\widetilde{R}^{(4)}(z)$. Let us present the relevant $\frac{1}{(z-w)^{2}}$ terms in the operator product expansions in details and explain how we get ([.3]):

$$
\begin{aligned}
& \left.Q(z) Q(w)\right|_{\frac{1}{(z-w)^{2}}}=-9\left(N+k_{1}\right) Q^{a} Q^{a}-\frac{9}{N}\left(N+k_{1}\right)\left(N+2 k_{1}\right)\left(N^{2}-4\right) J^{a} \partial^{2} J^{a}, \\
& \left.Q(z) Q^{a} K^{a}(w)\right|_{\frac{1}{(z-w)^{2}}}=-3\left(N+k_{1}\right)\left(2 d^{a b c} J^{a} Q^{b} K^{c}-\frac{1}{N}\left(N+2 k_{1}\right)\left(N^{2}-4\right) \partial^{2} J^{a} K^{a}\right) \text {, } \\
& \left.Q(z) J^{a} R^{a}(w)\right|_{\frac{1}{(z-w)^{2}}}=-3\left(N+k_{1}\right) Q^{a} R^{a}, \\
& \left.Q^{a} K^{a}(z) Q(w)\right|_{\frac{1}{(z-w)^{2}}}=-3\left(N+k_{1}\right)\left(2 d^{a b c} J^{a} Q^{b} K^{c}-\frac{1}{N}\left(N+2 k_{1}\right)\left(N^{2}-4\right) J^{a} \partial^{2} K^{a}\right), \\
& \left.Q^{a} K^{a}(z) Q^{b} K^{b}(w)\right|_{\frac{1}{(z-w)^{2}}}=d^{a b c d}\left[\frac{2}{3} N J^{a} J^{b} J^{c} K^{d}-\left(N+2 k_{1}\right) J^{a} J^{b} K^{c} K^{d}\right] \\
& +\quad d^{a b e} d^{c d e}\left[-k_{2} J^{a} J^{b} J^{c} J^{d}+2 k_{1} J^{a} J^{b} K^{c} K^{d}\right] \\
& +\frac{8\left(N^{2}-4\right)}{N^{2}+1} J^{a} J^{a} J^{b} K^{b}-\frac{4\left(N^{2}-4\right)\left(N+2 k_{1}\right)}{N\left(N^{2}+1\right)} J^{a} J^{a} K^{b} K^{b} \\
& +\frac{2 k_{2}}{N}\left(N^{2}-4\right)\left(N+2 k_{1}\right) \partial^{2} J^{a} J^{a} \\
& +\left[-2\left(N+2 k_{1}\right)\left(N^{2}-4\right)-\frac{4 N\left(N^{2}-4\right)\left(N^{2}+3\right)}{3\left(N^{2}+1\right)}\right] \partial^{2} J^{a} K^{a} \\
& +\frac{1}{N}\left(N^{2}-4\right) k_{1}\left(N+2 k_{1}\right) \partial^{2} K^{a} K^{a} \\
& -\frac{\left(N+2 k_{1}\right)\left(N^{2}-4\right)\left(N^{2}-3\right)}{N^{2}+1} \partial J^{a} \partial K^{a} \\
& -2\left(N+2 k_{1}\right)\left(N^{2}-4\right) J^{a} \partial^{2} K^{a} \\
& -\frac{8\left(N^{2}-4\right)\left(N+2 k_{1}\right)}{N\left(N^{2}+1\right)} J^{a} J^{b} K^{a} K^{b} \\
& +\frac{2\left(N^{2}-4\right)\left(N+2 k_{1}\right)}{N} f^{a b c} J^{a} K^{b} \partial K^{c} \\
& +\left[\frac{2}{N}\left(N+2 k_{1}\right)\left(N^{2}-4\right)+\frac{2\left(N^{2}-4\right)\left(N^{2}+5\right)}{N^{2}+1}\right] f^{a b c} J^{a} \partial J^{b} K^{c}, \\
& \left.Q^{a} K^{a}(z) J^{b} R^{b}(w)\right|_{\frac{1}{(z-w)^{2}}}=-\left(N+2 k_{2}\right) d^{a b c} J^{a} Q^{b} K^{c}+2 N Q^{a} R^{a}
\end{aligned}
$$




$$
\begin{aligned}
&\left(N+2 k_{1}\right) d^{a b c} J^{a} K^{b} R^{c} \\
&+ \frac{1}{N}\left(N+2 k_{1}\right)\left(N+2 k_{2}\right)\left(N^{2}-4\right) \partial^{2} J^{a} K^{a}, \\
&\left.Q^{a} K^{a}(z) R(w)\right|_{\frac{1}{(z-w)^{2}}}=-3\left(N+k_{2}\right) Q^{a} R^{a}, \\
&\left.J^{a} R^{a}(z) Q(w)\right|_{\frac{1}{(z-w)^{2}}}=\left[\left.Q^{a} K^{a}(z) R(w)\right|_{\frac{1}{(z-w)^{2}}}\right]_{J^{c} \leftrightarrow K^{c}, k_{1} \leftrightarrow k_{2}}, \\
&\left.J^{a} R^{a}(z) Q^{b} K^{b}(w)\right|_{\frac{1}{(z-w)^{2}}}=\left[\left.Q^{a} K^{a}(z) J^{b} R^{b}(w)\right|_{\frac{1}{(z-w)^{2}}}\right]_{J^{c} \leftrightarrow K^{c}, k_{1} \leftrightarrow k_{2}}, \\
&\left.J^{a} R^{a}(z) J^{a} R^{a}(w)\right|_{\frac{1}{(z-w)^{2}}}=\left[\left.Q^{a} K^{a}(z) Q^{b} K^{b}(w)\right|_{\frac{1}{(z-w)^{2}}}\right]_{J^{c} \leftrightarrow K^{c}, k_{1} \leftrightarrow k_{2}}, \\
&\left.J^{a} R^{a}(z) R(w)\right|_{\frac{1}{(z-w)^{2}}}=\left[\left.Q^{a} K^{a}(z) Q(w)\right|_{\frac{1}{(z-w)^{2}}}\right]_{J^{c} \leftrightarrow K^{c}, k_{1} \leftrightarrow k_{2}}, \\
&\left.R(z) Q^{a} K^{a}(w)\right|_{\frac{1}{(z-w)^{2}}}=\left[\left.Q(z) J^{a} R^{a}(w)\right|_{\frac{1}{(z-w)^{2}}}\right]_{J^{c} \leftrightarrow K^{c}, k_{1} \leftrightarrow k_{2}}, \\
&\left.R(z) J^{a} R^{a}(w)\right|_{\frac{1}{(z-w)^{2}}}=\left[\left.Q(z) Q^{a} K^{a}(w)\right|_{\frac{1}{(z-w)^{2}}}\right]_{J^{c} \leftrightarrow K^{c}, k_{1} \leftrightarrow k_{2}}, \\
&\left.R(z) R(w)\right|_{\frac{1}{(z-w)^{2}}}=\left[\left.Q(z) Q(w)\right|_{\left.\frac{1}{(z-w)^{2}}\right]_{J^{c} \leftrightarrow K^{c}, k_{1} \leftrightarrow k_{2}},},\right.
\end{aligned}
$$

where $R(w) \equiv d^{a b c} K^{a} K^{b} K^{c}(w)$ corresponding to (2.6). The right hand side of the first equation of (I.6) corresponds to the spin 4 field $R_{4}(w)$ in (3.2) of [4] up to an overall constant. Here the last seven operator product expansions (I.6) can be obtained from the first seven operator product expansions using the properties $J^{a} \leftrightarrow K^{a}$ and $k_{1} \leftrightarrow k_{2}$.

At first sight, the spin 4 field (I.3) looks different from (3.16), but the independent field contents are the same except the one field $J^{a} J^{b} K^{a} K^{b}(z)$. Let us look at the structures in details. First of all, one has the following identities

$$
\begin{aligned}
f^{a b c} J^{a} \partial J^{b} K^{c}(w) & =\frac{N\left(N^{2}+1\right)}{\left(N^{2}-4\right)\left(N^{2}-3\right)}\left[-d^{a b e} d^{c d e} J^{a} J^{b} J^{c} K^{d}(w)+\frac{1}{3} d^{a b c d} J^{a} J^{b} J^{c} K^{d}(w)\right. \\
& \left.+\frac{4\left(N^{2}-4\right)}{N\left(N^{2}+1\right)} J^{a} J^{a} J^{b} K^{b}(w)+\frac{\left(N^{2}-4\right)\left(N^{2}-3\right)}{3\left(N^{2}+1\right)} \partial^{2} J^{a} K^{a}(w)\right], \\
f^{a b c} J^{a} K^{b} \partial K^{c}(w) & =\frac{N\left(N^{2}+1\right)}{\left(N^{2}-4\right)\left(N^{2}-3\right)}\left[d^{a b e} d^{c d e} J^{a} K^{b} K^{c} K^{d}(w)-\frac{1}{3} d^{a b c d} J^{a} K^{b} K^{c} K^{d}(w)\right. \\
& \left.-\frac{4\left(N^{2}-4\right)}{N\left(N^{2}+1\right)} J^{a} K^{a} K^{b} K^{b}(w)+\frac{2\left(N^{2}-4\right)\left(N^{2}-3\right)}{3\left(N^{2}+1\right)} J^{a} \partial^{2} K^{a}(w)\right], \\
\partial J^{a} \partial J^{a}(w) & =\frac{\left(N^{2}+1\right)}{3\left(N^{2}-4\right)\left(N^{2}-3\right)}\left[-d^{a b c d} J^{a} J^{b} J^{c} J^{d}(w)+3 d^{a b e} d^{c d e} J^{a} J^{b} J^{c} J^{d}(w)\right. \\
& \left.-\frac{12\left(N^{2}-4\right)}{N\left(N^{2}+1\right)} J^{a} J^{a} J^{b} J^{b}(w)+\frac{2\left(N^{2}-4\right)\left(N^{2}-3\right)}{\left(N^{2}+1\right)} \partial^{2} J^{a} J^{a}(w)\right], \\
\partial K^{a} \partial K^{a}(w) & =\frac{\left(N^{2}+1\right)}{3\left(N^{2}-4\right)\left(N^{2}-3\right)}\left[-d^{a b c d} K^{a} K^{b} K^{c} K^{d}(w)+3 d^{a b e} d^{c d e} K^{a} K^{b} K^{c} K^{d}(w)\right.
\end{aligned}
$$




$$
\left.-\frac{12\left(N^{2}-4\right)}{N\left(N^{2}+1\right)} K^{a} K^{a} K^{b} K^{b}(w)+\frac{2\left(N^{2}-4\right)\left(N^{2}-3\right)}{\left(N^{2}+1\right)} \partial^{2} K^{a} K^{a}(w)\right] .
$$

The first and second relations of (I.7) can be obtained from the first and third relations of (G.1), respectively. These two fields appear in (I.6). We want to reexpress (I.3) in terms of the fields in (3.16). Then, the $d_{24}$ term in (1.3) can be absorbed into $d_{2}, d_{12}, d_{17}$ and a new term $d^{a b e} d^{c d e} J^{a} J^{b} J^{c} K^{d}(w)$ corresponding to $c_{7}$ term in (3.16). Similarly, the $d_{25}$ term in (II.3) can be absorbed into $d_{4}, d_{14}, d_{22}$ and a new term $d^{a b e} d^{c d e} J^{a} K^{b} K^{c} K^{d}(w)$ corresponding to $c_{9}$ term in (3.16). The third and fourth relations of (I.7) can be obtained from the relation (3.17). The reason why we use these equations comes from the discussion in the subsection 3.2 where we keep $c_{19}=0=c_{21}$. Since the left hand side of (I.3) contains $\widetilde{\Lambda}(w)$ and $\partial^{2} \widetilde{T}(w)$, one sees $\partial J^{a} \partial J^{a}(w)$ term and $\partial K^{a} \partial K^{a}(w)$ term in the field $\widetilde{R}^{(4)}(w)$. So we have to use the third and fourth equations of (II.7) and reexpress them in terms of other independent fields.

Moreover, note that there are the following identities

$$
\begin{aligned}
d^{a b c} J^{a} Q^{b} K^{c}(w) & =\frac{1}{3} d^{a b c d} J^{a} J^{b} J^{c} K^{d}(w)+\frac{4\left(N^{2}-4\right)}{N\left(N^{2}+1\right)} J^{a} J^{a} J^{b} K^{b}(w) \\
& +\frac{\left(N^{2}-4\right)\left(N^{2}+5\right)}{N\left(N^{2}+1\right)} f^{a b c} J^{a} \partial J^{b} K^{c}(w)-\frac{2\left(N^{2}-4\right)\left(N^{2}+3\right)}{3\left(N^{2}+1\right)} \partial^{2} J^{a} K^{a}(w), \\
d^{a b c} J^{a} K^{b} R^{c}(w) & =\frac{1}{3} d^{a b c d} J^{a} K^{b} K^{c} K^{d}(w)+\frac{4\left(N^{2}-4\right)}{N\left(N^{2}+1\right)} J^{a} K^{b} K^{b} K^{a}(w) \\
& +\frac{\left(N^{2}-4\right)\left(N^{2}+5\right)}{N\left(N^{2}+1\right)} f^{a b c} J^{a} K^{b} \partial K^{c}(w)-\frac{2\left(N^{2}-4\right)\left(N^{2}+3\right)}{3\left(N^{2}+1\right)} J^{a} \partial^{2} K^{a}(w) .
\end{aligned}
$$

Using the first equation of ([1.8), for example, one simplifies the $\frac{1}{(z-w)^{2}}$ term in the operator product expansion $Q(z) Q^{a} K^{a}(w)$ in (1.6). See also the relation (E.24). Similarly, by the second equation of (I.8), one can simplify the $\frac{1}{(z-w)^{2}}$ term in the operator product expansion


using the equations (I.7). By plugging the expressions (I.7) and (I.8) into (I.6) , one has the independent terms as in the relation (3.16) ) as well as the term $J^{a} J^{b} K^{a} K^{b}(z)$.

All the coefficients turn out to be (I.4). It turns out that the coset spin 4 field $\widetilde{R}^{(4)}(w)$ is given by the previous one $\widetilde{W}^{(4)}(w)$ (3.39) by realizing that the undetermined two coefficients $c_{1}$ and $c_{23}$ become

$$
\begin{aligned}
c_{1} & =-\frac{k_{2}\left(N^{2}+1\right)}{2\left(N^{2}-4\right)\left(N^{2}-3\right)\left(N+k_{1}\right)\left(N+k_{1}+k_{2}\right) d\left(k_{1}, k_{2}, N\right)}\left[-3 k_{1}^{2} k_{2}-2 k_{1}^{2} N-8 k_{1} k_{2} N\right. \\
& \left.-2 k_{2}^{2} N-4 k_{1} N^{2}-4 k_{2} N^{2}+k_{1}^{2} k_{2} N^{2}+k_{1} k_{2}^{2} N^{2}-2 N^{3}+2 k_{1} k_{2} N^{3}-3 k_{1} k_{2}^{2}\right], \\
c_{23} & =\frac{-8}{\left(N+k_{1}+k_{2}\right)\left(3 N+2 k_{1}+2 k_{2}\right)\left(N^{2}+1\right) d\left(k_{1}, k_{2}, N\right)}\left[-9 k_{1}^{2} k_{2}-9 k_{1} k_{2}^{2}-14 k_{1}^{2} N\right.
\end{aligned}
$$




$$
\begin{aligned}
& -28 k_{1} k_{2} N-14 k_{2}^{2} N-24 k_{1} N^{2}-24 k_{2} N^{2}+3 k_{1}^{2} k_{2} N^{2}+3 k_{1} k_{2}^{2} N^{2}-10 N^{3}+8 k_{1}^{2} N^{3} \\
& \left.+\quad 18 k_{1} k_{2} N^{3}+8 k_{2}^{2} N^{3}+20 k_{1} N^{4}+20 k_{2} N^{4}+12 N^{5}\right]
\end{aligned}
$$

where the function $d\left(k_{1}, k_{2}, N\right)$ is introduced as follows:

$$
\begin{aligned}
d\left(k_{1}, k_{2}, N\right) & \equiv 17 k_{1}^{2} k_{2}+17 k_{1} k_{2}^{2}+22 k_{1}^{2} N+56 k_{1} k_{2} N+22 k_{2}^{2} N+44 k_{1} N^{2}+44 k_{2} N^{2} \\
& +5 k_{1}^{2} k_{2} N^{2}+5 k_{1} k_{2}^{2} N^{2}+22 N^{3}+10 k_{1} k_{2} N^{3}
\end{aligned}
$$

That is, the spin 4 field $\widetilde{R}^{(4)}(w)$ is nothing but the field $\widetilde{W}^{(4)}(w)$,

$$
\widetilde{R}^{(4)}(w)=\widetilde{W}^{(4)}(w), \quad \text { with }(\underline{I .9}) \text { and }(\underline{I .10}) \text {. }
$$

The $c_{1}$ term comes from the $\partial^{2} \widetilde{T}(w)$ in (1.3) together with (1.7). The $c_{23}$ term comes from $\widetilde{T}^{2}(w)$ term and $J^{a} J^{b} K^{a} K^{b}(w)$ term characterized by $d_{23}$ in (I.3). One can easily obtain the large $N$ 't Hooft limit (3.19) for these coefficients and they are given by

$$
c_{1}(N, \lambda)=\frac{-1+\lambda}{10 N^{3}}, \quad c_{23}(N, \lambda)=\frac{32 \lambda^{2}}{5 N^{3}(-1+\lambda)(1+\lambda)},
$$

where one uses the relations (I.5) . It is easy to check that the operator product expansion between $J^{\prime a}(z)$ and $\widetilde{R}^{(4)}(w)$ does not have any singular terms. That is, $J^{\prime a}(z) \widetilde{R}^{(4)}(w)=0$. This implies that the operator product expansion between $J^{\prime a}(z)$ and the right hand side of (I.3) gives no singular terms (the operator product expansion $J^{\prime a}(z) \widetilde{T}(w)$ vanishes). Similarly, one can check that the operator product expansion between $\hat{T}(z)$ and $\widetilde{R}^{(4)}(w)$ does not have any higher order singular terms $\frac{1}{(z-w)^{n}}, n>2$ by using the fact that $T^{\prime}(z) \widetilde{R}^{(4)}(w)=0$.

The large $N$ behavior for the zero mode of $\widetilde{R}^{(4)}(z)$ is described at the end of the subsection

3.4 and due to the fact that this spin- 4 field is the field $\widetilde{W}^{(4)}(z)$, one has also the relation (3.44).

\section{References}

[1] M. R. Gaberdiel and R. Gopakumar, "An $A d S_{3}$ Dual for Minimal Model CFTs," Phys. Rev. D 83, 066007 (2011) arXiv:1011.2986 [hep-th]].

[2] P. Goddard, A. Kent and D. I. Olive, "Virasoro Algebras and Coset Space Models," Phys. Lett. B 152, 88 (1985).

[3] P. Goddard, A. Kent and D. I. Olive, "Unitary Representations of the Virasoro and Supervirasoro Algebras," Commun. Math. Phys. 103, 105 (1986). 
[4] F. A. Bais, P. Bouwknegt, M. Surridge and K. Schoutens, "Extensions of the Virasoro Algebra Constructed from Kac-Moody Algebras Using Higher Order Casimir Invariants," Nucl. Phys. B 304, 348 (1988).

[5] F. A. Bais, P. Bouwknegt, M. Surridge and K. Schoutens, "Coset Construction for Extended Virasoro Algebras," Nucl. Phys. B 304, 371 (1988).

[6] S. F. Prokushkin and M. A. Vasiliev, "Higher spin gauge interactions for massive matter fields in 3-D AdS space-time," Nucl. Phys. B 545, 385 (1999) arXiv:hep-th/9806236.

[7] S. Prokushkin and M. A. Vasiliev, "3-d higher spin gauge theories with matter," arXiv:hep-th/9812242.

[8] M. A. Vasiliev, "Higher spin gauge theories: Star product and AdS space," arXiv:hep-th/9910096.

[9] V. A. Fateev and S. L. Lukyanov, "The Models of Two-Dimensional Conformal Quantum Field Theory with Z(n) Symmetry," Int. J. Mod. Phys. A 3, 507 (1988).

[10] P. Bouwknegt and K. Schoutens, "W symmetry in conformal field theory," Phys. Rept. 223, 183 (1993) arXiv:hep-th/9210010.

[11] M. R. Gaberdiel, R. Gopakumar, T. Hartman and S. Raju, "Partition Functions of Holographic Minimal Models," JHEP 1108, 077 (2011) [arXiv:1106.1897 [hep-th]].

[12] P. Di Francesco, C. Itzykson and J. B. Zuber, "Classical W algebras," Commun. Math. Phys. 140, 543 (1991).

[13] V. G. Drinfeld and V. V. Sokolov, "Lie algebras and equations of Korteweg-de Vries type," J. Sov. Math. 30, 1975 (1984).

[14] C. M. Chang and X. Yin, "Higher Spin Gravity with Matter in $A d S_{3}$ and Its CFT Dual," arXiv:1106.2580 [hep-th].

[15] G. M. T. Watts, "Determinant formulae for extended algebra in two-dimensional conformal field theory," Nucl. Phys. B 326, 648 (1989)(Erratum-ibid.B336:720,1990).

[16] M. R. Gaberdiel and T. Hartman, "Symmetries of Holographic Minimal Models," JHEP 1105, 031 (2011) [arXiv:1101.2910 [hep-th]].

[17] K. Thielemans, "A Mathematica package for computing operator product expansions," Int. J. Mod. Phys. C 2, 787 (1991). 
[18] A. Campoleoni, "Higher Spins in $\mathrm{D}=2+1$, , arXiv:1110.5841 [hep-th].

[19] B. Chen and J. Long, "High Spin Topologically Massive Gravity," arXiv:1110.5113 [hepth].

[20] S. Giombi, S. Minwalla, S. Prakash, S. P. Trivedi, S. R. Wadia and X. Yin, "Chern-Simons Theory with Vector Fermion Matter," arXiv:1110.4386 [hep-th].

[21] A. Castro, E. Hijano, A. Lepage-Jutier and A. Maloney, "Black Holes and Singularity Resolution in Higher Spin Gravity," arXiv:1110.4117 [hep-th].

[22] S. H. Shenker and X. Yin, "Vector Models in the Singlet Sector at Finite Temperature," arXiv:1109.3519 [hep-th].

[23] M. Vasiliev, "Cubic Vertices for Symmetric Higher-Spin Gauge Fields in $(A) d S_{d}$," arXiv:1108.5921 [hep-th].

[24] K. Papadodimas and S. Raju, "Correlation Functions in Holographic Minimal Models," arXiv:1108.3077 [hep-th].

[25] P. Kraus and E. Perlmutter, "Partition functions of higher spin black holes and their CFT duals," arXiv:1108.2567 [hep-th].

[26] A. Castro, T. Hartman and A. Maloney, "The Gravitational Exclusion Principle and Null States in Anti-de Sitter Space," Class. Quant. Grav. 28, 195012 (2011) arXiv:1107.5098 [hep-th]].

[27] A. Bagchi, S. Lal, A. Saha and B. Sahoo, "One loop partition function for Topologically Massive Higher Spin Gravity," arXiv:1107.2063 [hep-th].

[28] A. Bagchi, S. Lal, A. Saha and B. Sahoo, "Topologically Massive Higher Spin Gravity," arXiv:1107.0915 [hep-th].

[29] A. Campoleoni, S. Fredenhagen and S. Pfenninger, "Asymptotic W-symmetries in threedimensional higher-spin gauge theories," arXiv:1107.0290 [hep-th].

[30] B. Chen, J. Long and J. b. Wu, "Spin-3 Topological Massive Gravity," arXiv:1106.5141 [hep-th].

[31] M. Ammon, M. Gutperle, P. Kraus and E. Perlmutter, "Spacetime Geometry in Higher Spin Gravity," arXiv:1106.4788 [hep-th]. 
[32] A. Jevicki, K. Jin and Q. Ye, "Collective Dipole Model of AdS/CFT and Higher Spin Gravity," arXiv:1106.3983 [hep-th].

[33] M. Gutperle and P. Kraus, "Higher Spin Black Holes," JHEP 1105, 022 (2011) arXiv:1103.4304 [hep-th]].

[34] R. Gopakumar, R. K. Gupta and S. Lal, "The Heat Kernel on AdS," arXiv:1103.3627 [hep-th].

[35] X. Bekaert, E. Joung and J. Mourad, "Effective action in a higher-spin background," JHEP 1102, 048 (2011) [arXiv:1012.2103 [hep-th]].

[36] A. Castro, A. Lepage-Jutier and A. Maloney, "Higher Spin Theories in $A d S_{3}$ and a Gravitational Exclusion Principle," JHEP 1101, 142 (2011) [arXiv:1012.0598 [hep-th]].

[37] K. Alkalaev, "FV-type action for AdS(5) mixed-symmetry fields," JHEP 1103, 031 (2011) arXiv:1011.6109 [hep-th]].

[38] M. R. Douglas, L. Mazzucato and S. S. Razamat, "Holographic dual of free field theory," Phys. Rev. D 83, 071701 (2011) arXiv:1011.4926 [hep-th]].

[39] H. T. Ozer, "On the construction of $\mathrm{W}(\mathrm{N})$ algebras in the form of $\mathrm{A}(\mathrm{N}-1)$ Casimir algebras," Mod. Phys. Lett. A 11, 1139 (1996) arXiv:hep-th/9609120.

[40] K. Schoutens, "Yangian symmetry in conformal field theory," Phys. Lett. B 331, 335 (1994) arXiv:hep-th/9401154.

[41] J. A. De Azcarraga and J. C. Perez Bueno, "Higher-order simple Lie algebras," Commun. Math. Phys. 184, 669 (1997) arXiv:hep-th/9605213.

[42] J. A. de Azcarraga, A. J. Macfarlane, A. J. Mountain and J. C. Perez Bueno, "Invariant tensors for simple groups," Nucl. Phys. B 510, 657 (1998) arXiv:physics/9706006.

[43] J. Thierry-Mieg, "Generalization of the Sugawara construction," DAMTP-87-29, Nov 1987, Lecture given at Cargese School on Nonperturbative Quantum Field Theory, Cargese, France, Jul 16-30, 1987. Published in Cargese Summer Inst.1987:0567-576.

[44] R. Blumenhagen, M. Flohr, A. Kliem, W. Nahm, A. Recknagel and R. Varnhagen, "W algebras with two and three generators," Nucl. Phys. B 361, 255 (1991).

[45] H. G. Kausch and G. M. T. Watts, "A Study of W algebras using Jacobi identities," Nucl. Phys. B 354, 740 (1991). 
[46] P. Bouwknegt, "Extended conformal algebras," Phys. Lett. B 207, 295 (1988).

[47] K. J. Hamada and M. Takao, "Spin 4 current algebra," Phys. Lett. 209B, 247 (1988) [Erratum-ibid. B 213, 564 (1988)].

[48] D. H. Zhang, "Spin 4 extended conformal algebra," Phys. Lett. B 232, 323 (1989).

[49] C. Ahn, K. Schoutens, A. Sevrin, "The full structure of the super W(3) algebra," Int. J. Mod. Phys. A6, 3467-3488 (1991).

[50] C. Ahn, "The Large N 't Hooft Limit of Coset Minimal Models," arXiv:1106.0351 [hepth].

[51] M. R. Gaberdiel and C. Vollenweider, "Minimal Model Holography for $\mathrm{SO}(2 \mathrm{~N})$," arXiv:1106.2634 [hep-th].

[52] C. Ahn, "Explicit construction of spin 4 Casimir operator in the coset model $\mathrm{SO}(5)-1 \mathrm{x}$ SO(5)-m / SO(5)-(1+m)," J. Phys. A A27, 231-238 (1994). hep-th/9209001.

[53] P. Bowcock and G. M. T. Watts, "On the classification of quantum W algebras," Nucl. Phys. B 379, 63 (1992) arXiv:hep-th/9111062. 